\title{
Cu-Catalyzed Carbonylative Silylation of Alkyl Halides: Efficient Access to Acylsilanes
}

\author{
Li-Jie Cheng and Neal P. Mankad* \\ Department of Chemistry, University of Illinois at Chicago \\ 845 W. Taylor St., Chicago, IL 60607, USA. \\ ${ }^{\star}$ Email: npm@uic.edu
}

\section{Supporting Information}

\section{Table of Contents}

1. General Information $\quad S 2$

2. General Procedure for Optimizations $\quad$ S2

3. General Procedure for Synthesis of Acylsilanes S5

4. Preparation of Alkyl Halides Substrates $\quad S 16$

5. Use of Other Bpin-SiR Reagents $\quad S 18$

6. One-pot Synthesis of $\alpha$-Hydroxylsilanes $\quad S 19$

7. Mechanistic Studies $\quad$ S20

8. ${ }^{1} \mathrm{H},{ }^{13 \mathrm{C}}$ NMR Spectra $\quad$ S24

9. References $\quad$ S54 


\section{General Information}

General Procedures. Reactions requiring anhydrous conditions were conducted in a $\mathrm{N}_{2}$-filled glovebox or using standard Schlenk line techniques. Reactions at greater than atmospheric pressure were conducted in a Parr 4621 General Purpose Pressure Reactor. A fitted, aluminum insert was custom-made for the Parr reactor that could hold up to eight 22-mL scintillation vials for running reactions in parallel. Thin layer chromatography (TLC) was conducted with E. Merck silica gel 60 F254 pre-coated plates (0.25 mm) and visualised by UV light ( $254 \mathrm{~nm}$ ). Purification of compounds was achieved by column chromatography using Merck Flash Silica Gel 60 (230-400 mesh). Organic solutions were concentrated under reduced pressure using a rotary evaporator.

Materials. Deuterated solvents were purchased from Cambridge Isotope Laboratories, Inc. Common commercial reagents were purchased from Sigma-Aldrich, Fisher Scientific or VWR International Co. without further purification unless otherwise noted. Solvents were dried using a Glass Contour Solvent System built by Pure Process Technology, LLC. CO gas was purchased from Praxair at a purity of $99.99 \%$ (4.ORS research grade) and used directly from the cylinder. NaOPh was purchased from Alfa Aesar. $\mathrm{PhMe}_{2} \mathrm{Si}$-Bpin was purchased from TCl. IPrCuCl, ${ }^{1} \mathrm{SIPrCuCl},{ }^{2} \mathrm{IMesCuCl},{ }^{1}$ SIMesCuCl, ${ }^{2} \mathrm{MeIPrCuCl},{ }^{3}$ CIIPrCuCl, ${ }^{3} \mathrm{ICyCuCl},{ }^{2} \mathrm{Et}_{3} \mathrm{Si}-\mathrm{Bpin}{ }^{4}$ and $\mathrm{Ph}_{2} \mathrm{MeSi}-\mathrm{Bpin}^{5}$ were prepared according to literature procedures.

Instrumentation. Nuclear Magnetic Resonance (NMR) spectra were recorded on BRUKER AV (400 $\mathrm{MHz})$ or BRUKER AV $(500 \mathrm{MHz})$ at $298 \mathrm{~K}$. Chemical shifts ( $\delta)$ are reported in ppm with the residual solvent signal as internal standard (chloroform at 7.26 and $77.00 \mathrm{ppm}$ for ${ }^{1} \mathrm{H}$ and ${ }^{13} \mathrm{C}$ NMR spectroscopy, respectively). Abbreviations for signal coupling are as follows: $s$, singlet; $d$, doublet; $t$, triplet; q, quartet; quin., quintet; m, multiplet; br., broad. Coupling constants were taken from the spectra directly and are uncorrected. ${ }^{1} \mathrm{H}$ and ${ }^{13} \mathrm{C}$ NMR provided are taken directly using material for which the yield is quoted, without further purification, and are representative of purity. FT-IR spectra were recorded on a Thermo Nicolet iS5 FT-IR. Absorptions are given in wavenumbers $\left(\mathrm{cm}^{-1}\right)$. HRMS (ESI) were measured with a Shimadzu LCMS-IT-TOF Mass Spectrometer.

\section{General Procedure for Optimization}

In a glovebox, base and solvent $(4.0 \mathrm{~mL})$ were added to a $20-\mathrm{mL}$ vial with a $1.5 \mathrm{~cm}$ stir bar. The copper catalyst was dissolved in solvent $(0.5 \mathrm{~mL})$ and transferred to the above mixture. The mixture was stirred at room temperature for 2 min before $\mathrm{PhMe}_{2} \mathrm{Si}$-Bpin and 1-iodooctane $(0.05 \mathrm{mmol})$ were added sequentially. The vial was then placed in an aluminum rack within a Parr pressure reactor. Next, the reactor was closed and taken out of the glovebox. A CO gas cylinder with a pressure regulator was connected to the reactor. The reactor was pressurized with $\mathrm{CO}$ gas and released after $5 \mathrm{~min}$. This procedure was repeated three times, after which the reaction was stirred for $12 \mathrm{~h}$. Then, CO gas was released, and the solvent was removed under reduced pressure. The residue was diluted with $\mathrm{Et}_{2} \mathrm{O}$ and filtered through a pad of silica gel (a pipette with about $5 \mathrm{~cm}$ silica gel). The filtrate was concentrated under reduced pressure. 
The residue was dissolved in $\mathrm{CDCl}_{3}$ and $\mathrm{CH}_{2} \mathrm{Br}_{2}(0.1 \mathrm{mmol})$ was added as internal standard for ${ }^{1} \mathrm{H} \mathrm{NMR}$ analysis.

Table S1. Catalyst investigation

\begin{tabular}{|c|c|c|c|}
\hline \multirow{2}{*}{\multicolumn{2}{|c|}{ 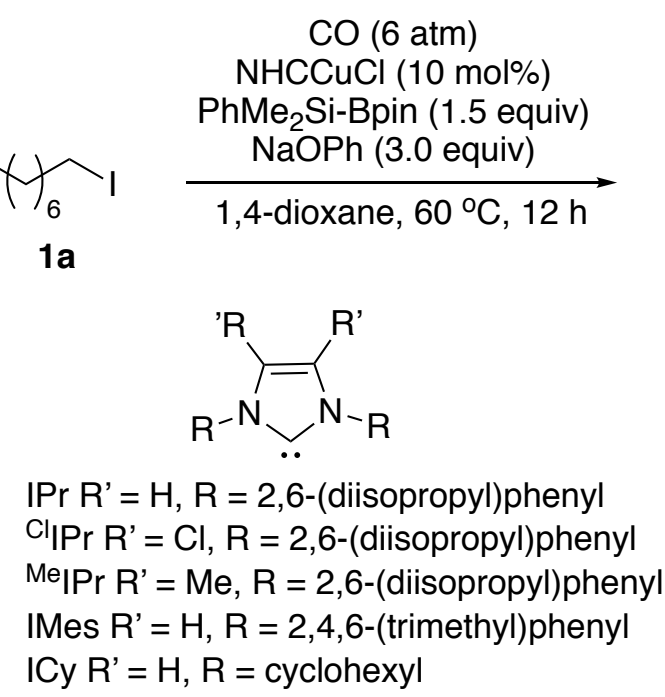 }} & & \\
\hline & & $2 a$ & $\begin{array}{l}\text { propyl)phenyl } \\
\text { trimethyl)phenyl }\end{array}$ \\
\hline Entry & Catalyst & Yield of $\mathbf{2 a}(\%)$ & Yield of $\mathbf{3 a}$ \\
\hline 1 & IPrCuCl & 93 & $<5$ \\
\hline 2 & SIPrCuCl & 52 & $<5$ \\
\hline 3 & MelPrCuCl & 30 & $<5$ \\
\hline 4 & CllPrCuCl & 83 & $<5$ \\
\hline 5 & IMesCuCl & $<5$ & 80 \\
\hline 6 & SIMesCuCl & 12 & 65 \\
\hline 7 & ICyCuCl & 0 & 99 \\
\hline 8 & No catalyst & 0 & 0 \\
\hline
\end{tabular}

Table S2. Base investigation

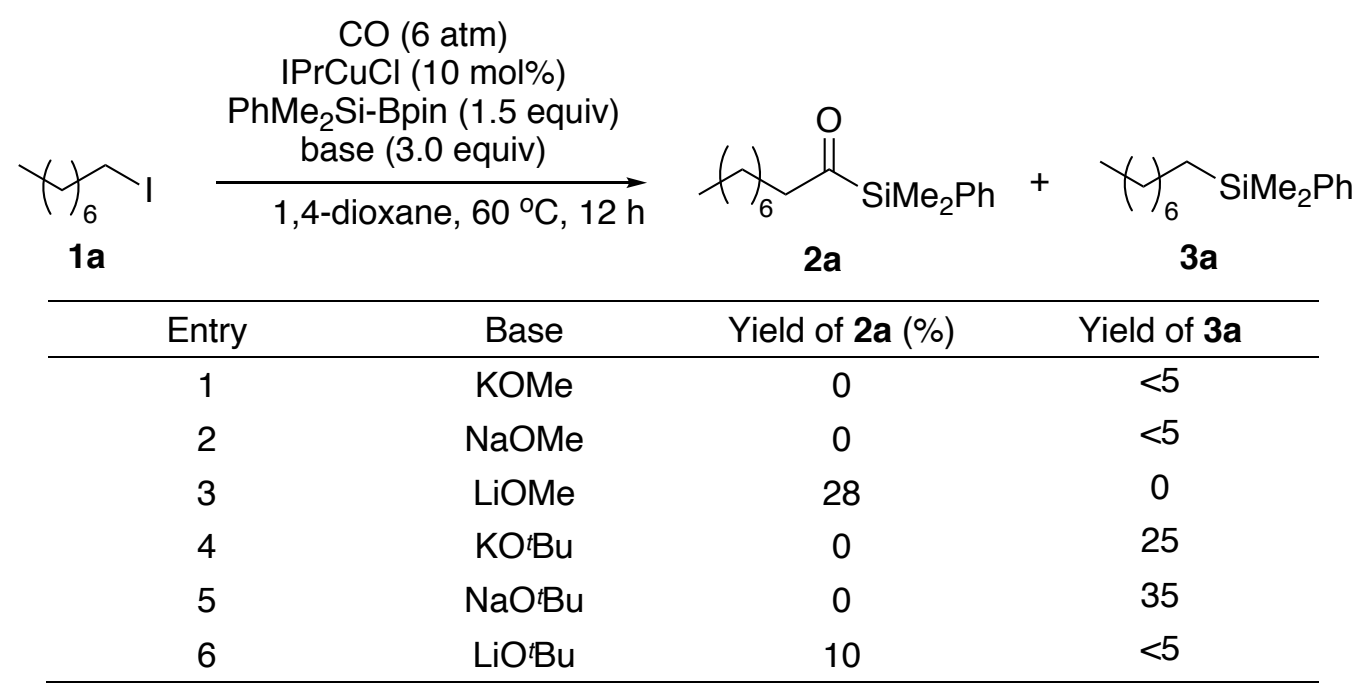




\begin{tabular}{llll}
\hline 7 & $\mathrm{NaOPh}$ & 93 & $<5$ \\
\hline
\end{tabular}

Table S3. Solvent investigation

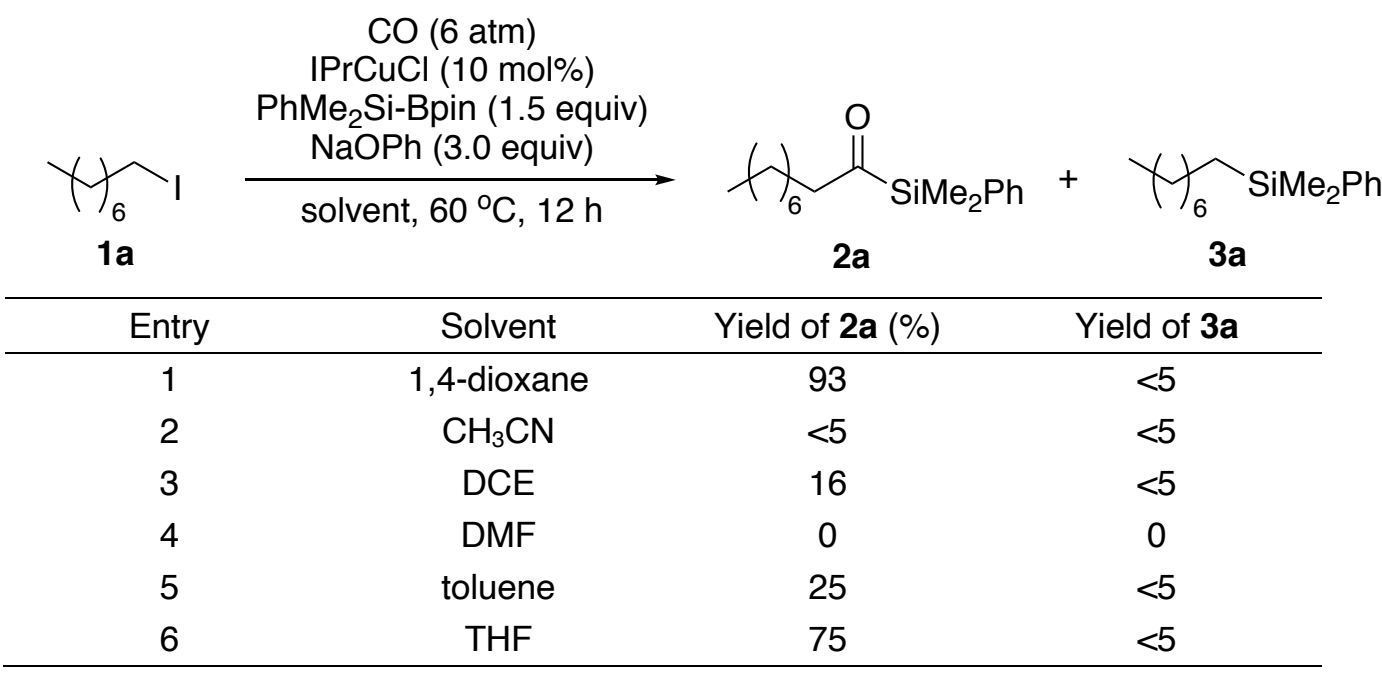

Table S4. Temperature investigation

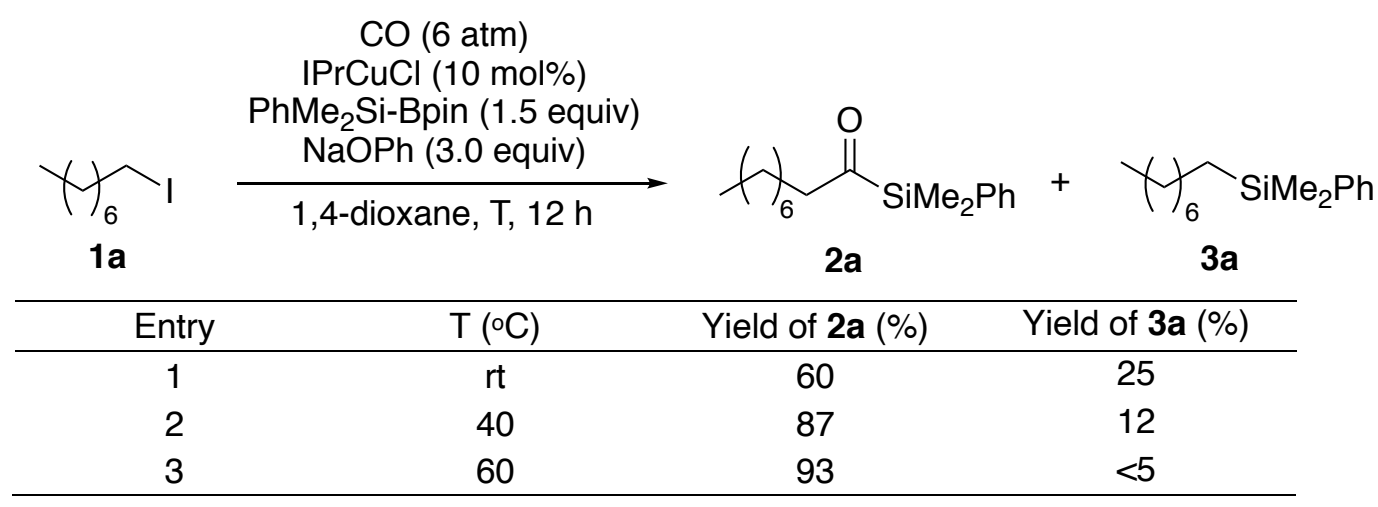

Table S5. CO pressure investigation

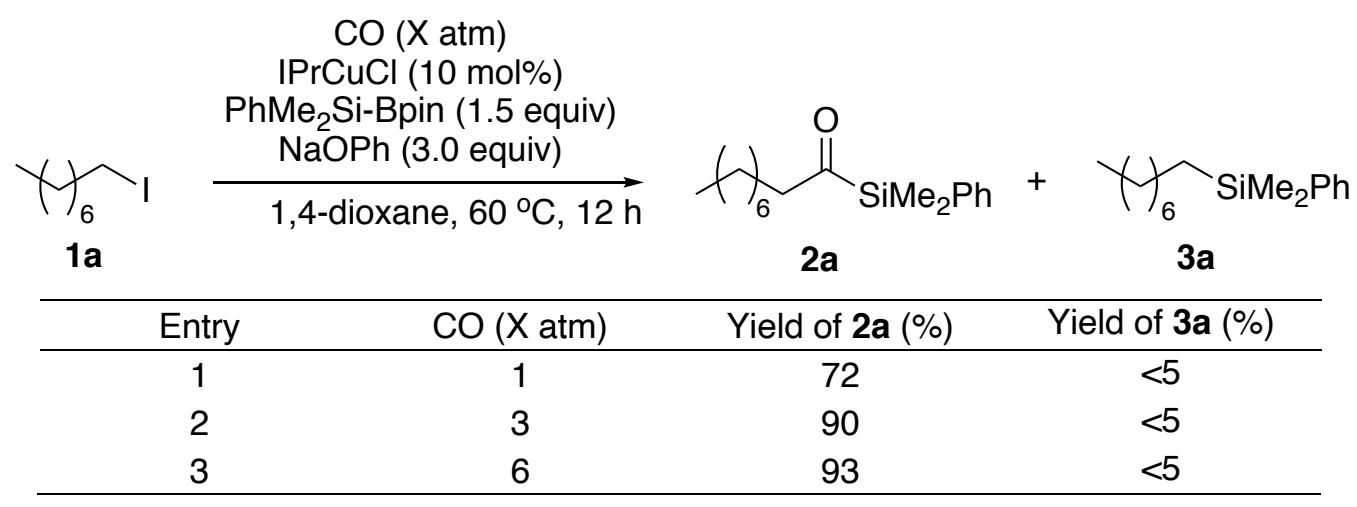

Table S6. Amount of Bpin-SiMe ${ }_{2} \mathrm{Ph}$ and base investigation 


\begin{tabular}{|c|c|c|c|c|}
\hline & $\begin{array}{c}\mathrm{CO}(6 \mathrm{~atm}) \\
\mathrm{IPrCuCl}(10 \mathrm{~mol} \%) \\
\mathrm{PhMe} \mathrm{Si}_{2} \mathrm{Sipin}(\mathrm{X} \text { equiv) } \\
\mathrm{NaOPh}(\mathrm{Y} \text { equiv) }\end{array}$ & & & \\
\hline $\mathbf{1 a}$ & 1,4-dioxane, $60^{\circ} \mathrm{C}, 12 \mathrm{~h}$ & & 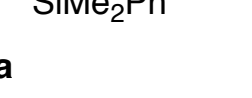 & $3 a$ \\
\hline Entry & $\mathrm{X}$ & $\mathrm{Y}$ & Yield of $\mathbf{2 a}(\%)$ & Yield of $\mathbf{3 a}(\%)$ \\
\hline 1 & 1.2 & 1.5 & 60 & $<5$ \\
\hline 2 & 1.2 & 2.0 & 78 & $<5$ \\
\hline 3 & 1.2 & 2.5 & 87 & $<5$ \\
\hline 4 & 1.2 & 3.0 & 89 & $<5$ \\
\hline 5 & 1.5 & 2.0 & 83 & $<5$ \\
\hline 6 & 1.5 & 3.0 & 93 & $<5$ \\
\hline
\end{tabular}

Table S7. Electrophiles investigation

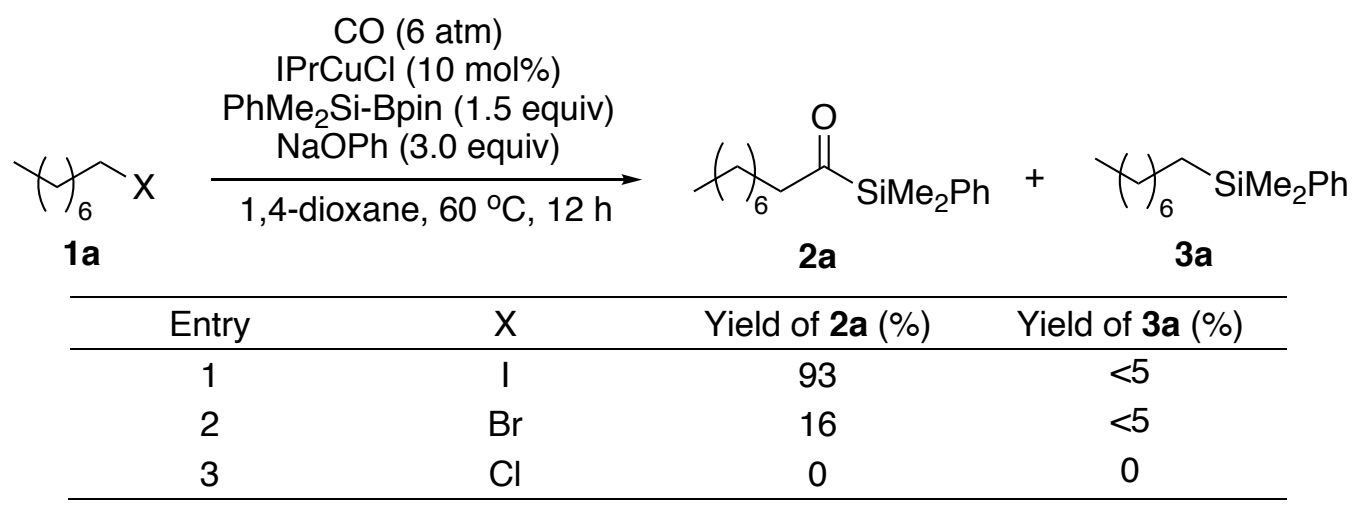

\section{General Procedure for Synthesis Acylsilanes}

In a glovebox, NaOPh (70 mg, 0.6 mmol, 3.0 equiv), IPrCuCl (9.6 mg, 0.02 mmol, $10 \mathrm{~mol} \%$ ) and 1,4dioxane $(6.0 \mathrm{~mL})$ were added to a $20-\mathrm{mL}$ vial with a $1.5 \mathrm{~cm}$ stir bar. The mixture was stirred at room temperature for 2 min before PhMe 2 Si-Bpin ( $80 \mu \mathrm{L}, 0.3 \mathrm{mmol}, 1.5$ equiv) and alkyl halide $(0.2 \mathrm{mmol})$ were added sequentially at room temperature. The vial was then placed in an aluminum rack within a Parr pressure reactor. Next, the reactor was closed and taken out of the glovebox. A CO gas cylinder with a pressure regulator was connected to the reactor. The reactor was pressurized with $\mathrm{CO}$ gas to 6 atm and released after $5 \mathrm{~min}$. This procedure was repeated three times, after which the reaction was stirred at 60 ${ }^{\circ} \mathrm{C}$ for $12 \mathrm{~h}$. Then, the reaction was cooled to room temperature and the $\mathrm{CO}$ gas was released. The crude reaction mixture was diluted with $\mathrm{Et}_{2} \mathrm{O}(20 \mathrm{~mL})$ and aqueous $\mathrm{NaOH}(1 \mathrm{~N}, 10 \mathrm{~mL})$. The organic phase was separated and the aqueous layer was extracted twice with $\mathrm{Et}_{2} \mathrm{O}(2 \times 10 \mathrm{~mL})$. The combined organic layers were washed with brine, dried over $\mathrm{MgSO}_{4}$, filtered and concentrated on a rotary evaporator. The residue was purified by silica gel chromatography (hexane/ethyl acetate) to afford the corresponding acylsilanes. 


$$
\psi_{6}^{\mathrm{O}} \mathrm{SiMe}_{2} \mathrm{Ph}
$$

1-(Dimethyl(phenyl)silyl)nonan-1-one (2a). The title compound was synthesized according to general procedure using 1-iodooctane $(48 \mathrm{mg}, 0.2 \mathrm{mmol})$. The crude material was purified by silica gel chromatography (hexane /ethyl acetate $=20: 1$ ) to afford the title compound as a colorless oil ( $45 \mathrm{mg}, 82 \%$ yield).

1H NMR (400 MHz, $\left.\mathrm{CDCl}_{3}\right) \delta 7.56-7.55(\mathrm{~m}, 2 \mathrm{H}), 7.44-7.35(\mathrm{~m}, 3 \mathrm{H}), 2.56(\mathrm{t}, J=7.3 \mathrm{~Hz}, 2 \mathrm{H}), 1.49$ $-1.40(\mathrm{~m}, 2 \mathrm{H}), 1.28-1.18(\mathrm{~m}, 10 \mathrm{H}), 0.87$ (t, $J=7.1 \mathrm{~Hz}, 3 \mathrm{H}), 0.49(\mathrm{~s}, 6 \mathrm{H})$.

${ }^{13} \mathrm{C}$ NMR $\left(101 \mathrm{MHz}, \mathrm{CDCl}_{3}\right) \delta$ 246.5, 134.6, 133.9, 129.8, 128.1, 48.8, 31.8, 29.3, 29.2, 29.1, 22.6, 22.2, 14.0, -4.7.

IR (neat) 2924, 2854, 1642, 1248, 1110, 834, 816, 779, 733, $698 \mathrm{~cm}^{-1}$.

HRMS (ESI) Calcd. $\mathrm{C}_{17} \mathrm{H}_{28} \mathrm{ONaSi}$ for ([M+Na]+): 299.1802; Found: 299.1804.

2.0 mmol Scale: In a glovebox, NaOPh (696 mg, 6.0 mmol, 3.0 equiv), IPrCuCl (97 mg, 0.2 mmol, $10 \mathrm{~mol} \%)$ and 1,4-dioxane $(40.0 \mathrm{~mL}$ ) were added to a $80-\mathrm{mL}$ vial with a $2.0 \mathrm{~cm}$ stir bar. The mixture was stirred at room temperature for 2 min before $\mathrm{PhMe}_{2}$ Si-Bpin (789 mg, $3.0 \mathrm{mmol}, 1.5$ equiv) and 1-iodooctane (480 mg, $2.0 \mathrm{mmol}, 1.0$ equiv) were added sequentially at room temperature. The vial was then placed in an aluminum rack within a Parr pressure reactor. Next, the reactor was closed and taken out of the glovebox. A CO gas cylinder with a pressure regulator was connected to the reactor. The reactor was pressurized with $\mathrm{CO}$ gas to $6 \mathrm{~atm}$ and released after $5 \mathrm{~min}$. This procedure was repeated three times, after which the reaction stirred at $60{ }^{\circ} \mathrm{C}$ for $12 \mathrm{~h}$. Then, the reaction was cooled to room temperature and the $\mathrm{CO}$ gas was released. The crude reaction mixture was diluted with $\mathrm{Et}_{2} \mathrm{O}(40 \mathrm{~mL})$ and aqueous $\mathrm{NaOH}(1 \mathrm{~N}, 20$ $\mathrm{mL})$. The organic phase was separated and the aqueous layer was extracted twice with $\mathrm{Et}_{2} \mathrm{O}(2 \times 20 \mathrm{~mL})$. The combined organic layers were washed with brine, dried over $\mathrm{MgSO}_{4}$, filtered and concentrated on a rotary evaporator. The crude material was purified by silica gel chromatography (hexane /ethyl acetate = $20: 1)$ to afford the title compound as a colorless oil ( $431 \mathrm{mg}, 78 \%$ yield).

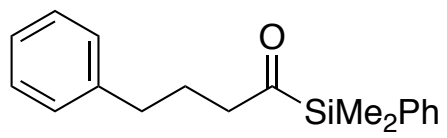

1-(Dimethyl(phenyl)silyl)-4-phenylbutan-1-one (2b). The title compound was synthesized according to general procedure using1-iodo-3-phenylpropane (49 $\mathrm{mg}, 0.2 \mathrm{mmol}$ ). The crude material was purified by silica gel chromatography (hexane /ethyl acetate $=20: 1$ ) to afford the title compound as a colorless oil (51 mg, 91\% yield).

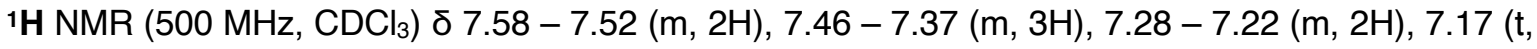
$J=7.3 \mathrm{~Hz}, 1 \mathrm{H}), 7.07(\mathrm{~d}, J=7.0 \mathrm{~Hz}, 2 \mathrm{H}), 2.60(\mathrm{t}, J=7.1 \mathrm{~Hz}, 2 \mathrm{H}), 2.53-2.48(\mathrm{~m}, 2 \mathrm{H}), 1.82-1.77(\mathrm{~m}, 2 \mathrm{H})$, $0.48(\mathrm{~d}, J=3.3 \mathrm{~Hz}, 6 \mathrm{H})$. 
${ }^{13} \mathrm{C}$ NMR $\left(126 \mathrm{MHz}, \mathrm{CDCl}_{3}\right) \delta 246.0,141.8,134.4,134.0,129.9,128.4,128.3,128.2,125.8,47.9$, 35.1, 23.7, -4.8 .

IR (neat) 3025, 2943, 1640, 1248, 1110, 834, 815, 779, 733, $697 \mathrm{~cm}^{-1}$.

HRMS (ESI) Calcd. for $\mathrm{C}_{18} \mathrm{H}_{22} \mathrm{ONaSi}\left([\mathrm{M}+\mathrm{Na}]^{+}\right)$: 305.1333; Found: 305.1338 .<smiles>CC(C)CCC(C)C(=O)Oc1ccccc1</smiles>

7-(Benzyloxy)-1-(dimethyl(phenyl)silyl)heptan-1-one (2c). The title compound was synthesized according to general procedure using (((6-iodohexyl)oxy)methyl)benzene $(64 \mathrm{mg}, 0.2 \mathrm{mmol})$. The crude material was purified by silica gel chromatography (hexane lethyl acetate $=10: 1$ ) to afford the title compound as a colorless oil (51.3 $\mathrm{mg}, 72 \%$ yield).

1H NMR (500 MHz, $\left.\mathrm{CDCl}_{3}\right) \delta 7.57-7.55(\mathrm{~m}, 2 \mathrm{H}), 7.45-7.25(\mathrm{~m}, 8 \mathrm{H}), 4.49(\mathrm{~s}, 2 \mathrm{H}), 3.43$ (t, J=6.6 $\mathrm{Hz}, 2 \mathrm{H}), 2.57(\mathrm{t}, J=7.3 \mathrm{~Hz}, 2 \mathrm{H}), 1.60-1.52(\mathrm{~m}, 2 \mathrm{H}), 1.50-1.44(\mathrm{~m}, 2 \mathrm{H}), 1.32-1.25(\mathrm{~m}, 2 \mathrm{H}), 1.22-1.17$ $(\mathrm{m}, 2 \mathrm{H}), 0.50(\mathrm{~s}, 6 \mathrm{H})$.

${ }^{13} \mathrm{C}$ NMR $\left(126 \mathrm{MHz}, \mathrm{CDCl}_{3}\right) \delta 246.4,138.6,134.5,133.9,129.8,128.3,128.1,127.6,127.4,72.8$, 70.3, 48.7, 29.5, 29.0, 25.9, 22.0, -4.8.

IR (neat) 2933, 2855, 1641, 1248, 1109, 834, 817, 780, 733, $697 \mathrm{~cm}^{-1}$.

HRMS (ESI) Calcd. for $\mathrm{C}_{22} \mathrm{H}_{31} \mathrm{O}_{2} \mathrm{Si}([\mathrm{M}+\mathrm{H}]+)$ ): 355.2088; Found: 355.2091.<smiles>CC(C)(C)CC(=O)Sc1ccccc1</smiles>

7-Chloro-1-(dimethyl(phenyl)silyl)heptan-1-one (2d). The title compound was synthesized according to general procedure using 1-chloro-6-iodohexane (49 mg, $0.2 \mathrm{mmol})$. The crude material was purified by silica gel chromatography (hexane /ethyl acetate $=20: 1$ ) to afford the title compound as a colorless oil (42 mg, 75\% yield).

${ }^{1} \mathbf{H}$ NMR $\left(500 \mathrm{MHz}, \mathrm{CDCl}_{3}\right) \delta 7.55-7.54(\mathrm{~m}, 2 \mathrm{H}), 7.45-7.36(\mathrm{~m}, 3 \mathrm{H}), 3.47(\mathrm{t}, J=6.7 \mathrm{~Hz}, 2 \mathrm{H}), 2.56$ $(\mathrm{t}, J=7.2 \mathrm{~Hz}, 2 \mathrm{H}), 1.74-1.65(\mathrm{~m}, 2 \mathrm{H}), 1.51-1.42(\mathrm{~m}, 2 \mathrm{H}), 1.38-1.29(\mathrm{~m}, 2 \mathrm{H}), 1.21-1.15(\mathrm{~m}, 2 \mathrm{H}), 0.49$ $(\mathrm{s}, 6 \mathrm{H})$.

${ }^{13} \mathrm{C} \operatorname{NMR}\left(126 \mathrm{MHz}, \mathrm{CDCl}_{3}\right) \delta 246.3,134.5,133.9,129.9,128.1,48.5,44.9,32.3,28.3,26.6,21.9,-$ 4.8 .

IR (neat) 2935, 2858, 1641, 1248, 1110, 835, 816, 780, 734, 699, $648 \mathrm{~cm}^{-1}$.

HRMS (ESI) Calcd. for $\mathrm{C}_{15} \mathrm{H}_{23} \mathrm{ONaSiCl}\left([\mathrm{M}+\mathrm{Na}]^{+}\right)$: 305.1099; Found: 305.1096.<smiles>CC(C)OCc1ccco1</smiles>

1-(Dimethyl(phenyl)silyl)-7-(furan-2-ylmethoxy)heptan-1-one (2e). The title compound was synthesized according to general procedure using 2-(((6-iodohexyl)oxy)methyl)furan (62 mg, $0.2 \mathrm{mmol})$. 
The crude material was purified by silica gel chromatography (hexane /ethyl acetate $=15: 1$ ) to afford the title compound as a colorless oil ( $48 \mathrm{mg}, 70 \%$ yield).

1H NMR (400 MHz, $\left.\mathrm{CDCl}_{3}\right) \delta 7.59-7.52(\mathrm{~m}, 2 \mathrm{H}), 7.45-7.35(\mathrm{~m}, 5 \mathrm{H}), 6.40(\mathrm{~d}, J=1.2 \mathrm{~Hz}, 1 \mathrm{H}), 4.34$ (s, 2H), $3.38(\mathrm{t}, J=6.6 \mathrm{~Hz}, 2 \mathrm{H}), 2.55(\mathrm{t}, J=7.2 \mathrm{~Hz}, 2 \mathrm{H}), 1.54-1.48(\mathrm{~m}, 2 \mathrm{H}), 1.47-1.41(\mathrm{~m}, 2 \mathrm{H}), 1.28-$ $1.23(\mathrm{~m}, 2 \mathrm{H}), 1.22-1.13(\mathrm{~m}, 2 \mathrm{H}), 0.48(\mathrm{~s}, 6 \mathrm{H})$.

${ }^{13} \mathrm{C}$ NMR $\left(101 \mathrm{MHz}, \mathrm{CDCl}_{3}\right) \delta 246.4,143.2,140.5,134.6,133.9,129.8,128.1,127.6,110.3,70.1$, 64.0, 48.7, 29.5, 29.0, 26.0, 22.1, -4.8.

IR (neat) 2933, 2857, 1640, 1249, 1109, 780, 733, $699 \mathrm{~cm}^{-1}$.

HRMS (ESI) Calcd. for $\mathrm{C}_{20} \mathrm{H}_{29} \mathrm{O}_{3} \mathrm{Si}([\mathrm{M}+\mathrm{H}]+)$ : 345.1881; Found: 345.1881 .<smiles>CC(CC(C)(C)OCCc1cccs1)SC(=O)c1ccccc1</smiles>

1-(Dimethyl(phenyl)silyl)-7-(2-(thiophen-2-yl)ethoxy)heptan-1-one (2f). The title compound was synthesized according to general procedure using 2-(2-((6-iodohexyl)oxy)ethyl)thiophene (67 mg, 0.2 $\mathrm{mmol}$ ). The crude material was purified by silica gel chromatography (hexane /ethyl acetate $=15: 1$ ) to afford the title compound as a colorless oil ( $58 \mathrm{mg}, 77 \%$ yield).

1H NMR (500 MHz, $\left.\mathrm{CDCl}_{3}\right) \delta 7.56-7.55(\mathrm{~m}, 2 \mathrm{H}), 7.44-7.36(\mathrm{~m}, 3 \mathrm{H}), 7.12(\mathrm{~d}, J=5.1 \mathrm{~Hz}, 1 \mathrm{H}), 6.95$ $-6.90(\mathrm{~m}, 1 \mathrm{H}), 6.84(\mathrm{~d}, J=2.5 \mathrm{~Hz}, 1 \mathrm{H}), 3.63(\mathrm{t}, J=6.9 \mathrm{~Hz}, 2 \mathrm{H}), 3.40(\mathrm{t}, J=6.6 \mathrm{~Hz}, 2 \mathrm{H}), 3.08(\mathrm{t}, J=6.8 \mathrm{~Hz}$, $2 \mathrm{H}), 2.56(\mathrm{t}, J=7.3 \mathrm{~Hz}, 2 \mathrm{H}), 1.53-1.49(\mathrm{~m}, 2 \mathrm{H}), 1.47-1.43(\mathrm{~m}, 2 \mathrm{H}), 1.28-1.24(\mathrm{~m}, 2 \mathrm{H}), 1.21-1.15(\mathrm{~m}$, $2 \mathrm{H}), 0.49(\mathrm{~s}, 6 \mathrm{H})$.

${ }^{13} \mathrm{C} \mathrm{NMR}\left(126 \mathrm{MHz}, \mathrm{CDCl}_{3}\right) \delta 246.5,141.4,134.6,133.9,129.8,128.1,126.6,125.0,123.5,71.3$, $70.9,48.7,30.5,29.4,29.0,25.9,22.1,-4.8$.

IR (neat) 2933, 2856, 1641, 1109, 817, 780, 734, $696 \mathrm{~cm}^{-1}$.

HRMS (ESI) Calcd. for $\mathrm{C}_{21} \mathrm{H}_{31} \mathrm{O}_{2} \mathrm{SiS}\left([\mathrm{M}+\mathrm{H}]^{+}\right)$: 375.1809; Found: 375.1811.<smiles>CC(C)CC(C)C(=O)Sc1ccccc1</smiles>

1-(Dimethyl(phenyl)silyl)-7-(1H-indol-1-yl)heptan-1-one (2g). The title compound was synthesized according to general procedure using 1-(6-iodohexyl)-1H-indole (65 mg, $0.2 \mathrm{mmol})$. This reaction was performed at room temperature. The crude material was purified by silica gel chromatography (hexane /ethyl acetate $=10: 1$ ) to afford the title compound as a colorless oil ( $36 \mathrm{mg}, 50 \%$ yield).

1H NMR (500 MHz, $\left.\mathrm{CDCl}_{3}\right) \delta 7.64(\mathrm{~d}, J=7.9 \mathrm{~Hz}, 1 \mathrm{H}), 7.56-7.54(\mathrm{~m}, 2 \mathrm{H}), 7.44-7.36(\mathrm{~m}, 3 \mathrm{H}), 7.33$ $(\mathrm{d}, J=8.2 \mathrm{~Hz}, 1 \mathrm{H}), 7.21(\mathrm{t}, J=7.6 \mathrm{~Hz}, 1 \mathrm{H}), 7.11(\mathrm{t}, J=7.2 \mathrm{~Hz}, 1 \mathrm{H}), 7.07(\mathrm{~d}, J=3.1 \mathrm{~Hz}, 1 \mathrm{H}), 6.49(\mathrm{~d}, J=$ $2.9 \mathrm{~Hz}, 1 \mathrm{H}), 4.07(\mathrm{t}, J=7.1 \mathrm{~Hz}, 2 \mathrm{H}), 2.54(\mathrm{t}, J=7.2 \mathrm{~Hz}, 2 \mathrm{H}), 1.80-1.73(\mathrm{~m}, 2 \mathrm{H}), 1.49-1.41(\mathrm{~m}, 2 \mathrm{H}), 1.25$ $-1.17(\mathrm{~m}, 4 \mathrm{H}), 0.49(\mathrm{~s}, 6 \mathrm{H})$.

${ }^{13} \mathrm{C}$ NMR $\left(126 \mathrm{MHz}, \mathrm{CDCl}_{3}\right) \delta 246.3,135.9,134.9,133.9,129.9,128.5,128.1,127.7,121.2,120.9$, $119.1,109.3,100.8,48.5,46.2,29.9,28.7,26.7,21.9,-4.8$. 
IR (neat) 2931, 2856, 1640, 1248, 1110, 834, 816, 737, $700 \mathrm{~cm}^{-1}$.

HRMS (ESI) Calcd. for $\mathrm{C}_{23} \mathrm{H}_{30} \mathrm{NOSi}([\mathrm{M}+\mathrm{H}]+)$ : 364.2092; Found: 364.2097.<smiles>CC(C)CC(=O)Sc1ccccc1</smiles>

1-(Dimethyl(phenyl)silyl)-7-(4-(trifluoromethyl)phenoxy)heptan-1-one (2h). The title compound was synthesized according to general procedure using 1-((6-iodohexyl)oxy)-4-(trifluoromethyl)benzene (74 $\mathrm{mg}, 0.2 \mathrm{mmol}$ ). The crude material was purified by silica gel chromatography (hexane /ethyl acetate $=20: 1$ ) to afford the title compound as a colorless oil (59 $\mathrm{mg}, 73 \%$ yield).

${ }^{1} \mathrm{H}$ NMR $\left(500 \mathrm{MHz}, \mathrm{CDCl}_{3}\right) \delta 7.59-7.50(\mathrm{~m}, 4 \mathrm{H}), 7.45-7.36(\mathrm{~m}, 3 \mathrm{H}), 6.92(\mathrm{~d}, J=8.6 \mathrm{~Hz}, 2 \mathrm{H}), 3.93$ (t, J=6.5 Hz, 2H), $2.59(\mathrm{t}, J=7.2 \mathrm{~Hz}, 2 \mathrm{H}), 1.75-1.70(\mathrm{~m}, 2 \mathrm{H}), 1.52-1.46(\mathrm{~m}, 2 \mathrm{H}), 1.40-1.35(\mathrm{~m}, 2 \mathrm{H})$, $1.27-1.20(\mathrm{~m}, 2 \mathrm{H}), 0.49(\mathrm{~s}, 6 \mathrm{H})$.

${ }^{13} \mathrm{C}$ NMR $\left(126 \mathrm{MHz}, \mathrm{CDCl}_{3}\right) \delta 246.4,161.5,134.5,134.0,129.9,128.2,126.8$ (q, Jc-F $\left.=3.8 \mathrm{~Hz}\right)$, $124.5\left(q, J_{C-F}=270.9 \mathrm{~Hz}\right), 122.6\left(q, J_{C-F}=32.8 \mathrm{~Hz}\right), 114.4,68.0,48.6,28.8,25.8,22.0,-4.8$.

$19 \mathrm{~F}$ NMR $\left(377 \mathrm{MHz}, \mathrm{CDCl}_{3}\right) \delta-61.87$.

IR (neat) 2938, 1641, 1615, 1325, 1255, 1108, 1067, 834, 734, $699 \mathrm{~cm}^{-1}$.

HRMS (ESI) Calcd. for $\mathrm{C}_{22} \mathrm{H}_{27} \mathrm{~F}_{3} \mathrm{O}_{2} \mathrm{NaSi}\left([\mathrm{M}+\mathrm{Na}]^{+}\right)$: 431.1625; Found: 431.1621.<smiles>COC(=O)c1ccc(OCC(C)CC(=O)Sc2ccccc2)cc1</smiles>

Methyl 4-((7-(dimethyl(phenyl)silyl)-7-oxoheptyl)oxy)benzoate (2i). The title compound was synthesized according to general procedure using methyl 4-((6-iodohexyl)oxy)benzoate (72 mg, $0.2 \mathrm{mmol})$. The crude material was purified by silica gel chromatography (hexane /ethyl acetate $=10: 1$ ) to afford the title compound as a white solid (60 $\mathrm{mg}, 75 \%$ yield).

1H NMR (500 MHz, $\left.\mathrm{CDCl}_{3}\right) \delta 7.97(\mathrm{~d}, J=8.8 \mathrm{~Hz}, 2 \mathrm{H}), 7.62-7.51(\mathrm{~m}, 2 \mathrm{H}), 7.46-7.34(\mathrm{~m}, 3 \mathrm{H}), 6.87$ (dd, $J=7.0,1.9 \mathrm{~Hz}, 2 \mathrm{H}$ ), 3.94 (t, $J=6.5 \mathrm{~Hz}, 2 \mathrm{H}$ ), 3.88 (s, 3H), 2.58 (t, $J=7.2 \mathrm{~Hz}, 2 \mathrm{H}), 1.75-1.69(\mathrm{~m}, 2 \mathrm{H})$, $1.54-1.44(\mathrm{~m}, 2 \mathrm{H}), 1.40-1.33(\mathrm{~m}, 2 \mathrm{H}), 1.26-1.21(\mathrm{~m}, 2 \mathrm{H}), 0.49(\mathrm{~s}, 6 \mathrm{H})$.

${ }^{13} \mathrm{C} \mathrm{NMR}\left(126 \mathrm{MHz}, \mathrm{CDCl}_{3}\right) \delta 246.3,166.9,162.8,134.5,133.9,131.5,129.8,128.1,122.3,114.00$, $67.9,51.8,48.5,28.8,25.7,21.9,-4.8$.

IR (neat) 2940, 2863, 1711, 1641, 1605, 1256, 1166, 1106, $697 \mathrm{~cm}^{-1}$.

HRMS (ESI) Calcd. for $\mathrm{C}_{23} \mathrm{H}_{30} \mathrm{O}_{4} \mathrm{NaSi}([\mathrm{M}+\mathrm{Na}]+)$ ): 421.1806 ; Found: 421.1803.<smiles>CC(C)CC(C)C(=O)Oc1ccc(C#N)cc1</smiles> 
4-((7-(Dimethyl(phenyl)silyl)-7-oxoheptyl)oxy)benzonitrile (2j). The title compound was synthesized according to general procedure using 4-((6-lodohexyl)oxy)benzonitrile (66 mg, $0.2 \mathrm{mmol})$. The crude material was purified by silica gel chromatography (hexane /ethyl acetate $=10: 1$ ) to afford the title compound as a colorless oil ( $61 \mathrm{mg}, 83 \%$ yield).

1H NMR (500 MHz, $\left.\mathrm{CDCl}_{3}\right) \delta 7.57-7.53(\mathrm{~m}, 4 \mathrm{H}), 7.44-7.35(\mathrm{~m}, 3 \mathrm{H}), 6.94-6.88(\mathrm{~m}, 2 \mathrm{H}), 3.93(\mathrm{t}$, $J=6.5 \mathrm{~Hz}, 2 \mathrm{H}), 2.57(\mathrm{t}, J=7.2 \mathrm{~Hz}, 2 \mathrm{H}), 1.75-1.68(\mathrm{~m}, 2 \mathrm{H}), 1.52-1.45(\mathrm{~m}, 2 \mathrm{H}), 1.40-1.33(\mathrm{~m}, 2 \mathrm{H})$, $1.27-1.19(\mathrm{~m}, 2 \mathrm{H}), 0.48(\mathrm{~s}, 6 \mathrm{H})$.

${ }^{13} \mathrm{C} \mathrm{NMR}\left(126 \mathrm{MHz}, \mathrm{CDCl}_{3}\right) \delta 246.3,162.4,134.5,133.9,129.8,128.1,119.3,115.1,103.6,68.2$, 48.5, 28.8, 28.7, 25.7, 21.9, -4.8.

IR (neat) 2936, 2859, 2223, 1640, 1605, 1507, 1256, 1170, 833, 780, 735, $700 \mathrm{~cm}^{-1}$.

HRMS (ESI) Calcd. for $\mathrm{C}_{22} \mathrm{H}_{27} \mathrm{NO}_{2} \mathrm{NaSi}\left([\mathrm{M}+\mathrm{Na}]^{+}\right)$: 388.1704; Found: 388.1713.

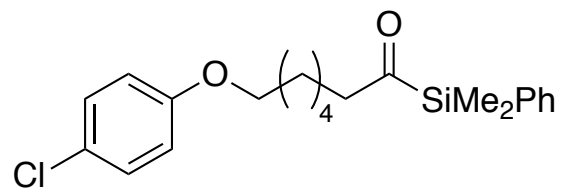

7-(4-Chlorophenoxy)-1-(dimethyl(phenyl)silyl)heptan-1-one (2k). The title compound was synthesized according to general procedure using 1-chloro-4-((6-iodohexyl)oxy)benzene (68 mg, 0.2 $\mathrm{mmol}$ ). The crude material was purified by silica gel chromatography (hexane /ethyl acetate $=20: 1$ ) to afford the title compound as a colorless oil ( $31 \mathrm{mg}, 83 \%$ yield).

1H NMR (500 MHz, $\left.\mathrm{CDCl}_{3}\right) \delta 7.56-7.54(\mathrm{~m}, 2 \mathrm{H}), 7.45-7.35(\mathrm{~m}, 3 \mathrm{H}), 7.24-7.19(\mathrm{~m}, 2 \mathrm{H}), 6.79(\mathrm{~d}$, $J=8.9 \mathrm{~Hz}, 2 \mathrm{H}), 3.86(\mathrm{t}, J=6.5 \mathrm{~Hz}, 2 \mathrm{H}), 2.57(\mathrm{t}, J=7.2 \mathrm{~Hz}, 2 \mathrm{H}), 1.72-1.66(\mathrm{~m}, 2 \mathrm{H}), 1.52-1.45(\mathrm{~m}, 2 \mathrm{H})$, $1.40-1.33(\mathrm{~m}, 2 \mathrm{H}), 1.25-1.20(\mathrm{~m}, 2 \mathrm{H}), 0.49(\mathrm{~s}, 6 \mathrm{H})$.

${ }^{13} \mathrm{C} \mathrm{NMR}\left(126 \mathrm{MHz}, \mathrm{CDCl}_{3}\right) \delta 246.4,157.7,134.5,134.0,129.9,129.2,128.2,125.3,115.7,68.1$, $48.6,28.9,28.9,25.8,22.0,-4.8$.

IR (neat) 2937, 2859, 1641, 1491, 1243, 822, 780, 734, $699 \mathrm{~cm}^{-1}$.

HRMS (EI) Calcd. for $\mathrm{C}_{21} \mathrm{H}_{27} \mathrm{ClO}_{2} \mathrm{Si}([\mathrm{M}]+)$ : 374.1464 ; Found: 374.1460 .<smiles>CCCOc1ccc(Br)cc1</smiles>

7-(4-Bromophenoxy)-1-(dimethyl(phenyl)silyl)heptan-1-one (2l). The title compound was synthesized according to general procedure using 1-bromo-4-((6-iodohexyl)oxy)benzene (77 mg, 0.2 $\mathrm{mmol}$ ). The crude material was purified by silica gel chromatography (hexane /ethyl acetate $=20: 1$ ) to afford the title compound as a colorless oil ( $30 \mathrm{mg}, 72 \%$ yield).

${ }^{1} \mathrm{H}$ NMR $\left(500 \mathrm{MHz}, \mathrm{CDCl}_{3}\right) \delta 7.57-7.53(\mathrm{~m}, 2 \mathrm{H}), 7.44-7.32(\mathrm{~m}, 5 \mathrm{H}), 6.75(\mathrm{~d}, J=8.9 \mathrm{~Hz}, 2 \mathrm{H}), 3.85$ (t, $J=6.5 \mathrm{~Hz}, 2 \mathrm{H}), 2.57(\mathrm{t}, J=7.2 \mathrm{~Hz}, 2 \mathrm{H}), 1.72-1.66(\mathrm{~m}, 2 \mathrm{H}), 1.51-1.44(\mathrm{~m}, 2 \mathrm{H}), 1.38-1.32(\mathrm{~m}, 2 \mathrm{H})$, $1.25-1.20(\mathrm{~m}, 2 \mathrm{H}), 0.49(\mathrm{~s}, 6 \mathrm{H})$. 
${ }^{13} \mathrm{C} \mathrm{NMR}\left(126 \mathrm{MHz}, \mathrm{CDCl}_{3}\right) \delta 246.4,158.2,134.5,134.0,132.2,129.9,128.2,116.3,112.6,68.0$, 48.6, 28.9, 28.9, 25.8, 22.0, -4.8.

IR (neat) 2936, 2858, 1640, 1488, 1242, 1110, 819, 780, 734, $690 \mathrm{~cm}^{-1}$.

HRMS (ESI) Calcd. for $\mathrm{C}_{21} \mathrm{H}_{27} \mathrm{O}_{2} \mathrm{NaSiBr}\left([\mathrm{M}+\mathrm{Na}]^{+}\right)$: 441.0856; Found: 441.0850.<smiles>CCCCC(=O)CCCCOc1ccc(I)cc1</smiles>

1-(Dimethyl(phenyl)silyl)-7-(4-iodophenoxy)heptan-1-one $(\mathbf{2 m})$. The title compound was synthesized according to general procedure using 1-iodo-4-((6-iodohexyl)oxy)benzene (86 mg, $0.2 \mathrm{mmol})$. The crude material was purified by silica gel chromatography (hexane /ethyl acetate $=20: 1$ ) to afford a mixture of the title compound and the deiodonized acylsilane as a colorless oil (34 $\mathrm{mg}$ ). The ratio of the title compound and the deiodonized acylsilane was determined to be 2.4:1 by ${ }^{1} \mathrm{H}$ NMR analysis of the isolated compound.

${ }^{1} \mathrm{H}$ NMR $\left(500 \mathrm{MHz}, \mathrm{CDCl}_{3}\right) \delta$ 7.56-7.52 (m, 4H), $7.41-7.37(\mathrm{~m}, 3 \mathrm{H}), 6.65(\mathrm{~d}, J=8.7 \mathrm{~Hz}, 2 \mathrm{H}), 3.85$ $(\mathrm{t}, J=6.5 \mathrm{~Hz}, 2 \mathrm{H}), 2.57(\mathrm{t}, J=7.2 \mathrm{~Hz}, 2 \mathrm{H}), 1.74-1.65(\mathrm{~m}, 2 \mathrm{H}), 1.52-1.44(\mathrm{~m}, 2 \mathrm{H}), 1.40-1.32(\mathrm{~m}, 2 \mathrm{H})$, $1.26-1.19(\mathrm{~m}, 2 \mathrm{H}), 0.49(\mathrm{~s}, 6 \mathrm{H})$.

${ }_{13} \mathrm{C} \mathrm{NMR}\left(126 \mathrm{MHz}, \mathrm{CDCl}_{3}\right) \delta 246.4,158.9,138.1,134.5,133.9,129.8,129.4,128.1,120.4,116.9$, 114.4, 82.4, 67.9, 48.6, 28.8, 25.8, 22.0, -4.8.

IR (neat) 2936, 2858, 1641, 1486, 1244, 819, $700 \mathrm{~cm}^{-1}$.

HRMS (ESI) Calcd. for $\mathrm{C}_{21} \mathrm{H}_{27} \mathrm{O}_{2} \mathrm{NaSil}\left([\mathrm{M}+\mathrm{Na}]^{+}\right)$: 489.0718; Found: 489.0720 .<smiles>C=CCCC(C)C(=O)Oc1ccccc1</smiles>

1-(Dimethyl(phenyl)silyl)dec-9-en-1-one (2n). The title compound was synthesized according to general procedure using 10-iodo-1-decene (53 mg, $0.2 \mathrm{mmol})$. The crude material was purified by silica gel chromatography (hexane /ethyl acetate $=20: 1$ ) to afford the title compound as a colorless oil (47 $\mathrm{mg}, 81 \%$ yield).

${ }^{1} \mathbf{H}$ NMR $\left(500 \mathrm{MHz}, \mathrm{CDCl}_{3}\right) \delta 7.58-7.53(\mathrm{~m}, 2 \mathrm{H}), 7.44-7.36(\mathrm{~m}, 3 \mathrm{H}), 5.80$ (ddt, $J=13.0,9.7,6.6$ $\mathrm{Hz}, 1 \mathrm{H}), 4.99(\mathrm{~d}, J=17.1 \mathrm{~Hz}, 1 \mathrm{H}), 4.93(\mathrm{~d}, J=10.1 \mathrm{~Hz}, 1 \mathrm{H}), 2.55(\mathrm{t}, J=7.2 \mathrm{~Hz}, 2 \mathrm{H}), 2.04-2.00(\mathrm{~m}, 2 \mathrm{H})$, $1.47-1.41(\mathrm{~m}, 2 \mathrm{H}), 1.38-1.32(\mathrm{~m}, 2 \mathrm{H}), 1.26-1.15(\mathrm{~m}, 8 \mathrm{H}), 0.49(\mathrm{~s}, 6 \mathrm{H})$.

${ }_{13} \mathrm{C} \mathrm{NMR}\left(126 \mathrm{MHz}, \mathrm{CDCl}_{3}\right) \delta 246.6,139.2,134.6,133.9,129.8,128.1,114.1,48.8,33.7,29.3,29.2$, 29.2, 29.0, 28.8, 22.1, -4.8.

IR (neat) 2925, 2853, 1641, 1248, 1110, 834, 816, 779, 733, $698 \mathrm{~cm}^{-1}$.

HRMS (ESI) Calcd. for $\mathrm{C}_{20} \mathrm{H}_{30} \mathrm{ONaSi}\left([\mathrm{M}+\mathrm{Na}]^{+}\right)$: 325.1959; Found: 325.1967. 
<smiles>CC(C)C(=O)Oc1ccccc1</smiles>

1-(Dimethyl(phenyl)silyl)-2-methylpropan-1-one (20). The title compound was synthesized according to general procedure using 2-iodopropane (34 $\mathrm{mg}, 0.2 \mathrm{mmol})$. The crude material was purified by silica gel chromatography (hexane /ethyl acetate $=20: 1$ ) to afford the title compound as a colorless oil (39 mg, 98\% yield).

1H NMR (500 MHz, $\left.\mathrm{CDCl}_{3}\right) \delta 7.59-7.54(\mathrm{~m}, 2 \mathrm{H}), 7.43-7.35(\mathrm{~m}, 3 \mathrm{H}), 2.90$ (hept, $\left.J=6.9 \mathrm{~Hz}, 1 \mathrm{H}\right)$, $0.92(\mathrm{~d}, J=6.9 \mathrm{~Hz}, 6 \mathrm{H}), 0.51(\mathrm{~s}, 6 \mathrm{H})$.

${ }^{13} \mathrm{C}$ NMR $\left(126 \mathrm{MHz}, \mathrm{CDCl}_{3}\right) \delta 248.6,135.0,133.9,129.7,128.1,45.3,16.4,-4.1$.

IR (neat) 2965, 1637, 1248, 1111, 833, 810, 779, 734, $699 \mathrm{~cm}^{-1}$.

HRMS (ESI) Calcd. for $\mathrm{C}_{12} \mathrm{H}_{18} \mathrm{ONaSi}\left([\mathrm{M}+\mathrm{Na}]^{+}\right)$: 229.1020; Found: 229.1024.

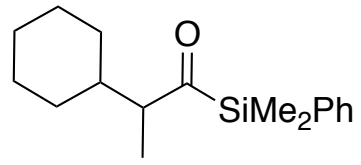

2-Cyclohexyl-1-(dimethyl(phenyl)silyl)propan-1-one (2p). The title compound was synthesized according to general procedure using 1-cyclohexyliodoethane (48 $\mathrm{mg}, 0.2 \mathrm{mmol}$ ). The crude material was purified by silica gel chromatography (hexane /ethyl acetate $=20: 1$ ) to afford the title compound as a colorless oil (49 $\mathrm{mg}, 90 \%$ yield).

${ }_{1}^{1} \mathrm{H}$ NMR $\left(400 \mathrm{MHz}, \mathrm{CDCl}_{3}\right) \delta 7.60-7.52(\mathrm{~m}, 2 \mathrm{H}), 7.42-7.34(\mathrm{~m}, 3 \mathrm{H}), 2.69(\mathrm{p}, J=6.8 \mathrm{~Hz}, 1 \mathrm{H}), 1.66$ $-1.57(\mathrm{~m}, 4 \mathrm{H}), 1.38-1.36(\mathrm{~m}, 2 \mathrm{H}), 1.16-1.00(\mathrm{~m}, 3 \mathrm{H}), 0.95-0.82(\mathrm{~m}, 2 \mathrm{H}), 0.79$ (d, J = $7.0 \mathrm{~Hz}, 3 \mathrm{H})$, $0.50(\mathrm{~s}, 3 \mathrm{H}), 0.49(\mathrm{~s}, 3 \mathrm{H})$.

${ }^{13} \mathrm{C}$ NMR $\left(101 \mathrm{MHz}, \mathrm{CDCl}_{3}\right) \delta 249.6,135.0,134.0,129.7,128.0,56.4,37.8,32.2,28.6,26.5,26.3$, $26.2,10.6,-4.3,-4.4$.

IR (neat) 2923, 2851, 1637, 1448, 1247, 1109, 833, 812, 778, 735, $699 \mathrm{~cm}^{-1}$.

HRMS (ESI) Calcd. for $\mathrm{C}_{17} \mathrm{H}_{26} \mathrm{ONaSi}\left([\mathrm{M}+\mathrm{Na}]^{+}\right)$: 297.1646; Found: 297.1646.<smiles>CC(=O)C1CCCC1</smiles>

Cyclopentyl(dimethyl(phenyl)silyl)methanone (2q). The title compound was synthesized according to general procedure using iodocyclopentane (39 $\mathrm{mg}, 0.2 \mathrm{mmol}$ ). The crude material was purified by silica gel chromatography (hexane /ethyl acetate $=20: 1$ ) to afford the title compound as a colorless oil (45 mg, 97\% yield).

${ }^{1} \mathrm{H}$ NMR $\left(500 \mathrm{MHz}, \mathrm{CDCl}_{3}\right) \delta 7.57-7.55(\mathrm{~m}, 2 \mathrm{H}), 7.49-7.35(\mathrm{~m}, 3 \mathrm{H}), 3.23-3.17(\mathrm{~m}, 1 \mathrm{H}), 1.73-$ $1.63(\mathrm{~m}, 2 \mathrm{H}), 1.61-1.44(\mathrm{~m}, 6 \mathrm{H}), 0.50(\mathrm{~s}, 6 \mathrm{H})$.

${ }^{13} \mathrm{C} \mathrm{NMR}\left(126 \mathrm{MHz}, \mathrm{CDCl}_{3}\right) \delta 246.6,135.0,134.0,129.7,128.0,56.3,26.7,25.9,-4.3$. 
IR (neat) 2954, 2866, 1635, 1248, 1109, 832, 808, 779, 734, $699 \mathrm{~cm}^{-1}$.

HRMS (El) Calcd. for $\mathrm{C}_{14} \mathrm{H}_{20} \mathrm{OSi}\left([\mathrm{M}]^{+}\right)$: 232.1278; Found: 232.1291.<smiles>CC(=O)C1CCCCC1</smiles>

Cyclohexyl(dimethyl(phenyl)silyl)methanone (2r). The title compound was synthesized according to general procedure using iodocyclohexane $(42 \mathrm{mg}, 0.2 \mathrm{mmol})$. The crude material was purified by silica gel chromatography (hexane /ethyl acetate $=20: 1$ ) to afford the title compound as a colorless oil (43 mg, $87 \%$ yield).

1H NMR (500 MHz, $\left.\mathrm{CDCl}_{3}\right) \delta 7.58-7.52(\mathrm{~m}, 2 \mathrm{H}), 7.42-7.35(\mathrm{~m}, 3 \mathrm{H}), 2.73-2.63(\mathrm{~m}, 1 \mathrm{H}), 1.70-$ $1.58(\mathrm{~m}, 5 \mathrm{H}), 1.20-1.10(\mathrm{~m}, 5 \mathrm{H}), 0.49(\mathrm{~s}, 6 \mathrm{H})$.

${ }^{13} \mathrm{C}$ NMR $\left(126 \mathrm{MHz}, \mathrm{CDCl}_{3}\right) \delta 248.5,135.0,133.9,129.7,128.0,55.6,26.7,25.9,25.6,-4.1$.

IR (neat) 2927, 2852, 1635, 1247, 1109, 834, 820, 778, 733, $699 \mathrm{~cm}^{-1}$.

HRMS (El) Calcd. for $\mathrm{C}_{15} \mathrm{H}_{22} \mathrm{OSi}([\mathrm{M}]+)$ : 246.1440; Found: 246.1451.<smiles>CC(C)SC(=O)C1CCOCC1</smiles>

(Dimethyl(phenyl)silyl)(tetrahydro-2H-pyran-4-yl)methanone (2s). The title compound was synthesized according to general procedure using 4-iodo-tetrahydropyran ( $42 \mathrm{mg}, 0.2 \mathrm{mmol}$ ). The crude material was purified by silica gel chromatography (hexane /ethyl acetate $=8: 1$ ) to afford the title compound as a colorless oil ( $37 \mathrm{mg}, 84 \%$ yield).

1H NMR (500 MHz, $\left.\mathrm{CDCl}_{3}\right) \delta 7.54-7.53(\mathrm{~m}, 2 \mathrm{H}), 7.41-7.37(\mathrm{~m}, 3 \mathrm{H}), 3.95-3.82(\mathrm{~m}, 2 \mathrm{H}), 3.40-$ $3.27(\mathrm{~m}, 2 \mathrm{H}), 2.93-2.80(\mathrm{~m}, 1 \mathrm{H}), 1.55-1.50(\mathrm{~m}, 4 \mathrm{H}), 0.50(\mathrm{~s}, 6 \mathrm{H})$.

${ }^{13} \mathrm{C} \mathrm{NMR}\left(126 \mathrm{MHz}, \mathrm{CDCl}_{3}\right) \delta 246.3,134.5,133.9,129.9,128.2,67.1,52.2,26.6,-4.3$.

IR (neat) 2953, 2843, 1634, 1248, 1106, 826, 779, 735, $700 \mathrm{~cm}^{-1}$.

HRMS (El) Calcd. for $\mathrm{C}_{14} \mathrm{H}_{20} \mathrm{O}_{2} \mathrm{Si}([\mathrm{M}]+)$ : 248.1228; Found: 248.1225.<smiles>CC(C)SC(=O)C1CCN(C(C)(C)C)CC1</smiles>

tert-butyl 4-((dimethyl(phenyl)silyl)carbonyl)piperidine-1-carboxylate (2t). The title compound was synthesized according to general procedure using N-Boc-4-iodopiperidine (62 mg, $0.2 \mathrm{mmol}$ ). The crude material was purified by silica gel chromatography (hexane /ethyl acetate $=8: 1$ ) to afford the title compound as a colorless oil ( $49 \mathrm{mg}, 70 \%$ yield). 
1H NMR (500 MHz, $\left.\mathrm{CDCl}_{3}\right) \delta 7.54-7.52(\mathrm{~m}, 2 \mathrm{H}), 7.44-7.35(\mathrm{~m}, 3 \mathrm{H}), 3.96$ (brs, 2H), 2.77 (tt, $J=$ 11.1, 3.7 Hz, 1H), $2.67(\mathrm{t}, J=11.5 \mathrm{~Hz}, 2 \mathrm{H}), 1.59-1.57(\mathrm{~m}, 2 \mathrm{H}), 1.41(\mathrm{~s}, 9 \mathrm{H}), 1.39-1.30(\mathrm{~m}, 2 \mathrm{H}), 0.49$ (s, $6 \mathrm{H})$.

${ }^{13} \mathrm{C} \mathrm{NMR}\left(126 \mathrm{MHz}, \mathrm{CDCl}_{3}\right) \delta 246.7,154.6,134.4,133.9,129.9,128.2,79.4,53.1,43.1,28.4,25.9$, $-4.3$.

IR (neat) 2971, 2854, 1689, 1635, 1419, 1166, 827, 779, 735, $701 \mathrm{~cm}^{-1}$.

HRMS (ESI) Calcd. for $\mathrm{C}_{19} \mathrm{H}_{29} \mathrm{NO}_{3} \mathrm{NaSi}\left([\mathrm{M}+\mathrm{Na}]^{+}\right)$: 370.1809; Found: 370.1810.<smiles>CC(C)(C)C(=O)Oc1ccccc1</smiles>

1-(Dimethyl(phenyl)silyl)-2,2-dimethylpropan-1-one (2u). The title compound was synthesized according to general procedure using $t$-butyl bromide $(27 \mathrm{mg}, 0.2 \mathrm{mmol})$. The crude material was purified by silica gel chromatography (hexane /ethyl acetate $=20: 1$ ) to afford the title compound as a colorless oil (34 mg, 77\% yield).

1H NMR (500 MHz, CDCl $)$ ס $7.57-7.55(\mathrm{~m}, 2 \mathrm{H}), 7.42-7.34(\mathrm{~m}, 3 \mathrm{H}), 0.99(\mathrm{~s}, 9 \mathrm{H}), 0.54(\mathrm{~s}, 6 \mathrm{H})$.

${ }^{13} \mathrm{C}$ NMR $\left(126 \mathrm{MHz}, \mathrm{CDCl}_{3}\right) \delta 247.9,136.0,134.1,129.6,128.0,49.4,24.8,-2.4$.

IR (neat) 2963, 2868, 1630, 1248, 1108, 816, 778, 735, $701 \mathrm{~cm}^{-1}$.

HRMS (ESI) Calcd. for $\mathrm{C}_{13} \mathrm{H}_{20} \mathrm{ONaSi}\left([\mathrm{M}+\mathrm{Na}]^{+}\right)$: 243.1176; Found: 243.1183.<smiles>CC(C)(CCc1ccccc1)C(=O)Sc1ccccc1</smiles>

1-(Dimethyl(phenyl)silyl)-2,2-dimethyl-4-phenylbutan-1-one (2v). The title compound was synthesized according to general procedure using (3-bromo-3-methylbutyl)benzene (45 mg, $0.2 \mathrm{mmol}$ ). The crude material was purified by silica gel chromatography (hexane /ethyl acetate $=20: 1$ ) to afford the title compound as a colorless oil (57 $\mathrm{mg}, 92 \%$ yield).

${ }^{1} \mathrm{H}$ NMR $\left(500 \mathrm{MHz}, \mathrm{CDCl}_{3}\right) \delta 7.60(\mathrm{~d}, J=7.5 \mathrm{~Hz}, 2 \mathrm{H}), 7.43-7.37(\mathrm{~m}, 3 \mathrm{H}), 7.26(\mathrm{t}, J=7.4 \mathrm{~Hz}, 2 \mathrm{H})$, $7.18(\mathrm{t}, J=7.3 \mathrm{~Hz}, 1 \mathrm{H}), 7.03(\mathrm{~d}, J=7.5 \mathrm{~Hz}, 2 \mathrm{H}), 2.37-2.29(\mathrm{~m}, 2 \mathrm{H}), 1.79-1.70(\mathrm{~m}, 2 \mathrm{H}), 1.08(\mathrm{~s}, 6 \mathrm{H})$, $0.58(\mathrm{~s}, 6 \mathrm{H})$.

${ }^{13} \mathrm{C}$ NMR $\left(126 \mathrm{MHz}, \mathrm{CDCl}_{3}\right) \delta 248.0,142.3,135.8,134.1,129.7,128.3,128.1,128.1,125.7,52.8$, $39.9,30.9,22.6,-2.4$.

IR (neat) 2961, 1624, 1248, 1108, 814, 778, 735, $697 \mathrm{~cm}^{-1}$.

HRMS (El) Calcd. for $\mathrm{C}_{20} \mathrm{H}_{26} \mathrm{OSi}\left([\mathrm{M}]^{+}\right)$: 310.1748; Found: 310.1748 .<smiles>CC(C)(Cc1ccccc1)C(=O)Oc1ccccc1</smiles>

1-(Dimethyl(phenyl)silyl)-2,2-dimethyl-3-phenylpropan-1-one (2w). The title compound was synthesized according to general procedure using (2-bromo-2-methylpropyl)benzene (43 mg, $0.2 \mathrm{mmol}$ ). 
The crude material was purified by silica gel chromatography (hexane /ethyl acetate $=20: 1$ ) to afford the title compound as a colorless oil ( $58 \mathrm{mg}, 98 \%$ yield).

1H NMR (500 MHz, $\left.\mathrm{CDCl}_{3}\right) \delta 7.56-7.55(\mathrm{~m}, 2 \mathrm{H}), 7.43-7.34(\mathrm{~m}, 3 \mathrm{H}), 7.25-7.16(\mathrm{~m}, 3 \mathrm{H}), 7.01-$ $6.95(\mathrm{~m}, 2 \mathrm{H}), 2.70(\mathrm{~s}, 2 \mathrm{H}), 0.97(\mathrm{~s}, 6 \mathrm{H}), 0.53(\mathrm{~s}, 6 \mathrm{H})$.

${ }^{13} \mathrm{C} \mathrm{NMR}\left(126 \mathrm{MHz}, \mathrm{CDCl}_{3}\right) \delta 248.3,137.7,135.9,134.1,130.5,129.6,128.0,127.8,126.1,53.3$, 43.1, 22.4, -2.5.

IR (neat) 2963, 1629, 1248, 1108, 809, 779, 735, $700 \mathrm{~cm}^{-1}$.

HRMS (El) Calcd. for $\mathrm{C}_{19} \mathrm{H}_{24} \mathrm{OSi}([\mathrm{M}]+)$ : 296.1591; Found: 296.1601.<smiles>CCC(CC)(CC)C(=O)Oc1ccccc1</smiles>

1-(Dimethyl(phenyl)silyl)-2,2-diethylbutan-1-one (2x). The title compound was synthesized according to general procedure using 3-bromo-3-ethylpentane (36 mg, $0.2 \mathrm{mmol}$ ). The crude material was purified by silica gel chromatography (hexane /ethyl acetate $=20: 1$ ) to afford the title compound as a colorless oil (50 mg, 96\% yield).

${ }^{1} \mathrm{H}$ NMR $\left(500 \mathrm{MHz}, \mathrm{CDCl}_{3}\right) \delta$ 7.60-7.58 (m, 2H), $7.40-7.33(\mathrm{~m}, 3 \mathrm{H}), 1.48(\mathrm{q}, J=7.5 \mathrm{~Hz}, 6 \mathrm{H}), 0.57$ (t, $J=7.5 \mathrm{~Hz}, 9 \mathrm{H}), 0.52(\mathrm{~d}, J=0.5 \mathrm{~Hz}, 6 \mathrm{H})$.

${ }^{13} \mathrm{C}$ NMR $\left(126 \mathrm{MHz}, \mathrm{CDCl}_{3}\right) \delta$ 250.2, 136.1, 134.1, 129.5, 127.9, 60.0, 23.4, 8.0, -2.4.

IR (neat) 2964, 2878, 1694, 1626, 1247, 1108, 805, 776, $700 \mathrm{~cm}^{-1}$.

HRMS (El) Calcd. for $\mathrm{C}_{16} \mathrm{H}_{26} \mathrm{OSi}\left([\mathrm{M}]^{+}\right)$: 262.1748; Found: 262.1752.

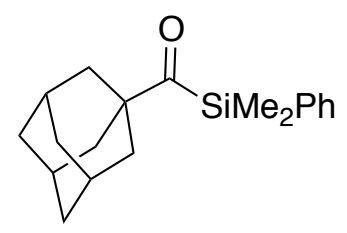

((1S,3s)-Adamantan-1-yl)(dimethyl(phenyl)silyl)methanone (2y). The title compound was synthesized according to general procedure using 1-bromoadamantane (43 mg, $0.2 \mathrm{mmol}$ ). The crude material was purified by silica gel chromatography (hexane lethyl acetate $=20: 1$ ) to afford the title compound as a colorless oil (44 mg, 73\% yield).

${ }^{1} \mathbf{H}$ NMR $\left(500 \mathrm{MHz}, \mathrm{CDCl}_{3}\right) \delta 7.56-7.54(\mathrm{~m}, 2 \mathrm{H}), 7.42-7.34(\mathrm{~m}, 3 \mathrm{H}), 1.96$ (brs, 3H), $1.70-1.67$ $(\mathrm{m}, 9 \mathrm{H}), 1.59-1.56(\mathrm{~m}, 3 \mathrm{H}), 0.54(\mathrm{~s}, 6 \mathrm{H})$.

${ }^{13} \mathrm{C}$ NMR $\left(126 \mathrm{MHz}, \mathrm{CDCl}_{3}\right) \delta 247.9,136.2,134.1,129.5,128.0,52.2,36.7,36.6,27.8,-2.3$.

IR (neat) 2901, 2848, 1623, 1247, 1108, 827, 792, 733, 700, $647 \mathrm{~cm}^{-1}$.

HRMS (ESI) Calcd. for $\mathrm{C}_{19} \mathrm{H}_{26} \mathrm{ONaSi}\left([\mathrm{M}+\mathrm{Na}]^{+}\right)$: 321.1646; Found: 321.1648 . 


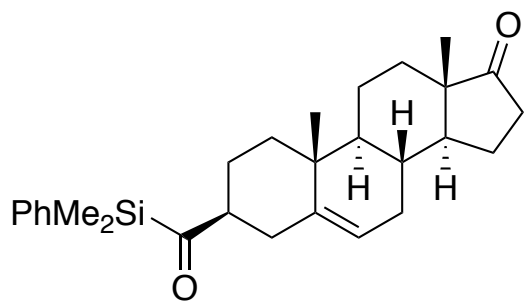

Compound 5. The title compound was synthesized according to general procedure using $3 \beta$ iododandrost-5-en-17-one ( $80 \mathrm{mg}, 0.2 \mathrm{mmol}$ ). The crude material was purified by silica gel chromatography (hexane /ethyl acetate $=6: 1$ ) to afford a mixture of two diastereomeric isomers as a white solid (76 mg, $88 \%$ yield). The $d r$ was determined to be $4: 1$ by LC-MS. The assignment of $3 \beta$ configuration in the major isomer was based on the large coupling constant at $2.71 \mathrm{ppm}$, typical of an axial proton in a cyclohexane chair system.

${ }^{1} \mathrm{H}$ NMR $\left(500 \mathrm{MHz}, \mathrm{CDCl}_{3}\right) \delta 7.55-7.54(\mathrm{~m}, 2 \mathrm{H}), 7.42-7.34(\mathrm{~m}, 3 \mathrm{H}), 5.24(\mathrm{~d}, J=5.0 \mathrm{~Hz}, 1 \mathrm{H}), 2.71$ (tt, $J=12.4,3.4 \mathrm{~Hz}, 1 \mathrm{H}), 2.47-2.41(\mathrm{~m}, 1 \mathrm{H}), 2.22-2.17(\mathrm{t}, J=7.1 \mathrm{~Hz}, 1 \mathrm{H}), 2.12-2.02(\mathrm{~m}, 2 \mathrm{H}), 1.96-$ $1.89(\mathrm{~m}, 2 \mathrm{H}), 1.87-1.79(\mathrm{~m}, 2 \mathrm{H}), 163-1.57(\mathrm{~m}, 4 \mathrm{H}), 1.54-1.30(\mathrm{~m}, 3 \mathrm{H}), 1.28-1.21(\mathrm{~m}, 2 \mathrm{H}), 1.03-0.96$ $(\mathrm{m}, 2 \mathrm{H}), 0.91(\mathrm{~s}, 3 \mathrm{H}), 0.85(\mathrm{~s}, 3 \mathrm{H}), 0.49(\mathrm{~s}, 3 \mathrm{H}), 0.49(\mathrm{~s}, 3 \mathrm{H})$.

${ }^{13} \mathrm{C}$ NMR $\left(126 \mathrm{MHz}, \mathrm{CDCl}_{3}\right) \delta 248.0,221.1,141.9,134.7,133.9,129.8,128.1,119.7,56.8,51.7$, $50.3,47.4,38.3,37.2,35.8,32.7,31.4,31.3,30.7,23.2,21.8,20.1,19.2,13.5,-4.3,-4.4$.

IR (neat) 2911, 2833, 1735, 1626, 1249, 804, 781, $696 \mathrm{~cm}^{-1}$.

HRMS (ESI) Calcd. for $\mathrm{C}_{28} \mathrm{H}_{39} \mathrm{O}_{2} \mathrm{Si}([\mathrm{M}+\mathrm{H}]+)$ ): 435.2714; Found: 435.2717.

\section{Preparation of Alkyl lodides Substrates}

1-iodooctane (1a) was purchased from Alfa Aesar. 1-lodo-3-phenylpropane (1b), 1-chloro-6iodohexane (1d), 2-iodopropane (10), $t$-butyl bromide (1u) and 1-bromoadamantane (1y) were purchased from Sigma-Aldrich. lodocyclopentane (1q), 4-iodo-tetrahydropyran (1s) and N-Boc-4-iodopiperidine (1t) were purchased from Fischer Scientific. lodocyclohexane (1r) and 6-lodohexene (8) were purchased from TCl America.<smiles>CC(C)(CCI)COBr</smiles>

(((6-lodohexyl)oxy)methyl)benzene (1c) was prepared according to the literature and has been previously characterized. 6<smiles>ICCOc1ccco1</smiles>

2-(((6-lodohexyl)oxy)methyl)furan (1e) was prepared according to the literature and has been previously characterized. 6<smiles>IC[13CH2]C[13CH2]OCCc1cccs1</smiles> 
2-(2-((6-iodohexyl)oxy)ethyl)thiophene (1f) was prepared according to the literature and has been previously characterized. 6<smiles>CC(C)(CI)Cn1ccc2ccccc21</smiles>

1-(6-lodohexyl)-1H-indole (1g) was prepared according to the literature and has been previously characterized. 6<smiles>CC(CI)COc1ccc(C(F)(F)F)cc1</smiles>

1-((6-lodohexyl)oxy)-4-(trifluoromethyl)benzene (1h) was prepared according to the known literature and has been previously characterized. ${ }^{7}$<smiles>COC(=O)c1ccc(OCC(C)CI)cc1</smiles>

Methyl 4-((6-iodohexyl)oxy)benzoate (1i) was prepared according to the known literature and has been previously characterized. 8<smiles>CC(CI)COc1ccc(C#N)cc1</smiles>

4-((6-lodohexyl)oxy)benzonitrile (1j) was prepared according to the known literature and has been previously characterized. ${ }^{7}$<smiles>CC(C)(CI)COc1ccc(Cl)cc1</smiles>

1-Chloro-4-((6-iodohexyl)oxy)benzene (1k) was prepared according to the known literature and has been previously characterized. ${ }^{6}$<smiles>CC(CI)COc1ccc(Br)cc1</smiles>

1-Bromo-4-((6-iodohexyl)oxy)benzene (1I) was prepared according to the known literature and has been previously characterized. ${ }^{9}$<smiles>CC(CCCI)COc1ccc(I)cc1</smiles>

1-lodo-4-((6-iodohexyl)oxy)benzene (1m) was prepared according to the known literature and has been previously characterized. ${ }^{7}$ 


$$
W_{7}=
$$

10-lodo-1-decene (1n) was prepared according to the known literature and has been previously characterized. 10<smiles>CC(I)C1CCCCC1</smiles>

1-Cyclohexyliodoethane (1p) was prepared according to the known literature and has been previously characterized. ${ }^{11}$<smiles>CC(C)(Br)CCc1ccccc1</smiles>

(3-Bromo-3-methylbutyl)benzene (1v) was prepared according to the literature and has been previously characterized. ${ }^{12}$<smiles>CC(C)(Br)Cc1ccccc1</smiles>

(2-Bromo-2-methylpropyl)benzene (1w) was prepared according to the literature and has been previously characterized. ${ }^{13}$<smiles>CCC(Br)(Br)CC</smiles>

3-Bromo-3-ethylpentane (1x) was prepared according to the known literature and has been previously characterized. ${ }^{14}$<smiles>C[C@]12CC[C@H]3[C@H](CC=C4C[C@@H](I)CC[C@@]43C)[C@@H]1CCC2=O</smiles>

3ß-iododandrost-5-en-17-one (4) was prepared according to the known literature and has been previously characterized..$^{15}$

\section{Use of other Bpin-SiR 3 reagents}

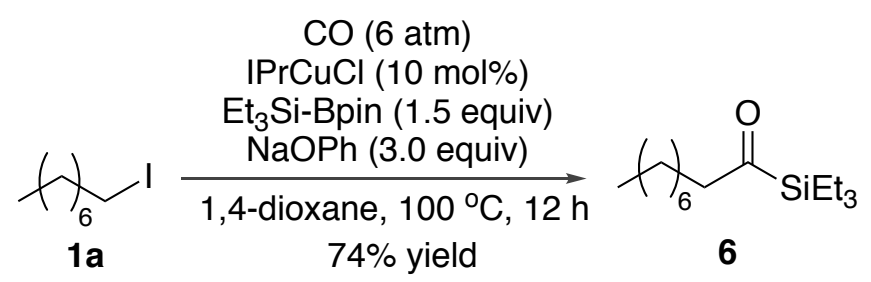

The reaction was performed according to the general procedure for synthesis of acylsilanes using 1iodooctane $(48 \mathrm{mg}, 0.2 \mathrm{mmol})$ and $\mathrm{Et}_{3} \mathrm{Si}-\mathrm{Bpin}(73 \mathrm{mg}, 0.3 \mathrm{mmol})$. The reaction was conducted at $100^{\circ} \mathrm{C}$ for $12 \mathrm{~h}$. The crude material was purified by silica gel chromatography (hexane /ethyl acetate $=20: 1$ ) to afford 
an inseparable mixture of acylsilane 6 and remaining Bpin-SiEt $t_{3}$ as a colorless oil (56 mg). The ratio of the 6 and Bpin-SiEt ${ }_{3}$ was determined to be $2: 1$ by the ${ }^{1} \mathrm{HNMR}$ analysis of the isolated mixture.

$1 \mathrm{H}$ NMR (400 MHz, $\left.\mathrm{CDCl}_{3}\right) \delta 2.54(\mathrm{t}, J=7.3 \mathrm{~Hz}, 2 \mathrm{H}), 1.53-1.46(\mathrm{~m}, 2 \mathrm{H}), 1.25$ (brs, 10H), 0.96 (t, $J$ $=7.9 \mathrm{~Hz}, 9 \mathrm{H}), 0.87(\mathrm{t}, J=6.9 \mathrm{~Hz}, 3 \mathrm{H}), 0.72(\mathrm{q}, J=7.9 \mathrm{~Hz}, 6 \mathrm{H})$.

${ }^{13} \mathrm{C}$ NMR $\left(101 \mathrm{MHz}, \mathrm{CDCl}_{3}\right) \delta 248.4,50.1,31.8,29.5,29.4,29.1,22.6,21.9,14.0,7.2,2.2$.

IR (neat) 2953, 2923, 2874, 2854, 1640, 1462, 1016, $720 \mathrm{~cm}^{-1}$.

HRMS (ESI) Calcd. for $\mathrm{C}_{15} \mathrm{H}_{33} \mathrm{OSi}([\mathrm{M}+\mathrm{H}]+)$ : 257.2295; Found: 257.2288 .

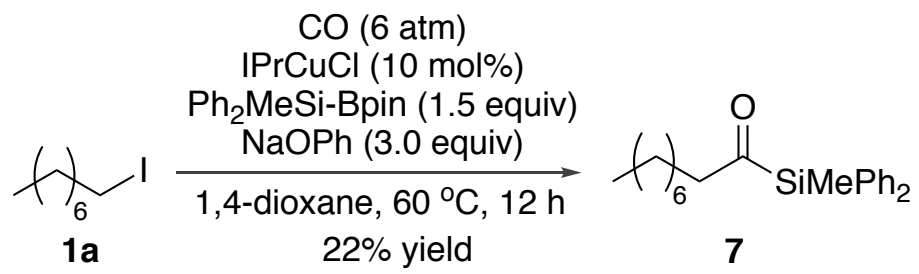

The reaction was performed according to the general procedure for synthesis of acylsilanes using 1iodooctane (48 mg, $0.2 \mathrm{mmol}$ ) and $\mathrm{Ph}_{2} \mathrm{MeSi}-B p i n(97 \mathrm{mg}, 0.3 \mathrm{mmol}$ ). The crude material was purified by silica gel chromatography (hexane /toluene $=3: 1$ ) to afford the title compound as a colorless oil ( $15 \mathrm{mg}$, $22 \%$ yield).

${ }^{1} \mathrm{H}$ NMR (400 MHz, $\left.\mathrm{CDCl}_{3}\right) \delta 7.60-7.58(\mathrm{~m}, 4 \mathrm{H}), 7.46-7.38(\mathrm{~m}, 6 \mathrm{H}), 2.64(\mathrm{t}, J=7.2 \mathrm{~Hz}, 2 \mathrm{H}), 1.50$ $-1.41(\mathrm{~m}, 2 \mathrm{H}), 1.26-1.17(\mathrm{~m}, 10 \mathrm{H}), 0.86(\mathrm{t}, J=7.0 \mathrm{~Hz}, 3 \mathrm{H}), 0.75(\mathrm{~s}, 3 \mathrm{H})$.

${ }^{13} \mathrm{C}$ NMR $\left(101 \mathrm{MHz}, \mathrm{CDCl}_{3}\right) \delta 244.8,135.0,132.9,130.0,128.2,49.8,31.8,29.3,29.2,29.1,22.6$, 22.2, 14.1, -5.3.

IR (neat) 2923, 2853, 1642, 1428, 1112, 792, 729, $698 \mathrm{~cm}^{-1}$.

HRMS (ESI) Calcd. for $\mathrm{C}_{22} \mathrm{H}_{31} \mathrm{OSi}\left([\mathrm{M}+\mathrm{H}]^{+}\right)$: 339.2139; Found: 339.2123 .

\section{One-pot synthesis of $\alpha$-hydroxylsilanes}

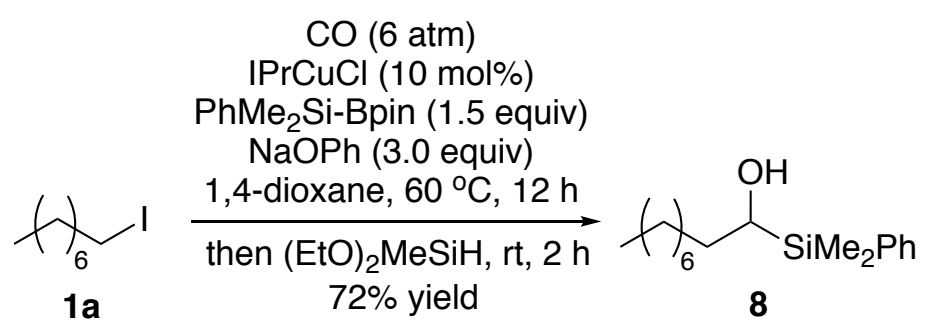

In a glovebox, NaOPh (35 mg, $0.3 \mathrm{mmol}, 3.0$ equiv), IPrCuCl (5 mg, $0.01 \mathrm{mmol}, 10 \mathrm{~mol} \%$ ) and 1,4dioxane $(6.0 \mathrm{~mL})$ were added to a $20-\mathrm{mL}$ vial with a $1.5 \mathrm{~cm}$ stir bar. The mixture was stirred at room temperature for $2 \mathrm{~min}$ before $\mathrm{PhMe}_{2} \mathrm{Si}$-Bpin ( $40 \mu \mathrm{L}, 0.15 \mathrm{mmol}, 1.5$ equiv) and 1 -iodooctane (24 mg, 0.1 $\mathrm{mmol}$ ) were added sequentially at room temperature. The vial was then placed in an aluminum rack within a Parr pressure reactor. Next, the reactor was closed and taken out of the glovebox. A CO gas cylinder with a pressure regulator was connected to the reactor. The reactor was pressurized with $\mathrm{CO}$ gas to 6 atm and released after $5 \mathrm{~min}$. This procedure was repeated three times, after which the reaction was stirred at $60{ }^{\circ} \mathrm{C}$ for $12 \mathrm{~h}$. Then, the reaction was cooled to room temperature and the $\mathrm{CO}$ gas was released. The 
reactor was brought into the glovebox and $(\mathrm{EtO})_{2} \mathrm{MeSiH}(26.8 \mathrm{mg}, 0.2 \mathrm{mmol})$ was added to the reaction mixture at room temperature. After the reaction was stirred at room temperature for $2 \mathrm{~h}$, the vial was taken out of the glovebox. Next, $\mathrm{HCl}(1 \mathrm{~N}, 10 \mathrm{~mL})$ was added to reaction mixture and the reaction was stirred vigorously at room temperature for $10 \mathrm{~min}$. Then, the reaction mixture was diluted with $\mathrm{Et}_{2} \mathrm{O}(20 \mathrm{~mL})$. The organic phase was separated and the aqueous layer was extracted twice with $\mathrm{Et}_{2} \mathrm{O}(2 \times 20 \mathrm{~mL})$. The combined organic layers were washed with brine, dried over $\mathrm{MgSO}_{4}$, filtered and concentrated on a rotary evaporator. The crude material was purified by silica gel chromatography (hexane /ethyl acetate $=10: 1$ ) to afford the title compound as a colorless oil $(20 \mathrm{mg}, 72 \%$ yield).

1H NMR (500 MHz, $\left.\mathrm{CDCl}_{3}\right) \delta 7.59-7.55(\mathrm{~m}, 2 \mathrm{H}), 7.39-7.37(\mathrm{~m}, 3 \mathrm{H}), 3.53-3.50(\mathrm{~m}, 1 \mathrm{H}), 1.56-$ $1.50(\mathrm{~m}, 3 \mathrm{H}), 1.33-1.23(\mathrm{~m}, 12 \mathrm{H}), 0.88(\mathrm{t}, J=6.9 \mathrm{~Hz}, 3 \mathrm{H}), 0.35(\mathrm{~s}, 3 \mathrm{H}), 0.34(\mathrm{~s}, 3 \mathrm{H})$.

${ }^{13} \mathrm{C} \mathrm{NMR}\left(126 \mathrm{MHz}, \mathrm{CDCl}_{3}\right) \delta 136.8,134.1,129.3,127.9,65.5,33.4,31.8,29.6,29.5,29.3,26.8$, 22.6, $14.1,-5.4,-5.7$.

IR (neat) 3420, 2923, 2852, 1246, 1112, 812, 775, 733, $699 \mathrm{~cm}^{-1}$.

HRMS (ESI) Calcd. for $\mathrm{C}_{23} \mathrm{H}_{31} \mathrm{OSi}\left([\mathrm{M}+\mathrm{H}]^{+}\right)$: 279.2139; Found: 279.2138 .

\section{Mechanistic studies}

\subsection{Radical trapping experiments}

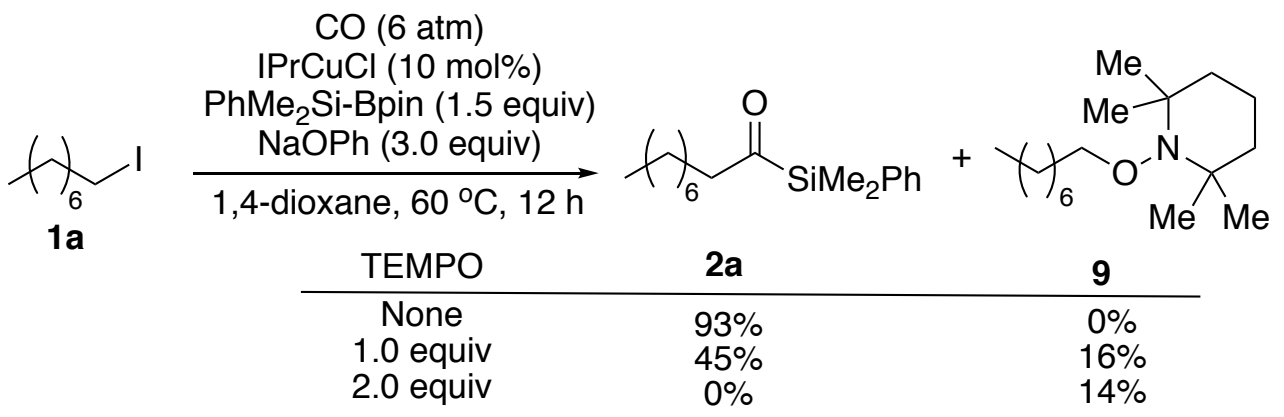

In a glovebox, $\mathrm{NaOPh}$ (35 mg, $0.3 \mathrm{mmol}, 3.0$ equiv), IPrCuCl (5 mg, $0.01 \mathrm{mmol}, 10 \mathrm{~mol} \%$ ) and 1,4dioxane $(6.0 \mathrm{~mL})$ were added to a $20-\mathrm{mL}$ vial with a $1.5 \mathrm{~cm}$ stir bar. The mixture was stirred at room temperature for $2 \mathrm{~min}$ before $\mathrm{PhMe}_{2} \mathrm{Si}$-Bpin ( $40 \mu \mathrm{L}, 0.15 \mathrm{mmol}, 1.5$ equiv), 1 -iodooctane (24 mg, $0.1 \mathrm{mmol}$ ) and TEMPO (1.0 or 2.0 equiv) were added sequentially at room temperature. The vial was then placed in an aluminum rack within a Parr pressure reactor. Next, the reactor was closed and taken out of the glovebox. A CO gas cylinder with a pressure regulator was connected to the reactor. The reactor was pressurized with $\mathrm{CO}$ gas to $6 \mathrm{~atm}$ and released after $5 \mathrm{~min}$. This procedure was repeated three times, after which the reaction was stirred at $60{ }^{\circ} \mathrm{C}$ for $12 \mathrm{~h}$. Then, the reaction was cooled to room temperature and the $\mathrm{CO}$ gas was released. The solvent was removed under reduced pressure. The residue was diluted with $\mathrm{Et}_{2} \mathrm{O}$ and filtered through a pad of silica gel (a pipette with about $5 \mathrm{~cm}$ silica gel). The filtrate was concentrated under reduced pressure. The residue was dissolved in $\mathrm{CDCl}_{3}$ and $\mathrm{CH}_{2} \mathrm{Br}_{2}$ (0.1 mmol) was added as internal standard for ${ }^{1} \mathrm{H}$ NMR analysis to determine the yields of the acylsilane $2 \mathrm{a}$ and the radical trapping product $9 .{ }^{16}$ 
7.2 Radical clock experiments

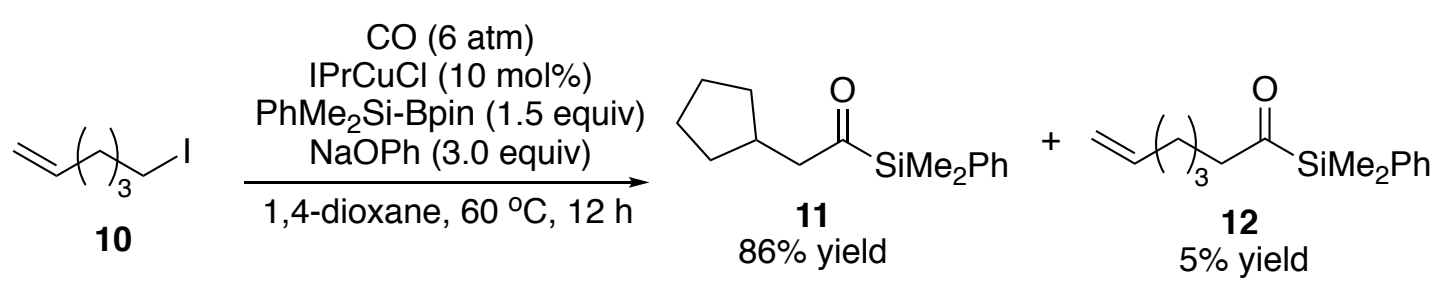

The reaction was performed according to the general procedure for synthesis of acylsilanes using 6iodohexene (42 mg, $0.2 \mathrm{mmol}$ ). The crude material was purified by silica gel chromatography (hexane /ethyl acetate $=20: 1$ ) to afford a mixture of ring-closing acylsilane 11 and the compound 12as a colorless oil (43 $\mathrm{mg}, 91 \%$ yield). The ratio of the 11 and 12 was determined to be $17: 1$ by the ${ }^{1} \mathrm{HNMR}$ analysis of the isolated mixture.

1H NMR (500 MHz, $\left.\mathrm{CDCl}_{3}\right)$ ס 7.56-7.54 (m, 2H), $7.43-7.35(\mathrm{~m}, 3 \mathrm{H}), 2.60(\mathrm{~d}, J=6.9 \mathrm{~Hz}, 2 \mathrm{H}), 2.24$ $-2.15(\mathrm{~m}, 1 \mathrm{H}), 1.69(\mathrm{dt}, J=11.2,6.4 \mathrm{~Hz}, 2 \mathrm{H}), 1.56-1.42(\mathrm{~m}, 4 \mathrm{H}), 0.97-0.90(\mathrm{~m}, 2 \mathrm{H}), 0.48(\mathrm{~s}, 6 \mathrm{H})$.

${ }^{13} \mathrm{C}$ NMR $\left(126 \mathrm{MHz}, \mathrm{CDCl}_{3}\right) \delta 246.7,134.6,133.9,129.8,128.1,55.1,33.9,32.6,24.9,-4.8$.

IR (neat) 2950, 2866, 1642, 1248, 1110, 834, 815, 778, 733, $698 \mathrm{~cm}^{-1}$.

HRMS (ESI) Calcd. for $\mathrm{C}_{15} \mathrm{H}_{22} \mathrm{ONaSi}\left([\mathrm{M}+\mathrm{Na}]^{+}\right)$: 269.1333; Found: 269.1335.

7.3 Stoichiometric coupling of IPrCu-SiMe ${ }_{2} \mathrm{Ph}$ with alkyl iodides under $\mathrm{CO}$

\begin{tabular}{|c|c|c|c|c|}
\hline $\begin{array}{l}\text { IPrCuOtBu } \\
\text { (1.0 equiv) }\end{array}$ & + & $\begin{array}{c}\mathrm{PhMe}_{2} \text { Si-Bpin } \\
\text { (1.0 equiv) }\end{array}$ & $\overrightarrow{\text { toluene, } \mathrm{rt}, 1 \mathrm{~h}}$ & $\begin{array}{c}\text { IPrCu}-\mathrm{SiMe}_{2} \mathrm{Ph} \\
\mathbf{1 3}\end{array}$ \\
\hline
\end{tabular}

The IPrCu-SiMe $\mathrm{P}_{2} \mathrm{Ph}$ was prepared according to the previous literature. ${ }^{17}$ In a glovebox, IPrCuOtBu (262 mg, $0.5 \mathrm{mmol}, 1.0$ equiv) and toluene $(5 \mathrm{~mL}$ ) were added to a $20-\mathrm{mL}$ vial with a $1.5 \mathrm{~cm}$ stir bar. Then $\mathrm{PhMe}_{2} \mathrm{Si}$-Bpin (131 mg, $0.5 \mathrm{mmol}, 1.0$ equiv) was dissolved in toluene (1.0 mL) and added to the above mixture at room temperature. The reaction was stirred at room temperature for $1 \mathrm{~h}$. Then, the solvent was removed under reduced pressure and the solid was washed with pentane to afford an off-white solid (205 $\mathrm{mg}, 70 \%$ yield).

${ }^{1} \mathrm{H}$ NMR $\left(500 \mathrm{MHz}, \mathrm{C}_{6} \mathrm{D}_{6}\right) \delta 7.48-7.47(\mathrm{~m}, 2 \mathrm{H}), 7.25-7.21(\mathrm{~m}, 5 \mathrm{H}), 7.07(\mathrm{~d}, J=7.7 \mathrm{~Hz}, 4 \mathrm{H}), 6.22$ (s, 2H), $2.66-2.50(\mathrm{~m}, 4 \mathrm{H}), 1.36(\mathrm{~d}, J=6.8 \mathrm{~Hz}, 12 \mathrm{H}), 1.08(\mathrm{~d}, J=6.9 \mathrm{~Hz}, 12 \mathrm{H}), 0.43(\mathrm{~s}, 6 \mathrm{H})$.

${ }^{13} \mathrm{C}$ NMR $\left(126 \mathrm{MHz}, \mathrm{C}_{6} \mathrm{D}_{6}\right) \delta 185.3,153.3,145.8,135.2,135.0,130.5,127.1,125.9,124.2,122.0$, 29.0, 25.2, 23.6, 4.3 .

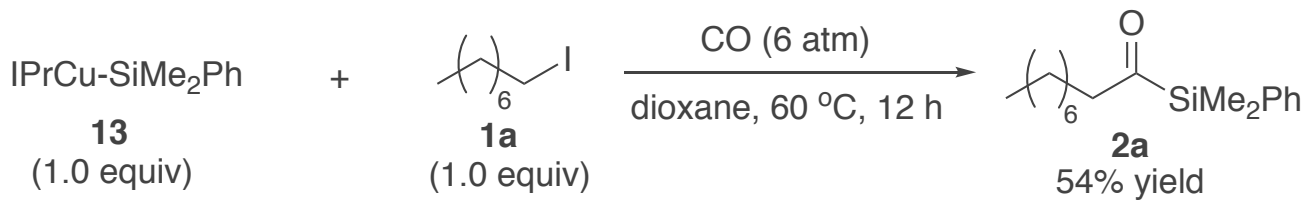

In a glovebox, IPrCu-SiMe 2 Ph (29 mg, $0.05 \mathrm{mmol})$ and THF $(4 \mathrm{~mL})$ were added to a $20-\mathrm{mL}$ vial with a $1.5 \mathrm{~cm}$ stir bar. Then 1-iodooctane $(12 \mathrm{mg}, 0.05 \mathrm{mmol})$ was dissolved in THF $(1.0 \mathrm{~mL})$ and added to the above solution at room temperature. The vial was placed in an aluminum rack within a Parr pressure 
reactor. Next, the reactor was closed and taken out of the glovebox. A CO gas cylinder with a pressure regulator was connected to the reactor. The reactor was pressurized with $\mathrm{CO}$ gas to $6 \mathrm{~atm}$ and released after $5 \mathrm{~min}$. This procedure was repeated three times, after which the reaction heated to $60 \circ \mathrm{C}$ for $12 \mathrm{~h}$. Then, the reaction was cooled to room temperature and the $\mathrm{CO}$ gas was released. The solvent was removed under reduced pressure. The residue was diluted with $\mathrm{Et}_{2} \mathrm{O}$ and filtered through a pad of silica gel (a pipette with about $5 \mathrm{~cm}$ silica gel). The filtrate was concentrated under reduced pressure. The residue was dissolved in $\mathrm{CDCl}_{3}$ and $\mathrm{CH}_{2} \mathrm{Br}_{2}(0.1 \mathrm{mmol})$ was added as internal standard for ${ }^{1} \mathrm{H}$ NMR analysis. The ${ }^{1} \mathrm{H}$ NMR analysis showed that the compound $2 \mathrm{a}$ was obtained in $54 \%$ yield.

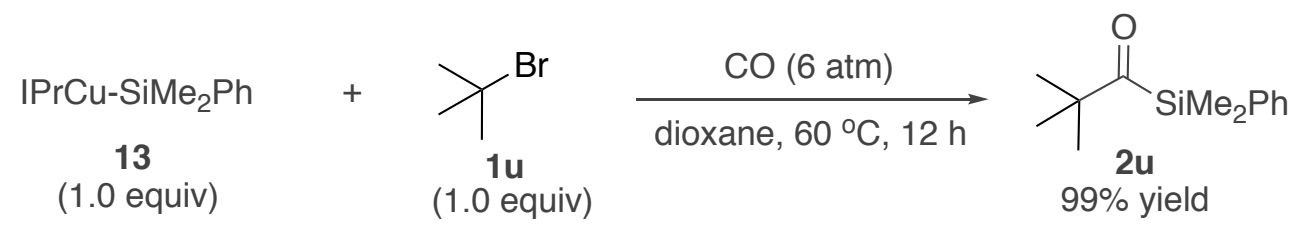

In a glovebox, IPrCu-SiMe 2 Ph $(29 \mathrm{mg}, 0.05 \mathrm{mmol})$ and THF $(4 \mathrm{~mL})$ were added to a $20-\mathrm{mL}$ vial with a $1.5 \mathrm{~cm}$ stir bar. Then $t$-butyl bromide $(7 \mathrm{mg}, 0.05 \mathrm{mmol})$ was dissolved in THF $(1.0 \mathrm{~mL})$ and added to the above solution at room temperature. The vial was placed in an aluminum rack within a Parr pressure reactor. Next, the reactor was closed and taken out of the glovebox. A CO gas cylinder with a pressure regulator was connected to the reactor. The reactor was pressurized with $\mathrm{CO}$ gas to $6 \mathrm{~atm}$ and released after $5 \mathrm{~min}$. This procedure was repeated three times, after which the reaction heated to $60{ }^{\circ} \mathrm{C}$ for $12 \mathrm{~h}$. Then, the reaction was cooled to room temperature and the $\mathrm{CO}$ gas was released. The solvent was removed under reduced pressure. The residue was diluted with $\mathrm{Et}_{2} \mathrm{O}$ and filtered through a pad of silica gel (a pipette with about $5 \mathrm{~cm}$ silica gel). The filtrate was concentrated under reduced pressure. The residue was dissolved in $\mathrm{CDCl}_{3}$ and $\mathrm{CH}_{2} \mathrm{Br}_{2}(0.1 \mathrm{mmol})$ was added as internal standard for ${ }^{1} \mathrm{H} \mathrm{NMR}$ analysis. The ${ }^{1} \mathrm{H}$ NMR analysis showed that the compound $2 \mathrm{u}$ was obtained in $99 \%$ yield.

\subsection{Competition experiments}

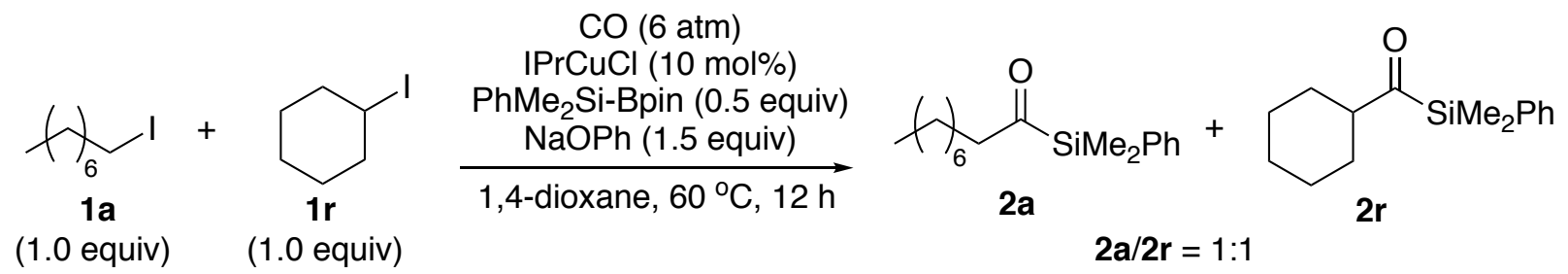

In a glovebox, NaOPh (17 mg, $0.15 \mathrm{mmol}, 1.5$ equiv), IPrCuCl (5 mg, $0.01 \mathrm{mmol}, 10 \mathrm{~mol} \%$ ) and 1,4dioxane $(6.0 \mathrm{~mL})$ were added to a $20-\mathrm{mL}$ vial with a $1.5 \mathrm{~cm}$ stir bar. The mixture was stirred at room temperature for $2 \mathrm{~min}$ before PhMe 2 Si-Bpin (13 mg, $0.05 \mathrm{mmol}, 0.5$ equiv), 1-iodooctane (1a) (24 mg, 0.1 mmol, 1.0 equiv) and iodocyclohexane (1r) $(21 \mathrm{mg}, 0.1 \mathrm{mmol}, 1.0$ equiv) were added at room temperature. The vial was then placed in an aluminum rack within a Parr pressure reactor. Next, the reactor was closed and taken out of the glovebox. A CO gas cylinder with a pressure regulator was connected to the reactor. The reactor was pressurized with $\mathrm{CO}$ gas to $6 \mathrm{~atm}$ and released after $5 \mathrm{~min}$. This procedure was repeated 
three times, after which the reaction was stirred at $60 \circ \mathrm{C}$ for $12 \mathrm{~h}$. Then, the reaction was cooled to room temperature and the $\mathrm{CO}$ gas was released. The solvent was removed under reduced pressure. The residue was diluted with $\mathrm{Et}_{2} \mathrm{O}$ and filtered through a pad of silica gel (a pipette with about $5 \mathrm{~cm}$ silica gel). The filtrate was concentrated under reduced pressure. The residue was dissolved in $\mathrm{CDCl}_{3}$ and $\mathrm{CH}_{2} \mathrm{Br}_{2}(0.1 \mathrm{mmol})$ was added as internal standard for ${ }^{1} \mathrm{H}$ NMR analysis. The ${ }^{1} \mathrm{H}$ NMR analysis showed that both of $2 \mathrm{a}$ and $2 \mathbf{r}$ were obtained in $24 \%$ yield.

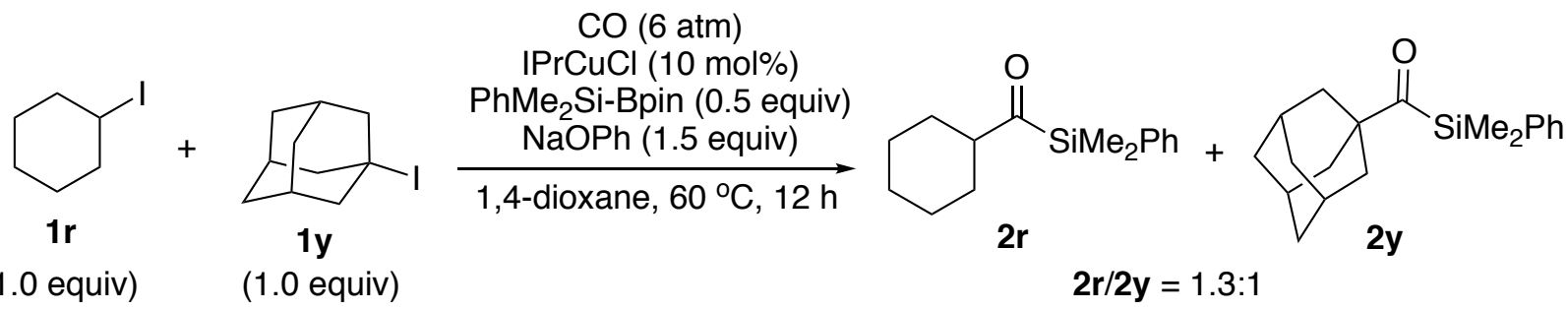

In a glovebox, NaOPh (17 mg, $0.15 \mathrm{mmol}, 1.5$ equiv), IPrCuCl (5 mg, $0.01 \mathrm{mmol}, 10 \mathrm{~mol} \%$ ) and 1,4dioxane $(6.0 \mathrm{~mL})$ were added to a $20-\mathrm{mL}$ vial with a $1.5 \mathrm{~cm}$ stir bar. The mixture was stirred at room temperature for $2 \mathrm{~min}$ before $\mathrm{PhMe}_{2} \mathrm{Si}$-Bpin (13 mg, $0.05 \mathrm{mmol}, 0.5$ equiv), iodocyclohexane (21 mg, 0.1 mmol, 1.0 equiv) and 1-iodoadamantane (26 mg, $0.1 \mathrm{mmol}, 1.0$ equiv) were added at room temperature. The vial was then placed in an aluminum rack within a Parr pressure reactor. Next, the reactor was closed and taken out of the glovebox. A CO gas cylinder with a pressure regulator was connected to the reactor. The reactor was pressurized with $\mathrm{CO}$ gas to $6 \mathrm{~atm}$ and released after $5 \mathrm{~min}$. This procedure was repeated three times, after which the reaction was stirred at $60{ }^{\circ} \mathrm{C}$ for $12 \mathrm{~h}$. Then, the reaction was cooled to room temperature and the $\mathrm{CO}$ gas was released. The solvent was removed under reduced pressure. The residue was diluted with $\mathrm{Et}_{2} \mathrm{O}$ and filtered through a pad of silica gel (a pipette with about $5 \mathrm{~cm}$ silica gel). The filtrate was concentrated under reduced pressure. The residue was dissolved in $\mathrm{CDCl}_{3}$ and $\mathrm{CH}_{2} \mathrm{Br}_{2}(0.1 \mathrm{mmol})$ was added as internal standard for ${ }^{1} \mathrm{H}$ NMR analysis. The ${ }^{1} \mathrm{H}$ NMR analysis showed that $2 \mathrm{r}$ and $2 \mathrm{y}$ were obtained in $25 \%$ and $19 \%$ yields, respectively. 


\section{7. ${ }^{1} \mathrm{H},{ }^{13} \mathrm{C}$ NMR Spectra}
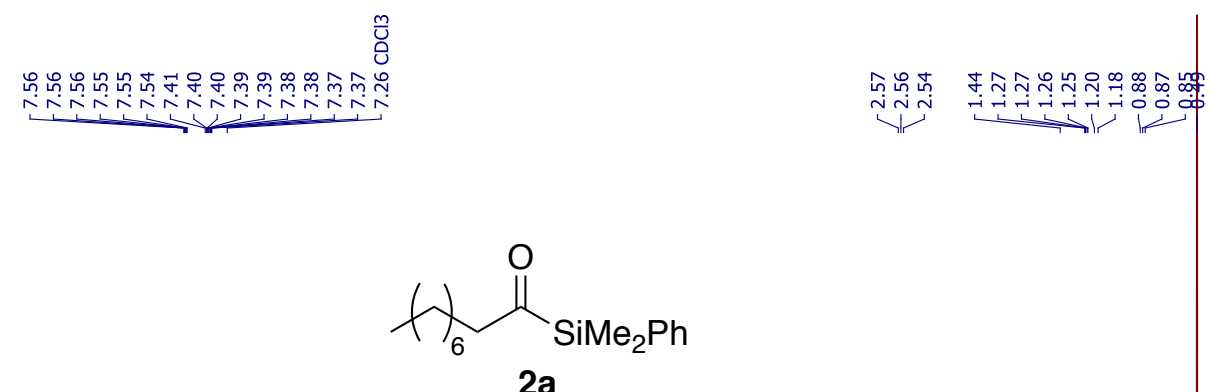

( ${ }^{1} \mathrm{H} \mathrm{NMR}, \mathrm{CDCl}_{3}, 400 \mathrm{MHz}$ )

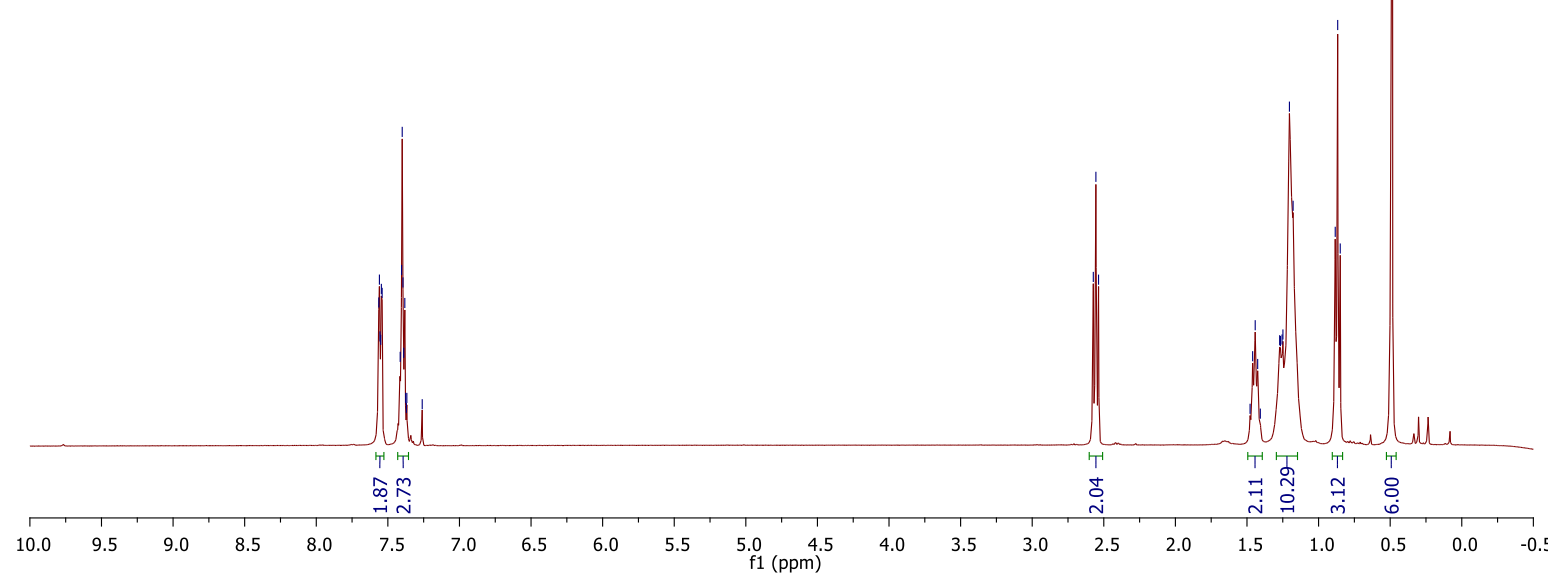

$\stackrel{ }{n}$
$\stackrel{6}{\sigma}$
$\mid$
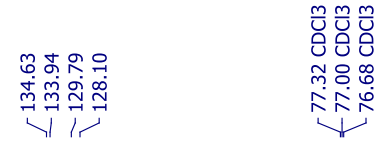

잉

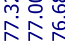

证
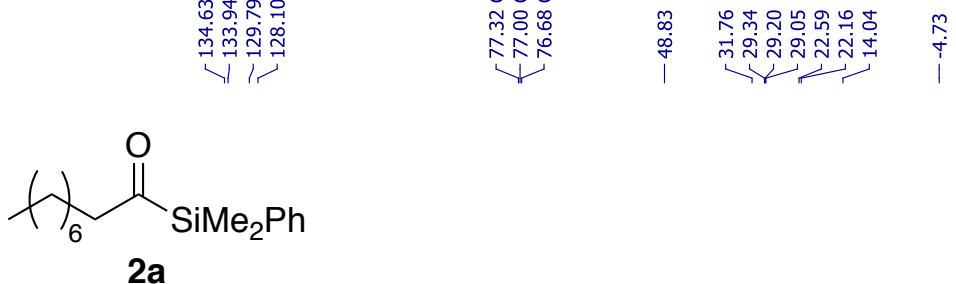

$\left({ }^{13} \mathrm{C} \mathrm{NMR}, \mathrm{CDCl}_{3}, 101 \mathrm{MHz}\right)$

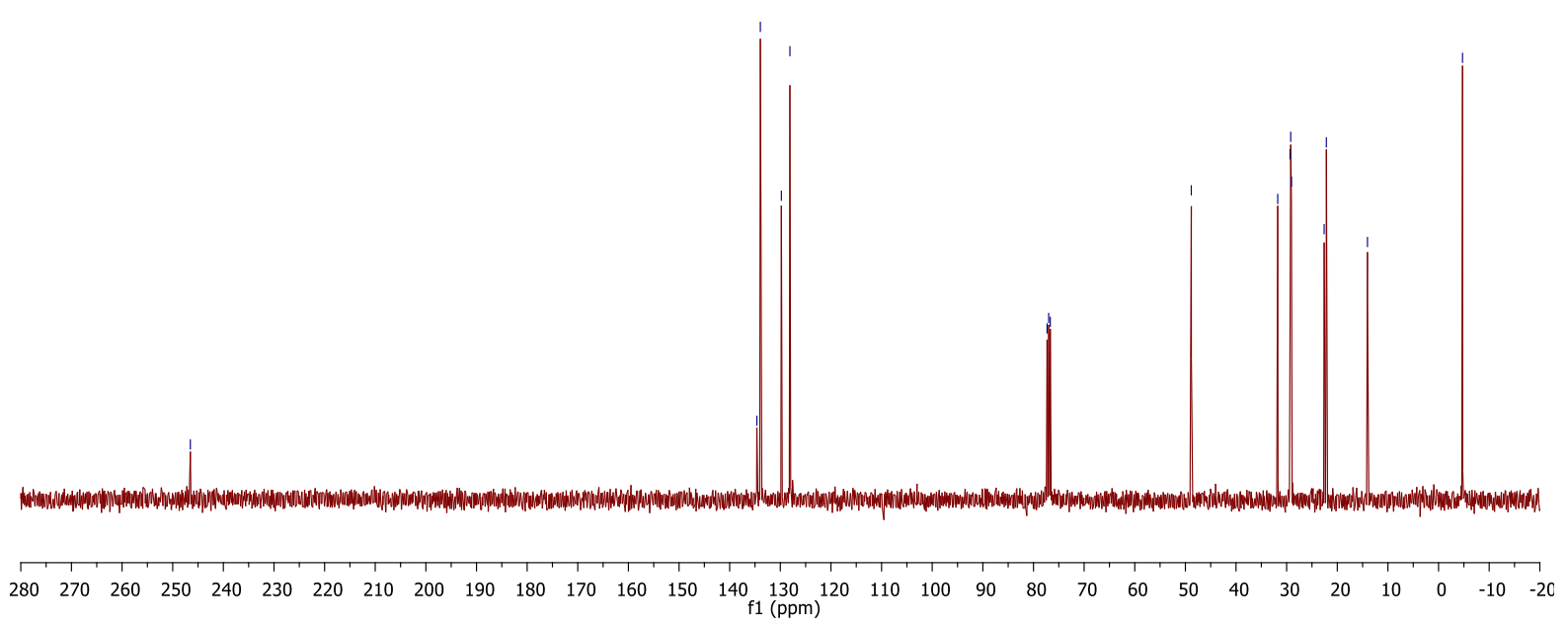




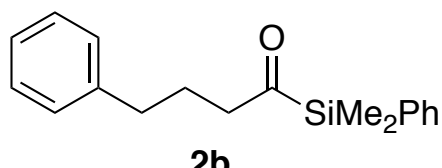

$\left({ }^{1} \mathrm{H} \mathrm{NMR}, \mathrm{CDCl}_{3}, 500 \mathrm{MHz}\right)$

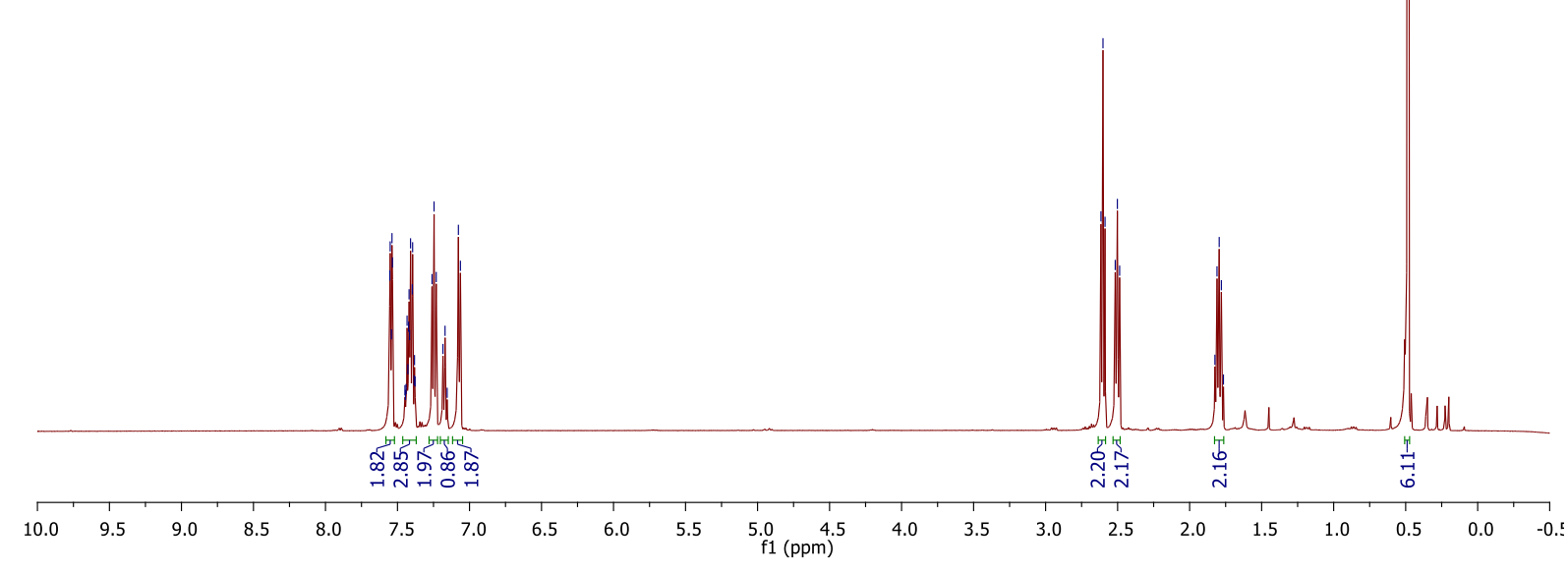

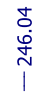

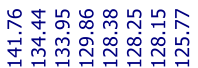

皮

商旁商

이요

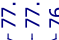

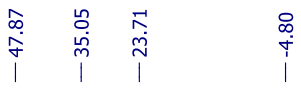<smiles>CSC(=O)CCCc1ccccc1</smiles>

2b

$\left({ }^{13} \mathrm{C}\right.$ NMR, $\left.\mathrm{CDCl}_{3}, 126 \mathrm{MHz}\right)$

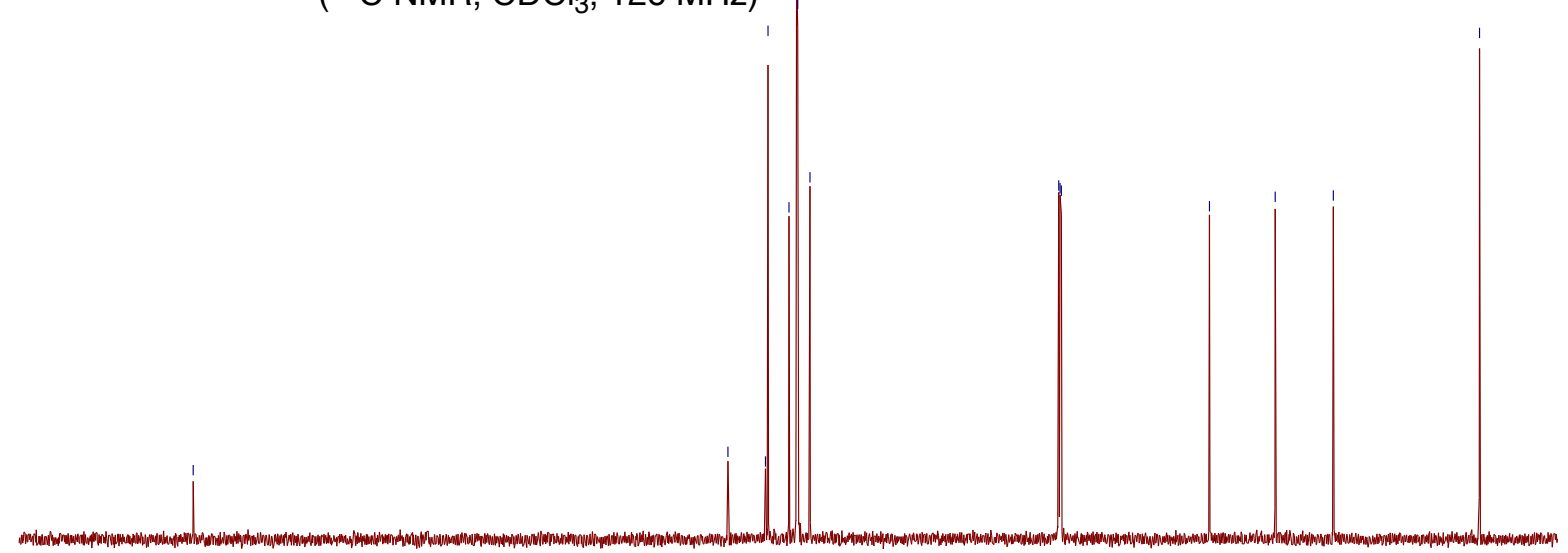

$\begin{array}{llllllllllllllllllllllllllllllllll}280 & 270 & 260 & 250 & 240 & 230 & 220 & 210 & 200 & 190 & 180 & 170 & 160 & 150 & 140 & 130 & 120 & 110 & 100 & 90 & 80 & 70 & 60 & 50 & 40 & 30 & 20 & 10 & 0 & -10 & -20\end{array}$ 


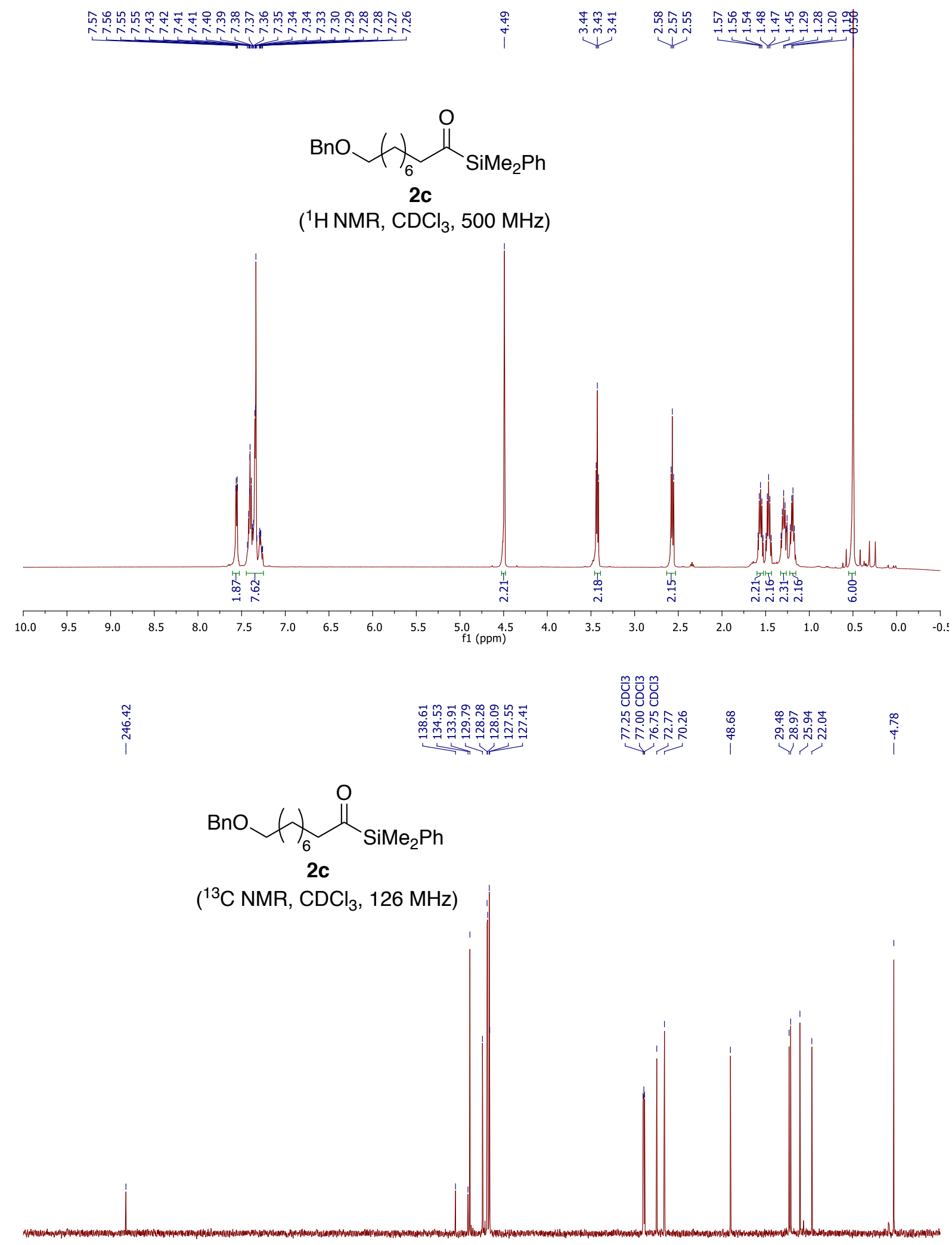

$\begin{array}{llllllllllllllllllllllllllllllllllllllll}270 & 260 & 250 & 240 & 230 & 220 & 210 & 200 & 190 & 180 & 170 & 160 & 150 & 140 & 130 & 120 & 110 & 100 & 90 & 80 & 70 & 60 & 50 & 40 & 30 & 20 & 10 & 0 & -10 & -20\end{array}$ 


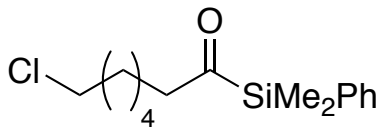

2d

( $\left.{ }^{1} \mathrm{HNMR}, \mathrm{CDCl}_{3}, 500 \mathrm{MHz}\right)$

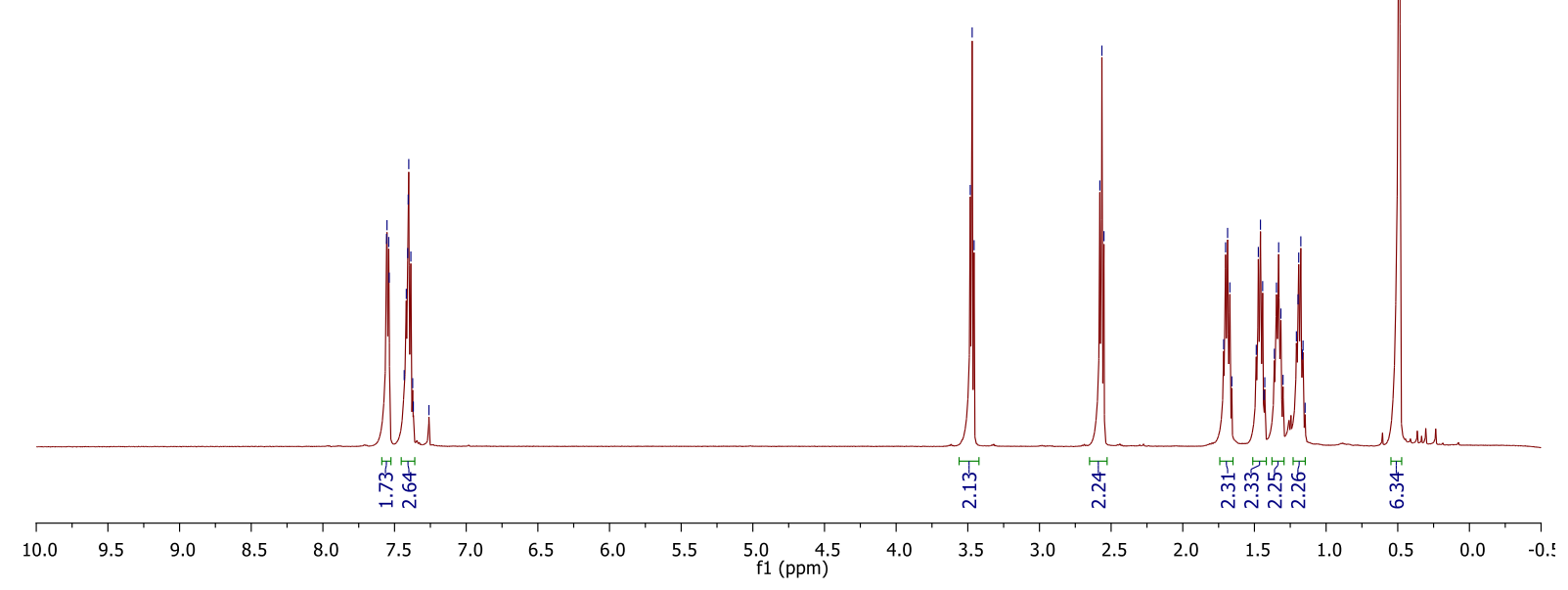<smiles>CC(C)(C)CC(=O)Sc1ccccc1</smiles>

2d

$\left({ }^{13} \mathrm{C} \mathrm{NMR}, \mathrm{CDCl}_{3}, 126 \mathrm{MHz}\right)$

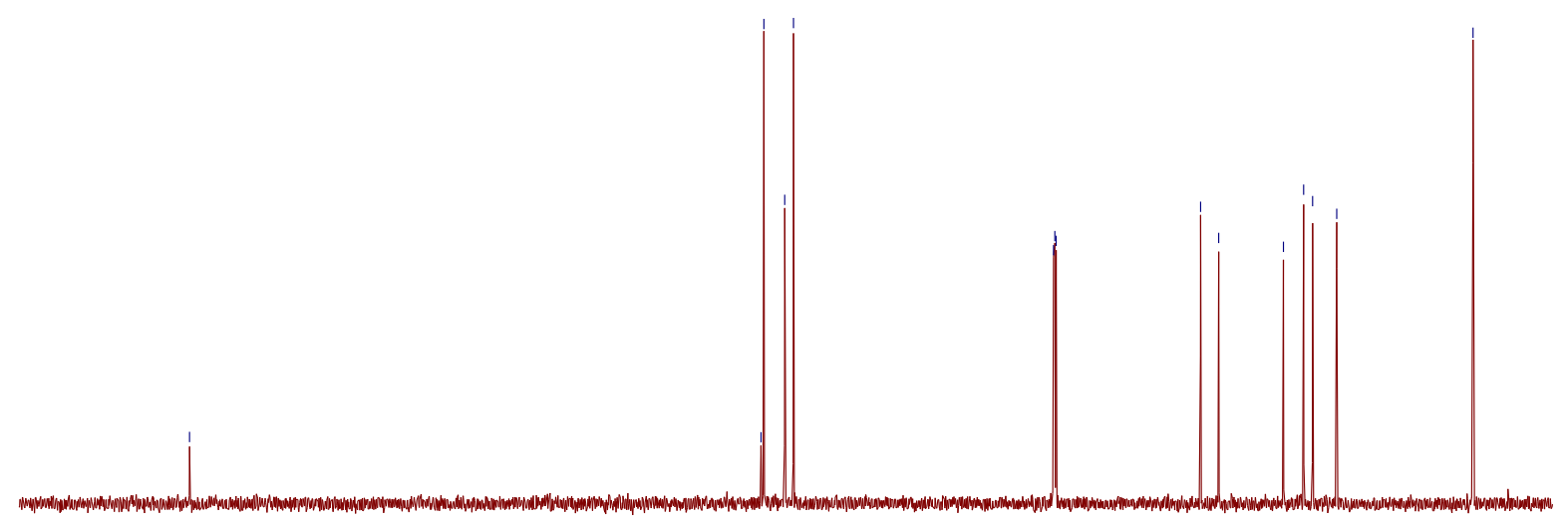

$\begin{array}{llllllllllllllllllllllllllllllllllllllllll}270 & 260 & 250 & 240 & 230 & 220 & 210 & 200 & 190 & 180 & 170 & 160 & 150 & 140 & 130 & 120 & 110 & 100 & 90 & 80 & 70 & 60 & 50 & 40 & 30 & 20 & 10 & 0 & -10 & -20\end{array}$ 

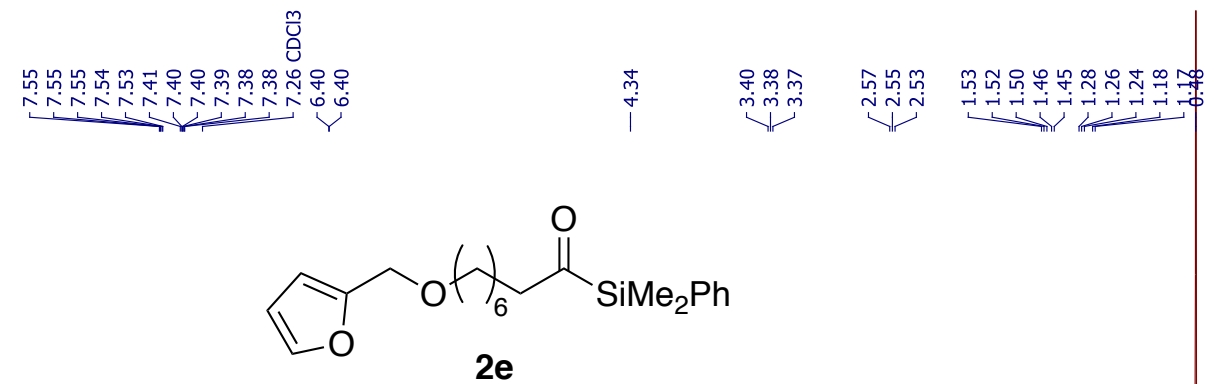

( $\left.{ }^{1} \mathrm{H} \mathrm{NMR}, \mathrm{CDCl}_{3}, 400 \mathrm{MHz}\right)$

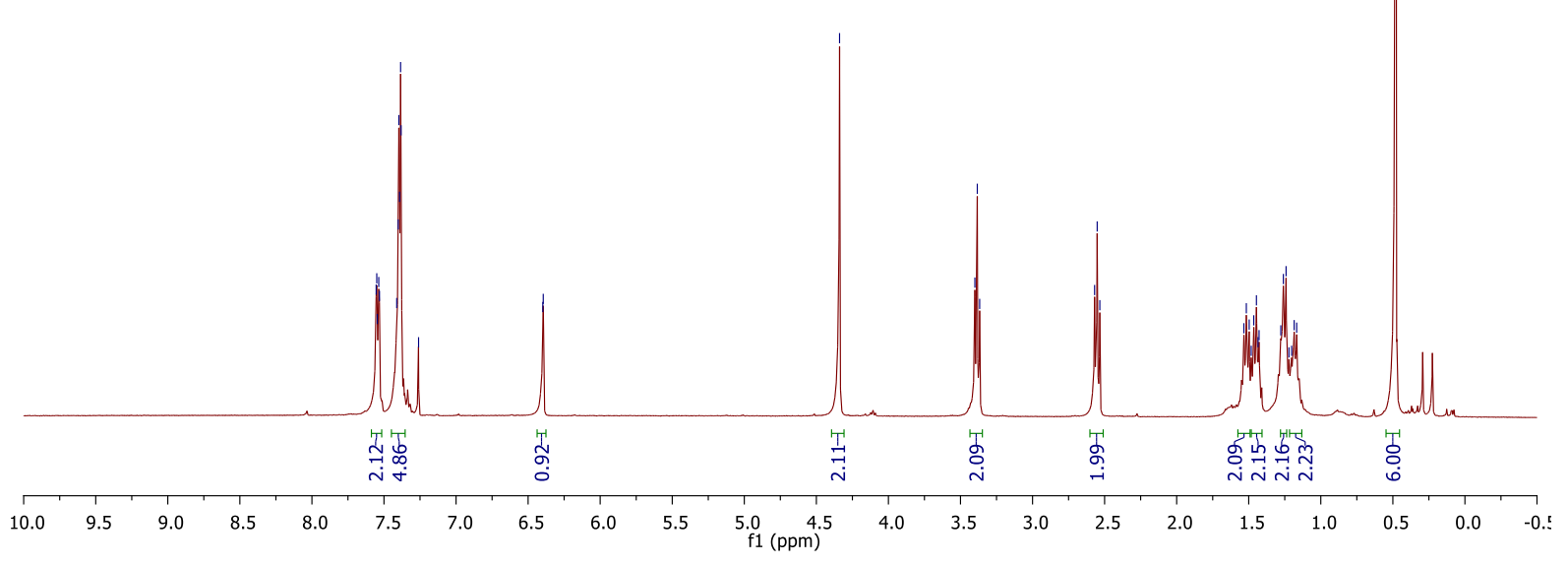

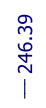

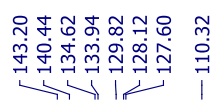

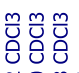
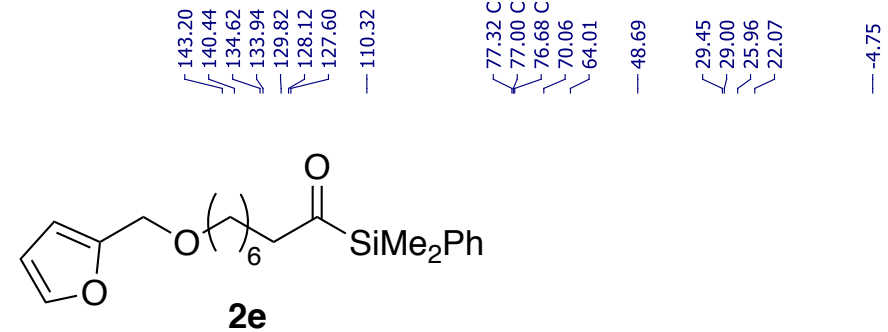

$\left({ }^{13} \mathrm{C} \mathrm{NMR}, \mathrm{CDCl}_{3}, 101 \mathrm{MHz}\right)$

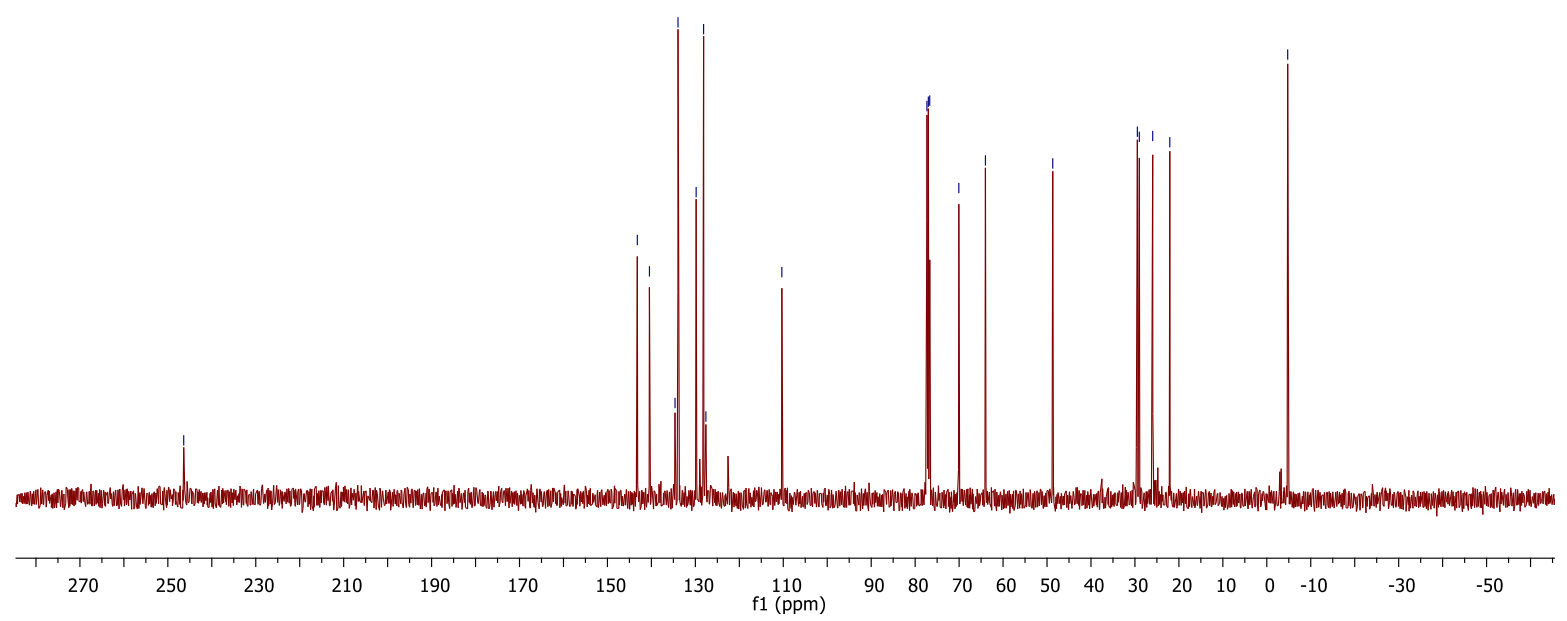




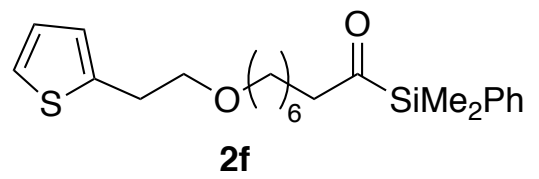

( ${ }^{1} \mathrm{HNMR}, \mathrm{CDCl}_{3}, 500 \mathrm{MHz}$ )

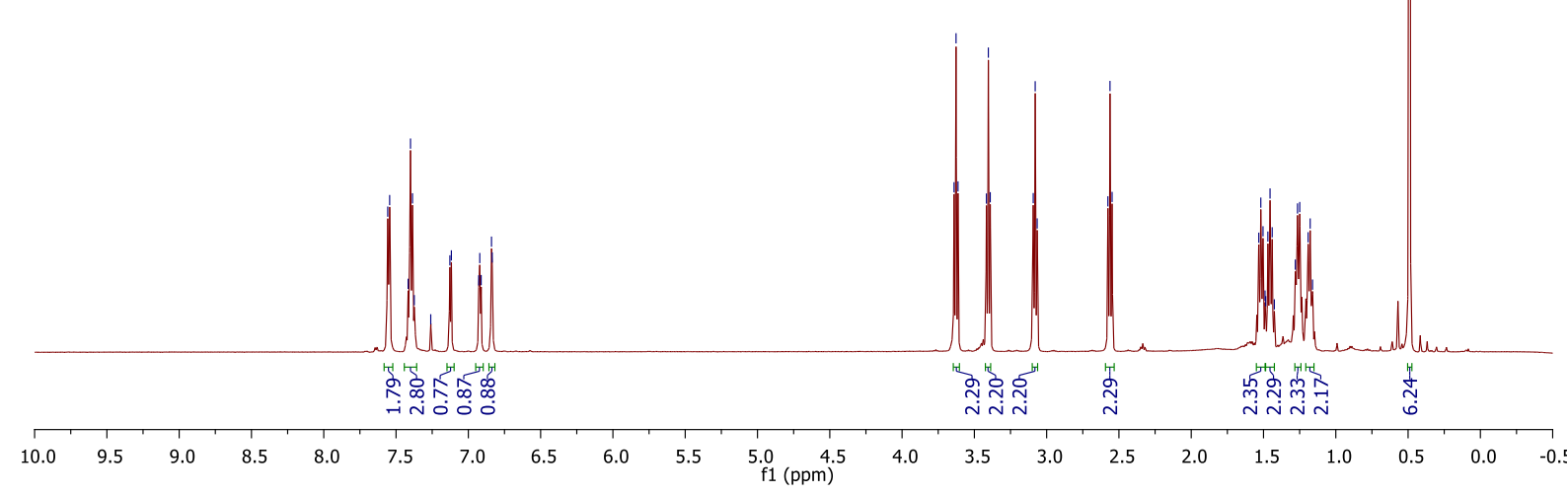

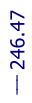
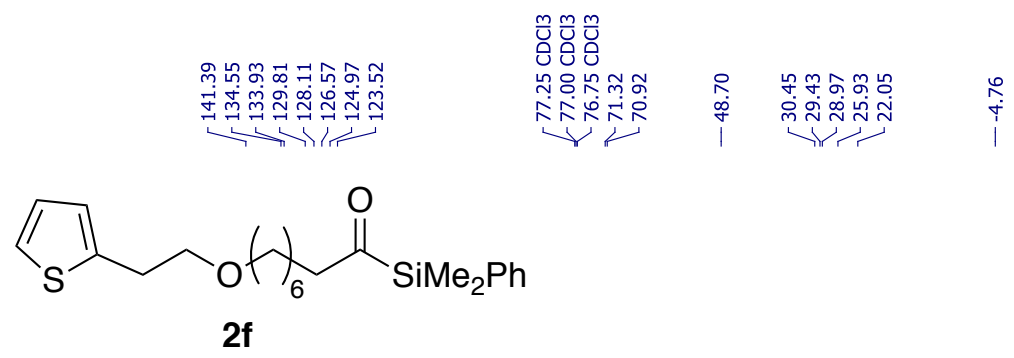

$\left({ }^{1} \mathrm{HNMR}, \mathrm{CDCl}_{3}, 126 \mathrm{MHz}\right)$

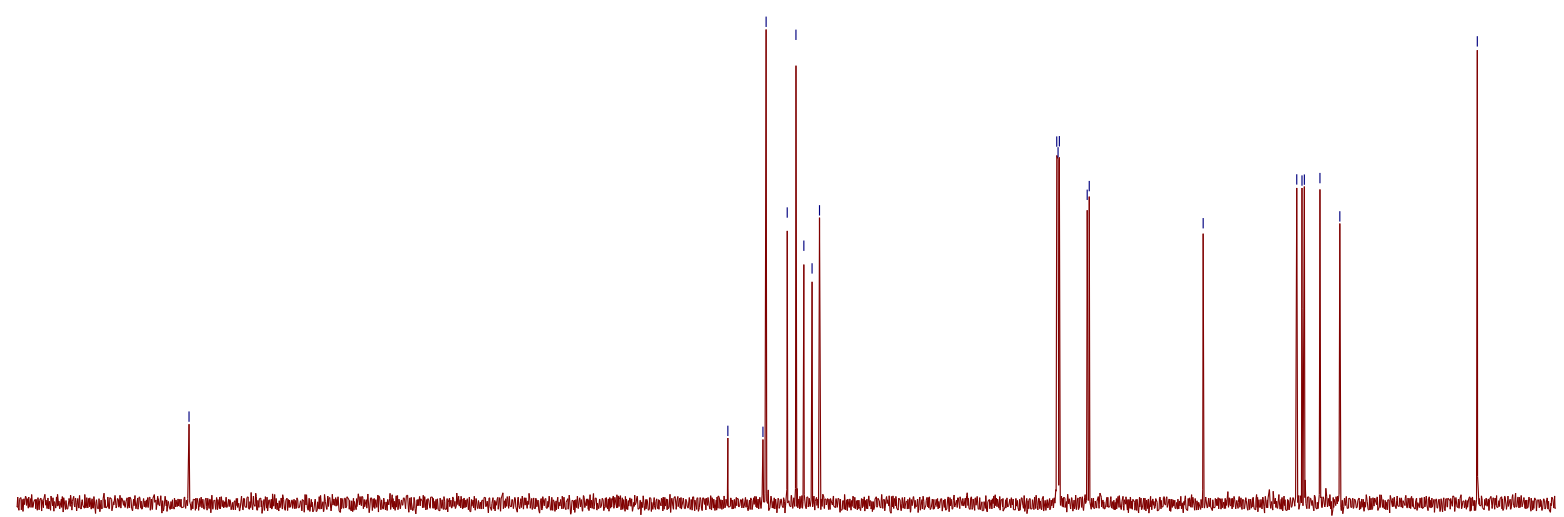

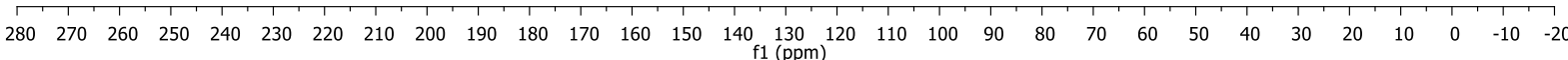



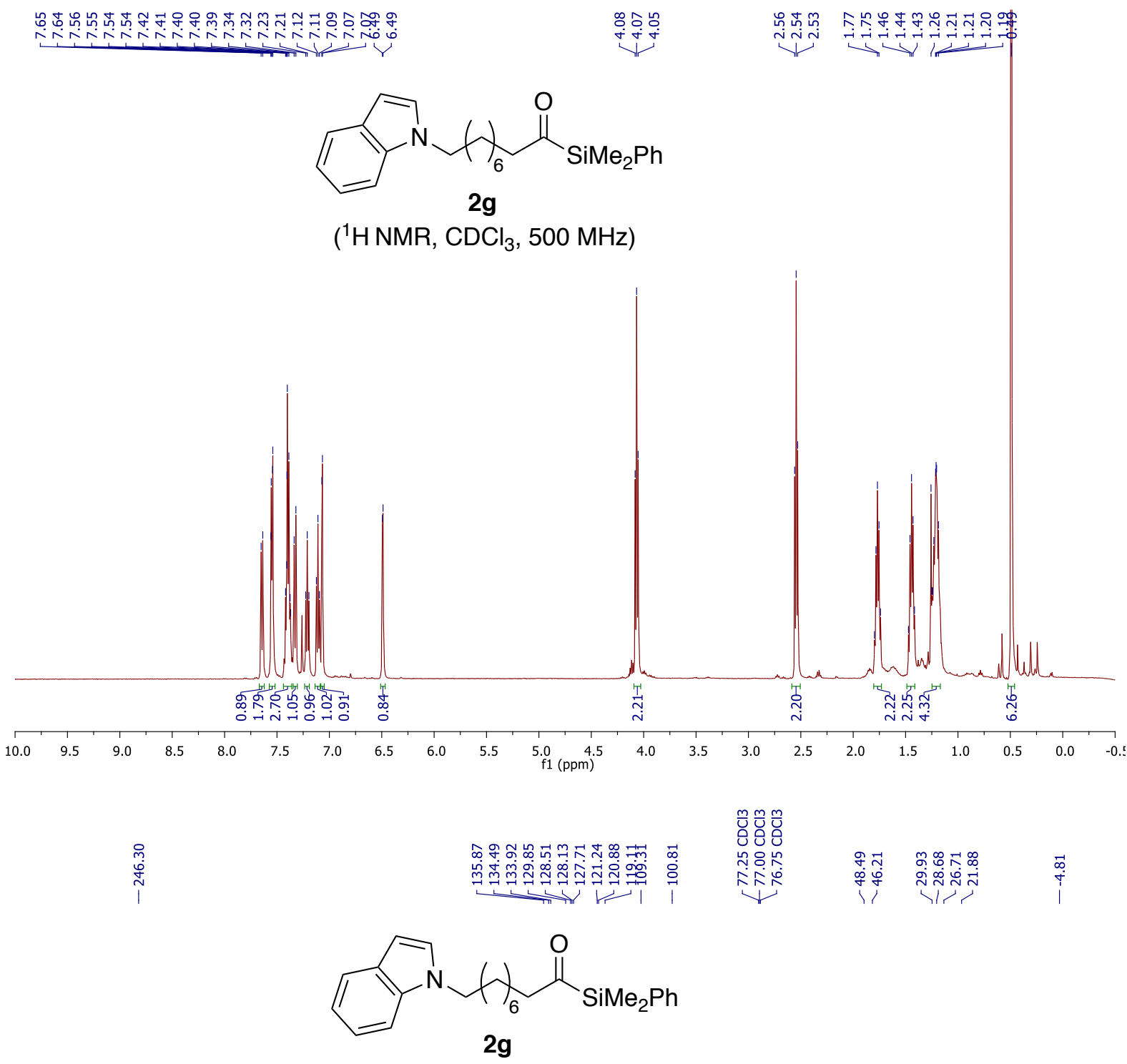

$\left({ }^{13} \mathrm{C} \mathrm{NMR}, \mathrm{CDCl}_{3}, 126 \mathrm{MHz}\right)$

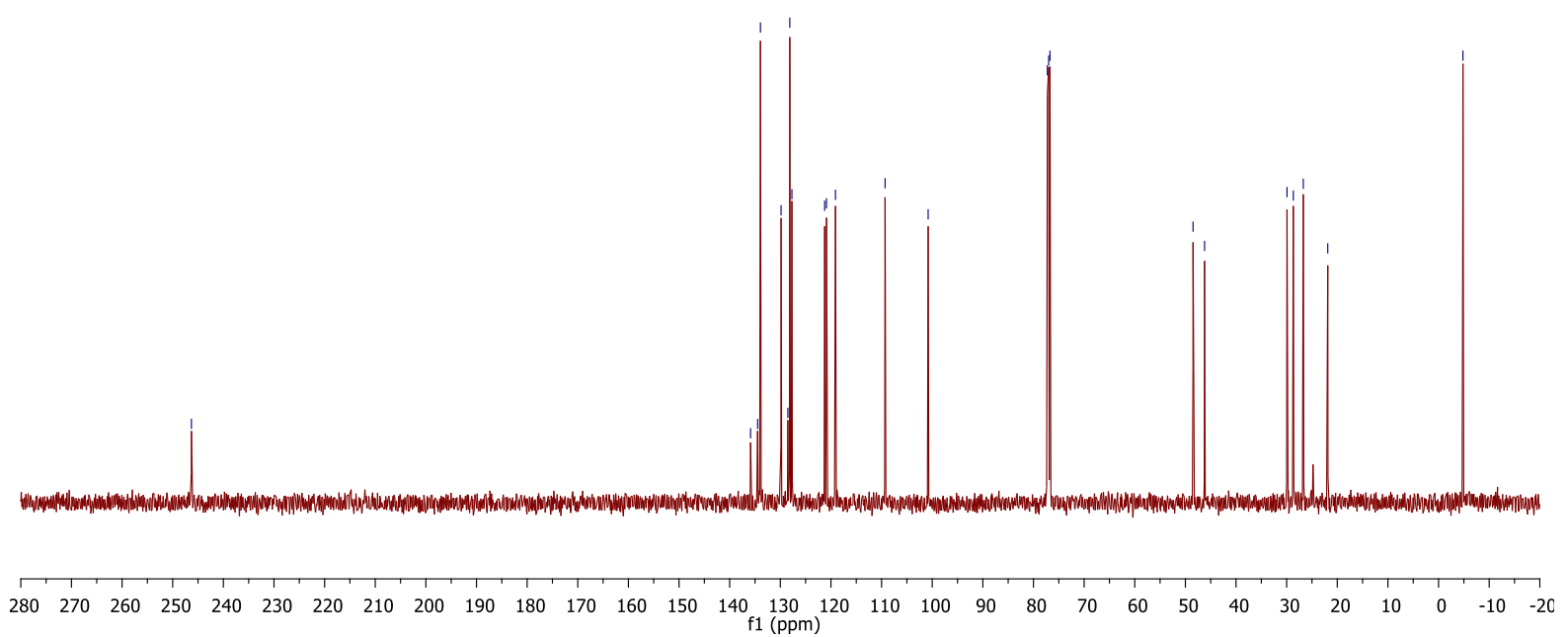




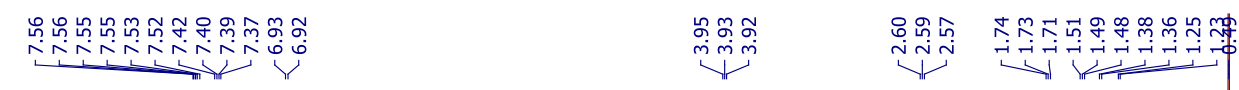

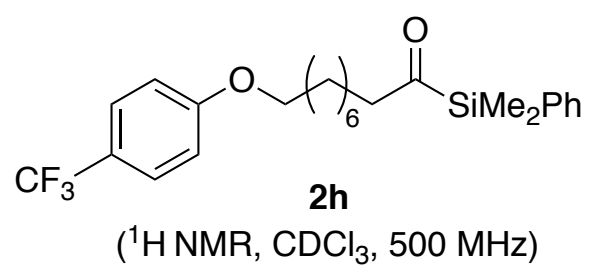
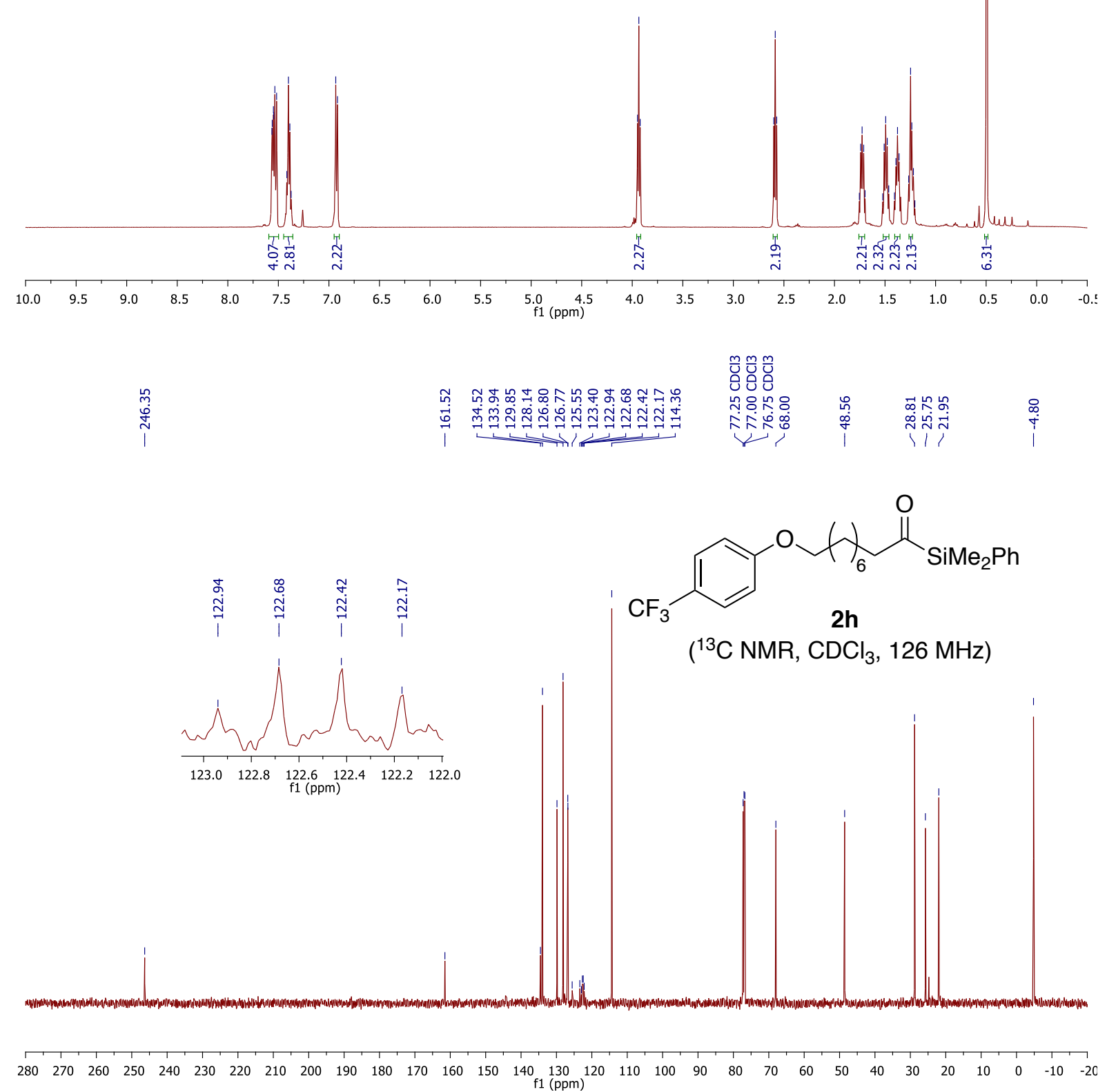

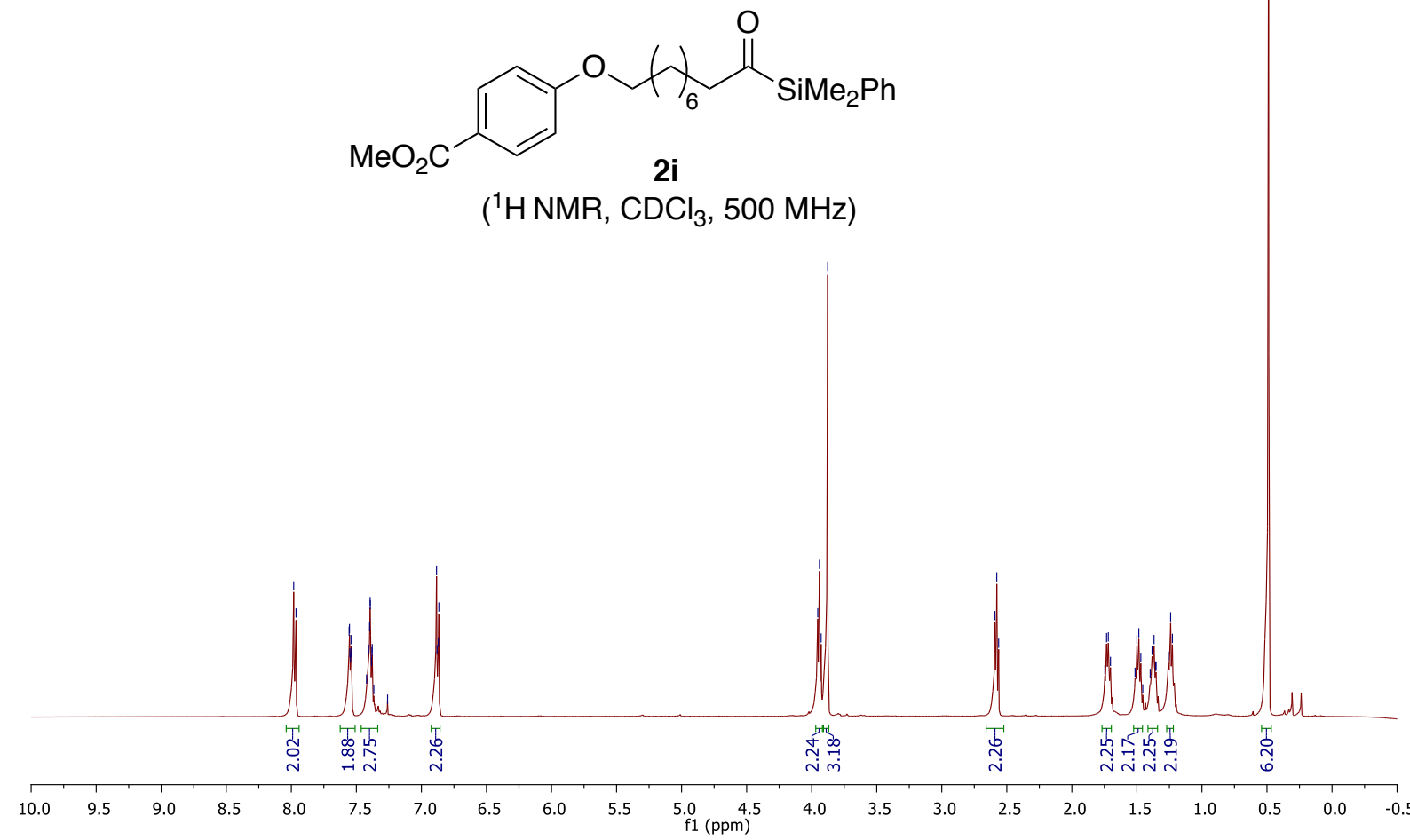

$\stackrel{\vec{m}}{\stackrel{i}{\infty}}$

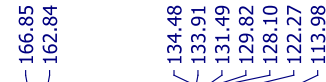

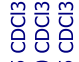

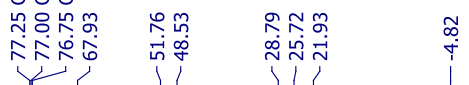<smiles>CC(=O)c1ccc(OCC(C)CCC(=O)SCc2ccccc2)cc1</smiles>

$\left({ }^{13} \mathrm{C} \mathrm{NMR}, \mathrm{CDCl}_{3}, 126 \mathrm{MHz}\right)$

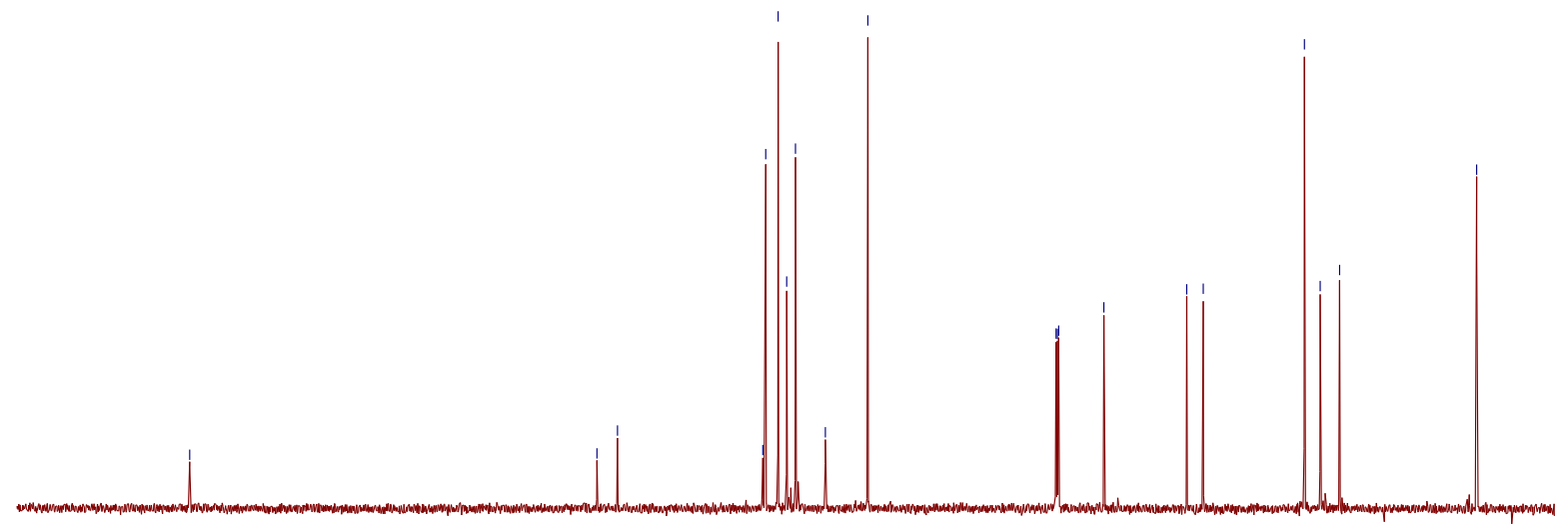

$\begin{array}{llllllllllllllllllllllllllllllllllllll}280 & 270 & 260 & 250 & 240 & 230 & 220 & 210 & 200 & 190 & 180 & 170 & 160 & 150 & 140 & 130 & 120 & 110 & 100 & 90 & 80 & 70 & 60 & 50 & 40 & 30 & 20 & 10 & 0 & -10 & -20\end{array}$ 

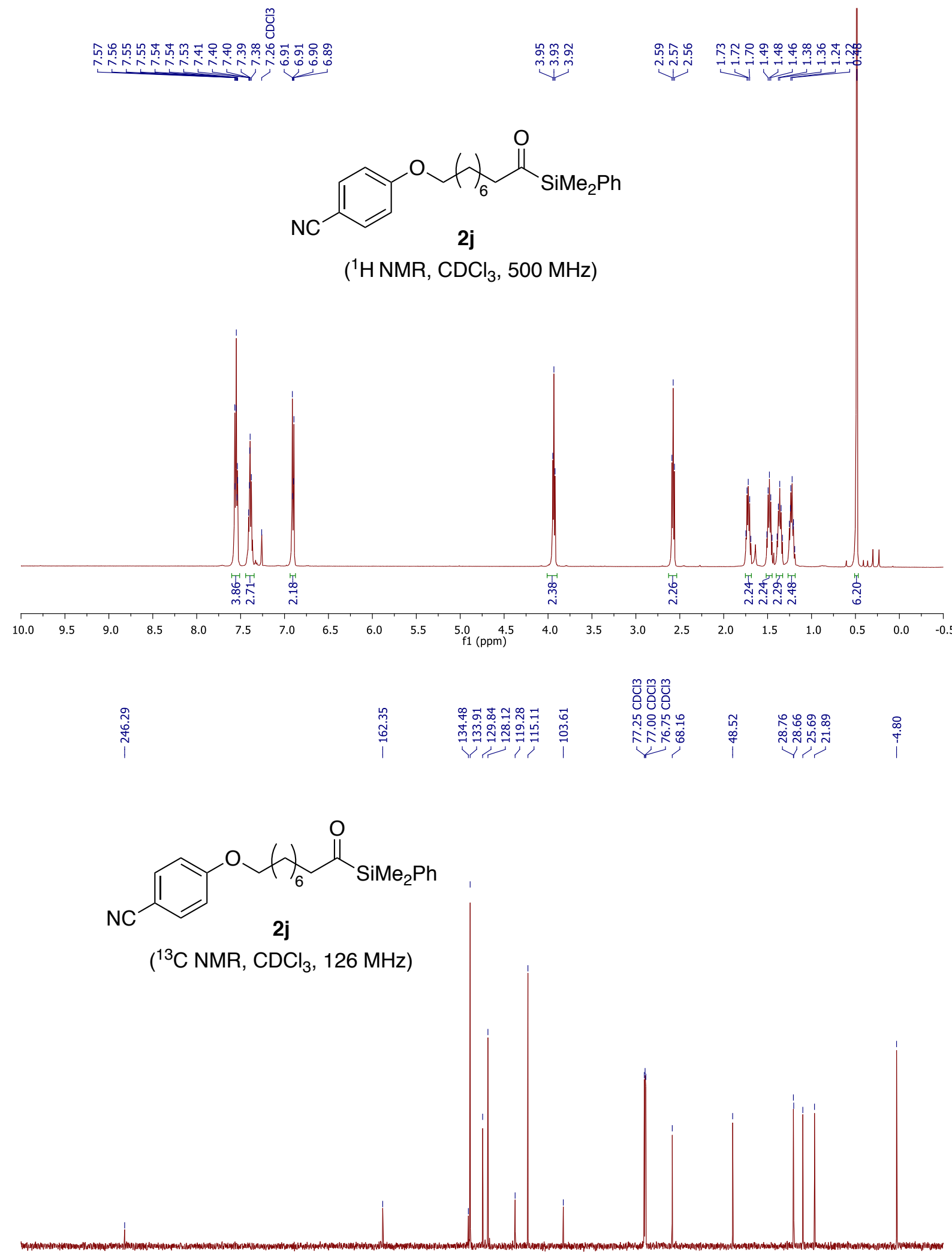

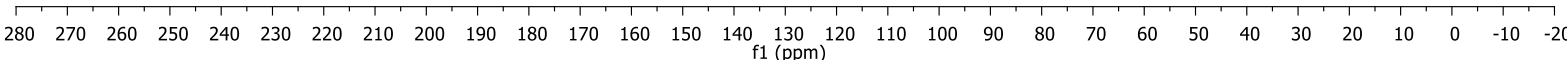



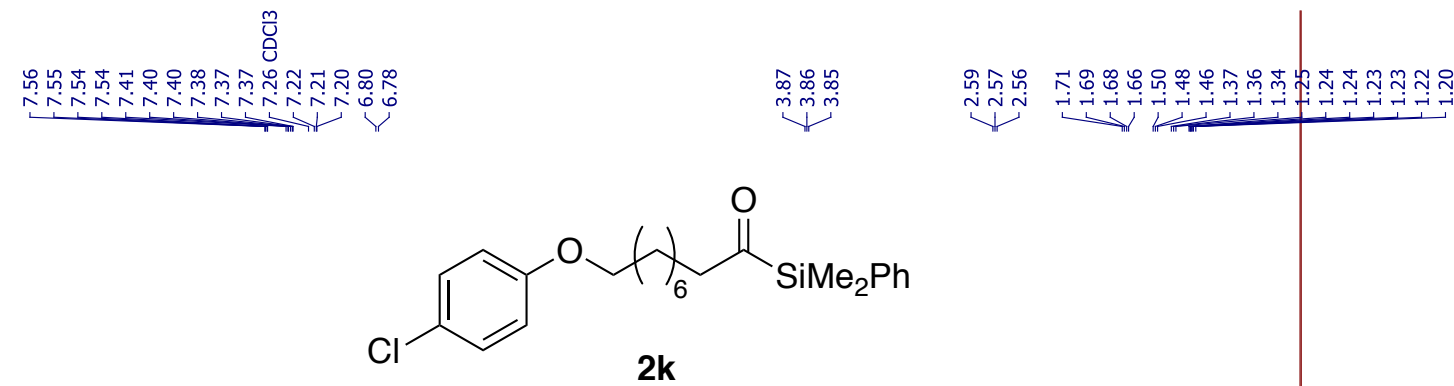

$\left({ }^{1} \mathrm{HNMR}, \mathrm{CDCl}_{3}, 500 \mathrm{MHz}\right)$
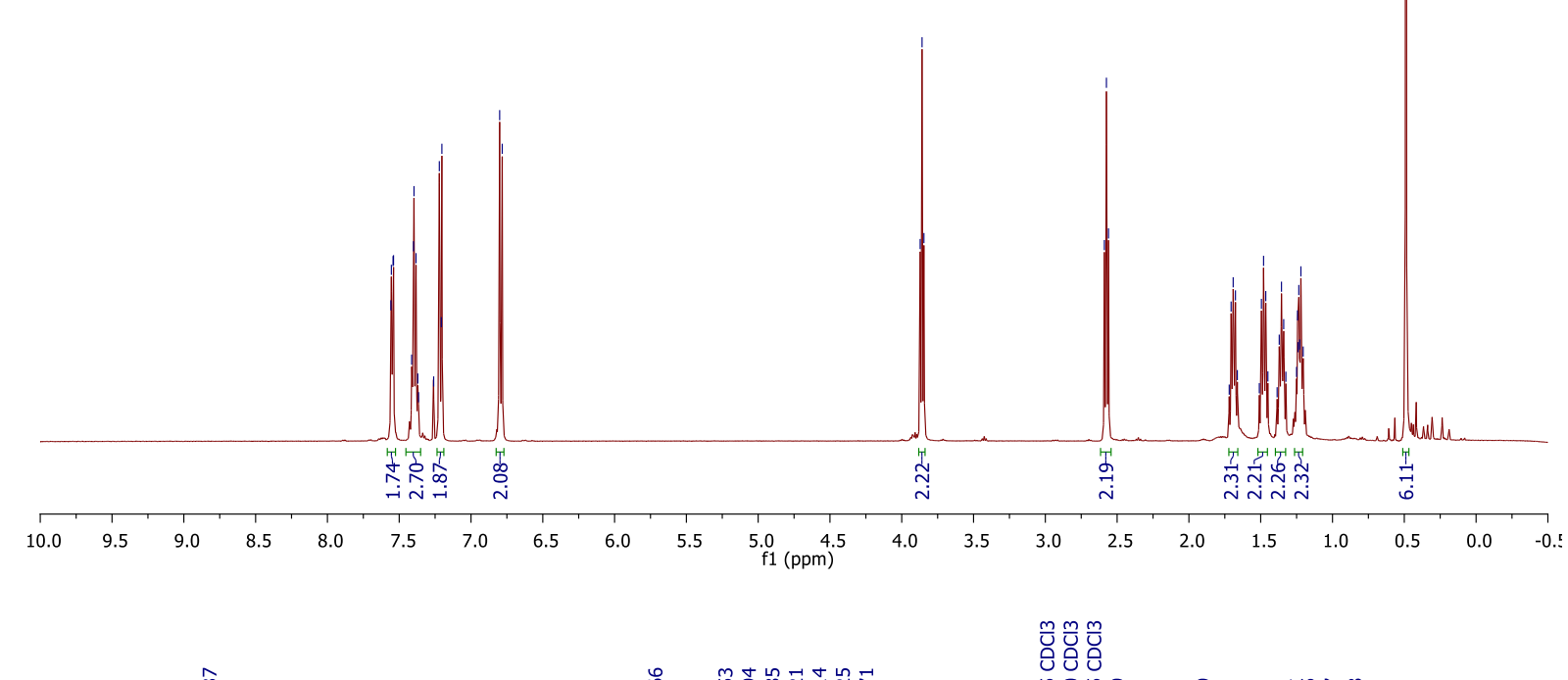

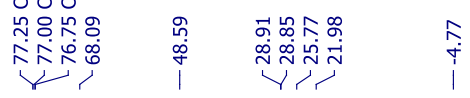

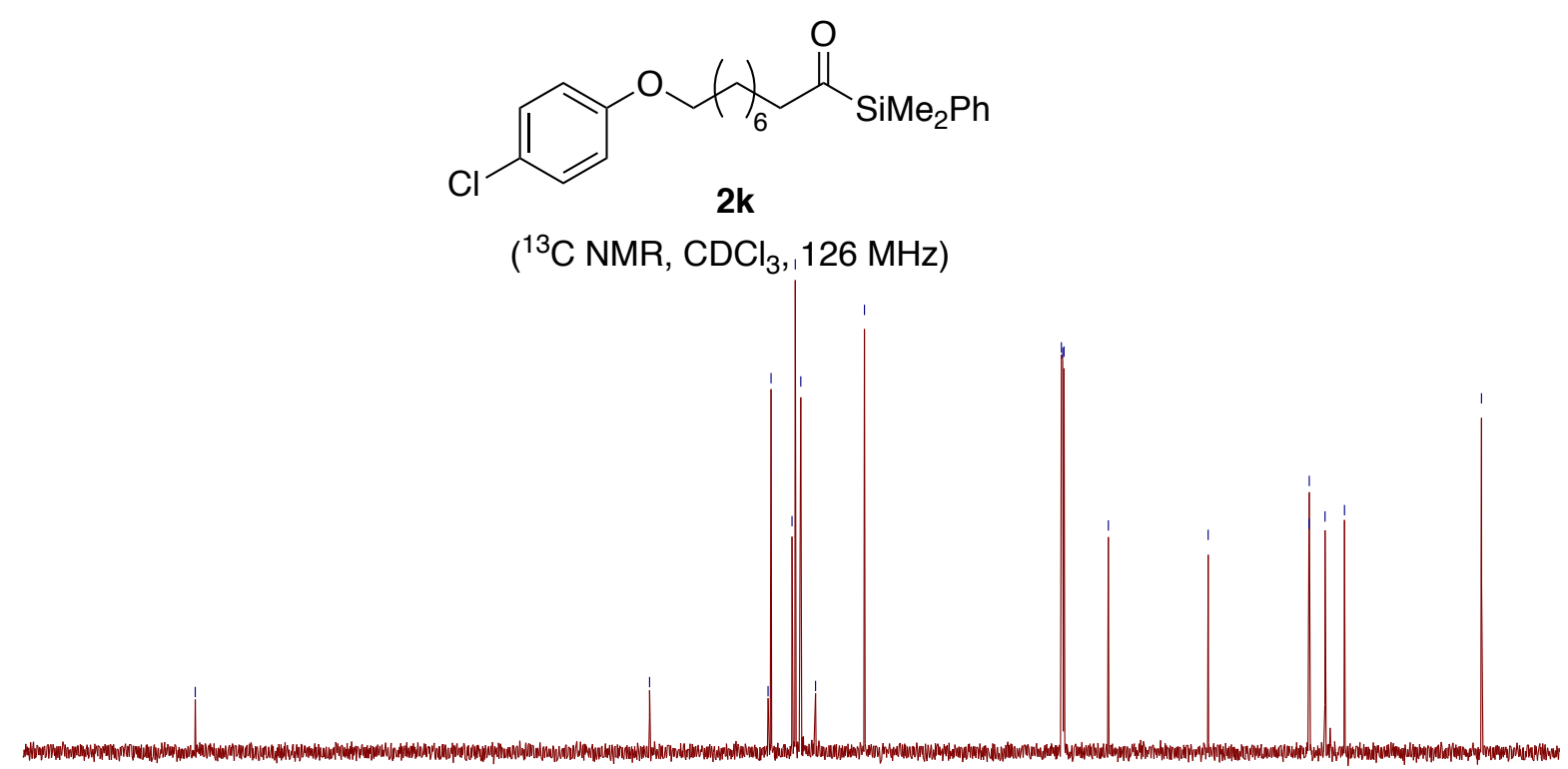

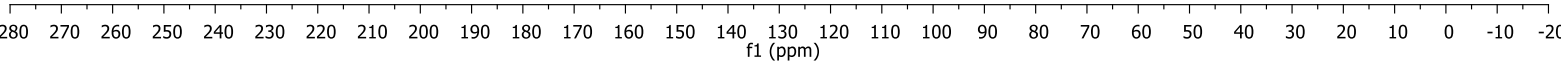



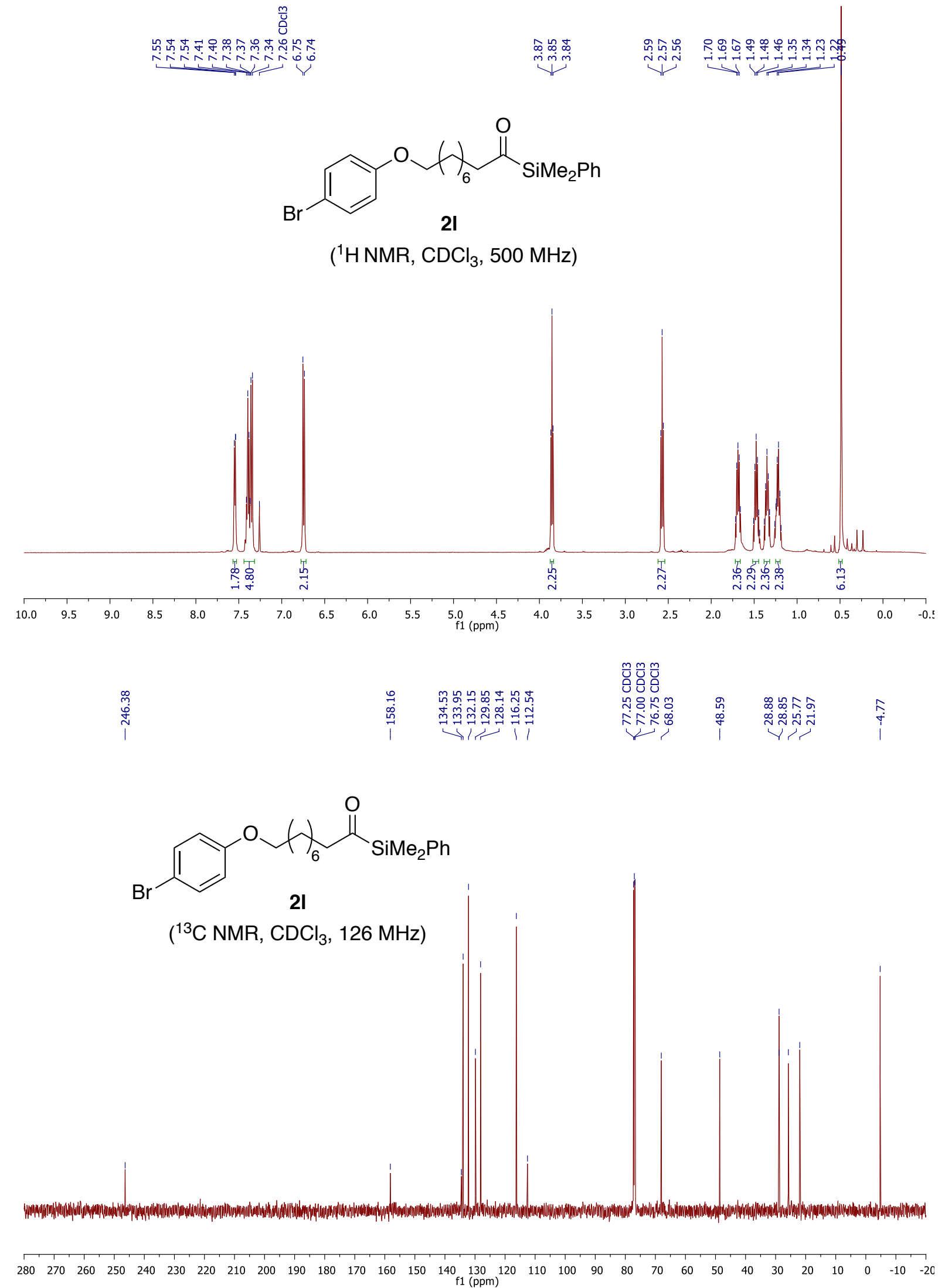


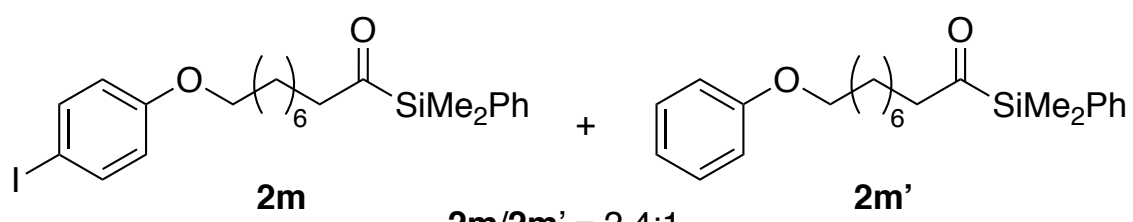

$2 m$

$2 \mathrm{~m} / \mathbf{2} \mathrm{m}^{\prime}=2.4: 1$

$2 m^{\prime}$

( ${ }^{1} \mathrm{H} \mathrm{NMR}, \mathrm{CDCl}_{3}, 500 \mathrm{MHz}$ )
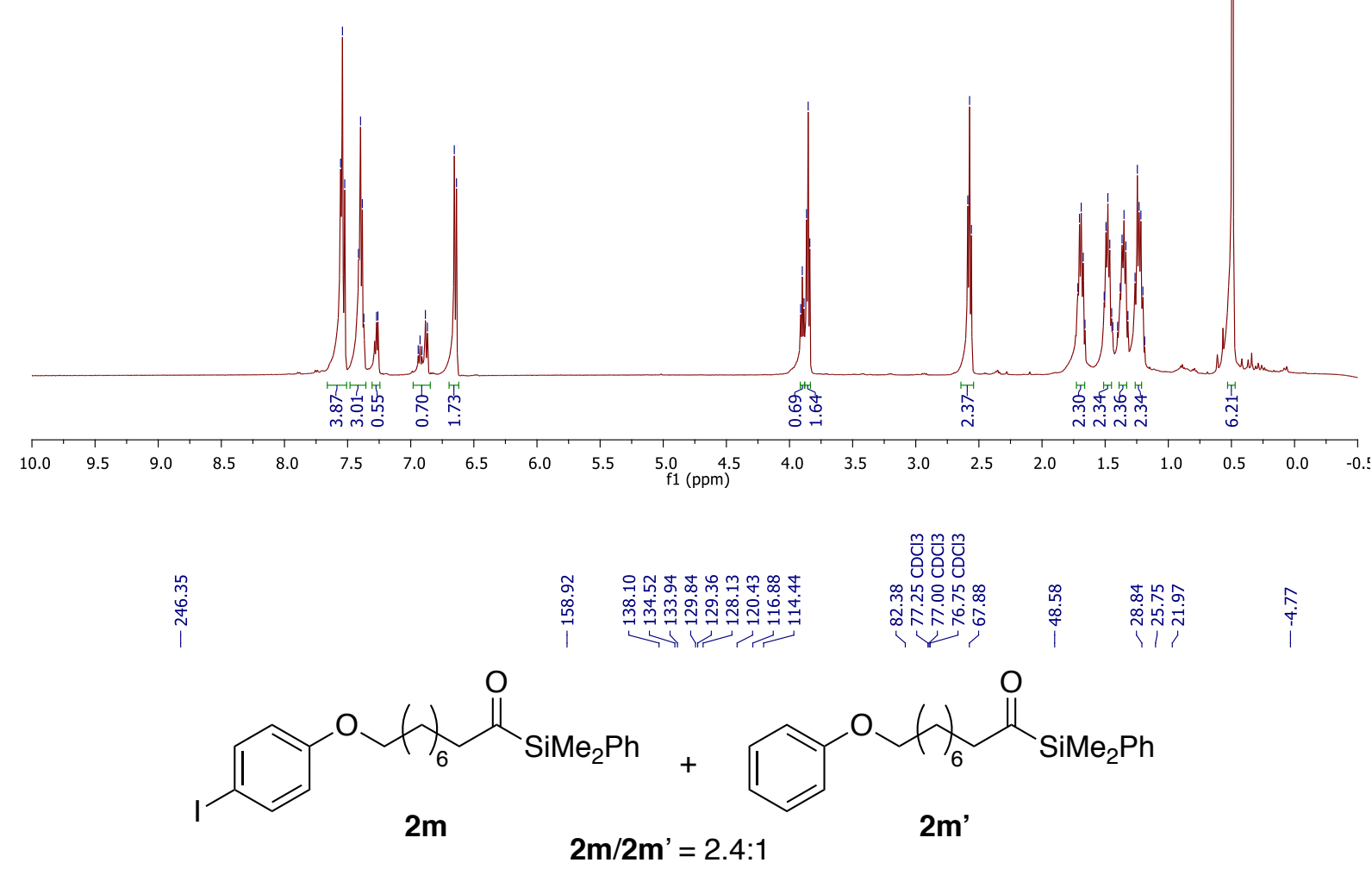

$\left({ }^{13} \mathrm{C} \mathrm{NMR}, \mathrm{CDCl}_{3}, 126 \mathrm{MHz}\right)$

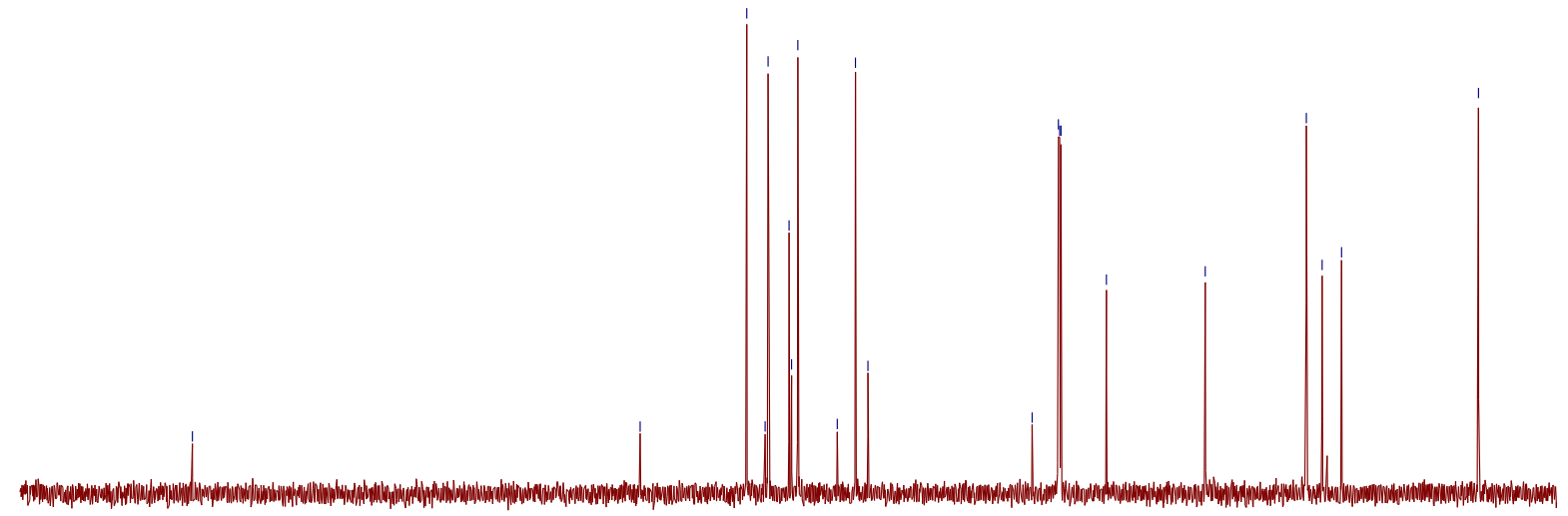

$\begin{array}{llllllllllllllllllllllllllllllllllll}280 & 270 & 260 & 250 & 240 & 230 & 220 & 210 & 200 & 190 & 180 & 170 & 160 & 150 & 140 & 130 & 120 & 110 & 100 & 90 & 80 & 70 & 60 & 50 & 40 & 30 & 20 & 10 & 0 & -10 & -2\end{array}$ 


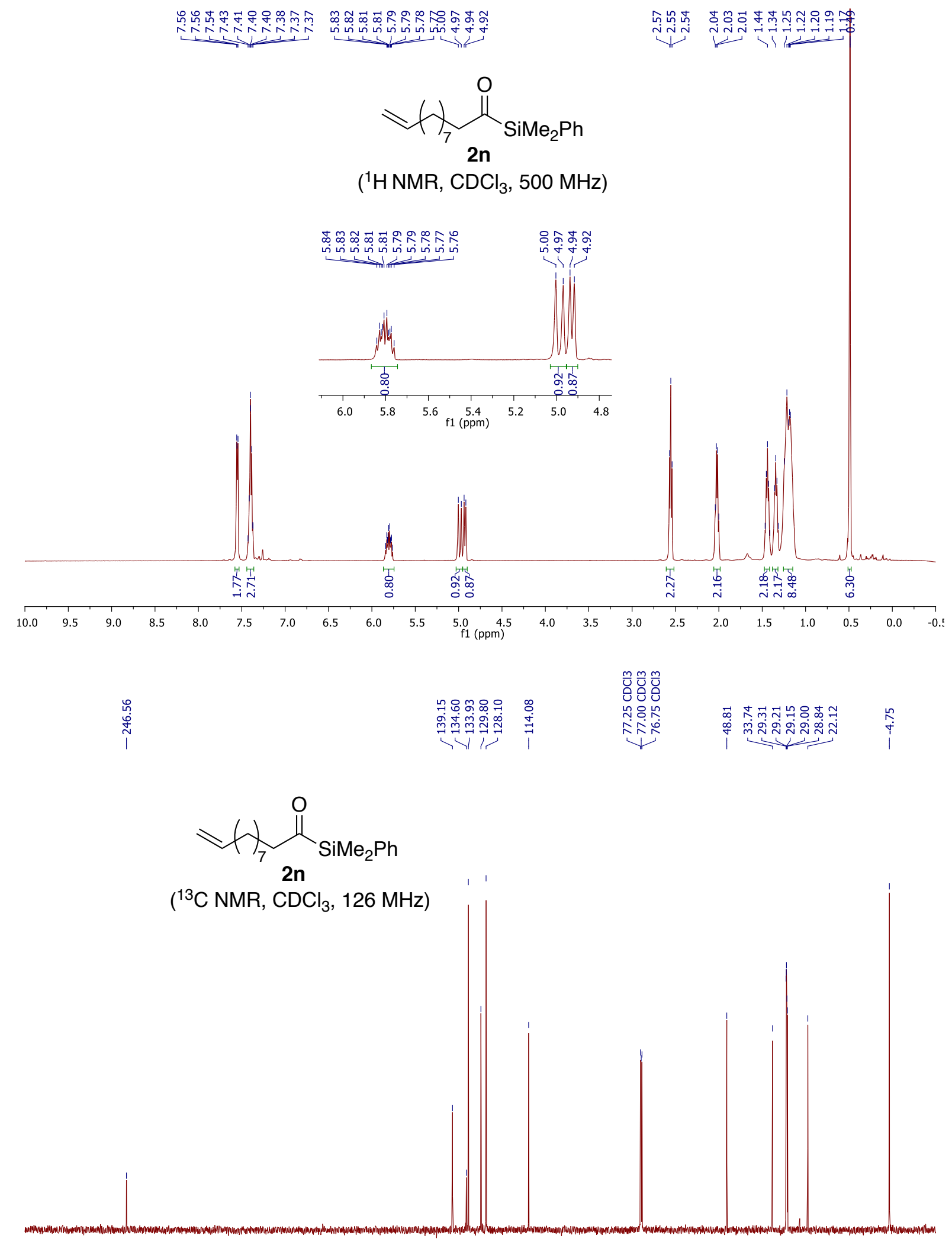

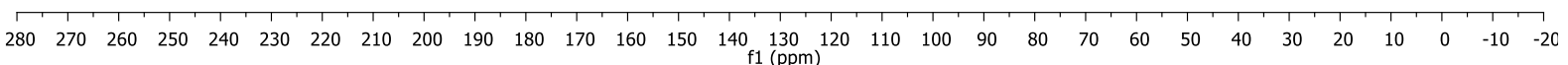




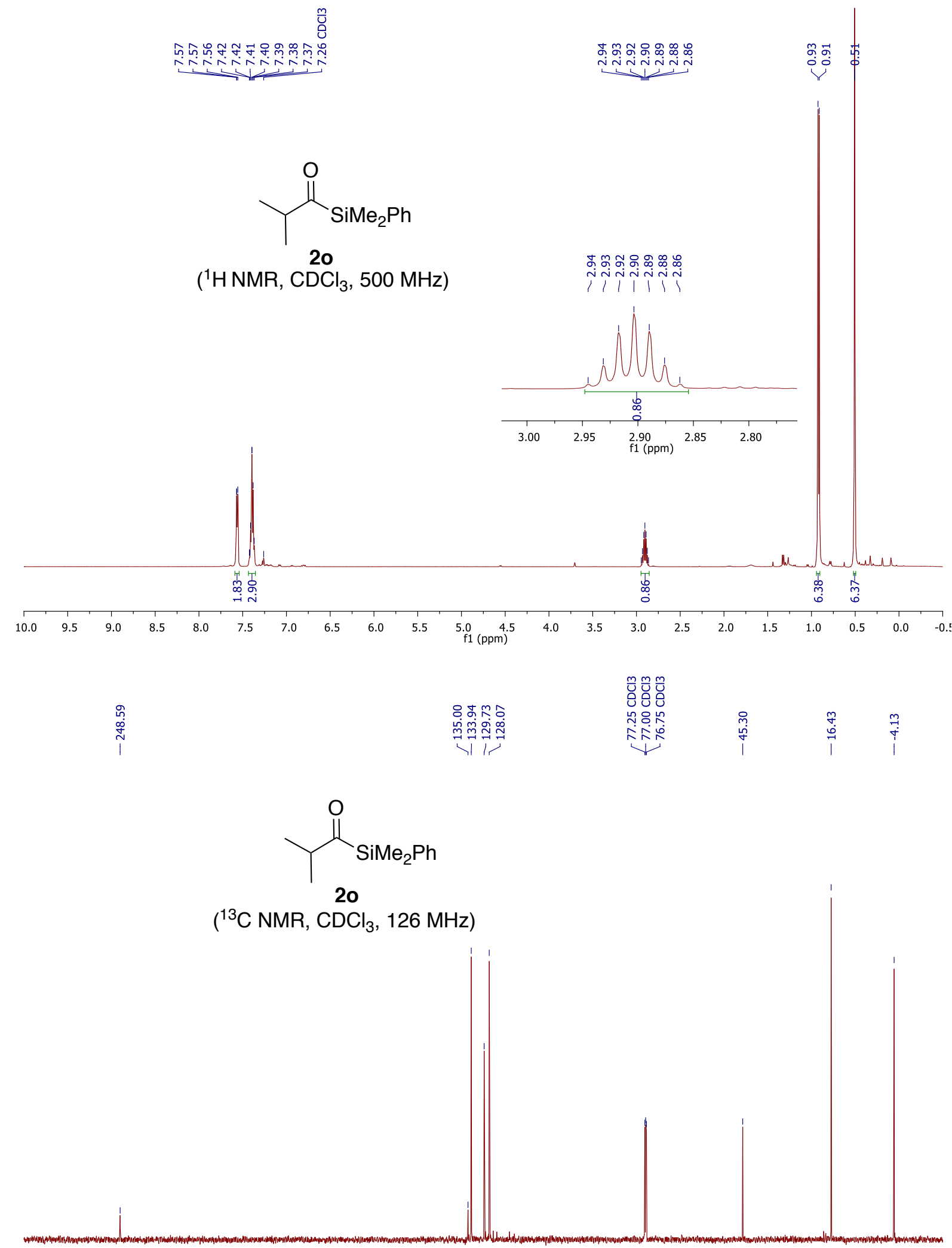

\begin{tabular}{rlllllllllllllllllllllllllllllllllllllllllllllll}
\hline 280 & 270 & 260 & 250 & 240 & 230 & 220 & 210 & 200 & 190 & 180 & 170 & 160 & 150 & 140 & 130 & 120 & 110 & 100 & 90 & 80 & 70 & 60 & 50 & 40 & 30 & 20 & 10 & 0 & -10 & -20
\end{tabular} 


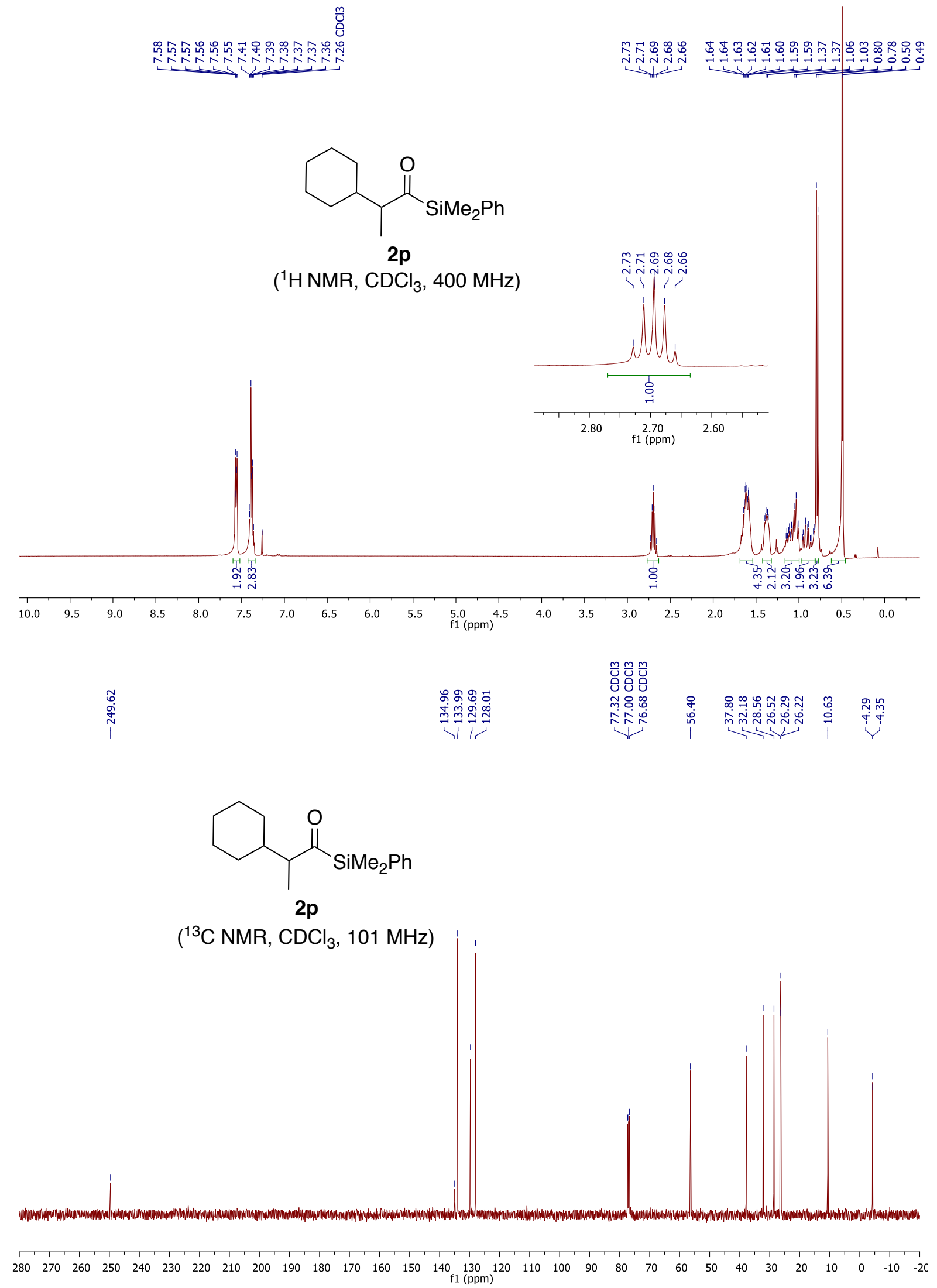




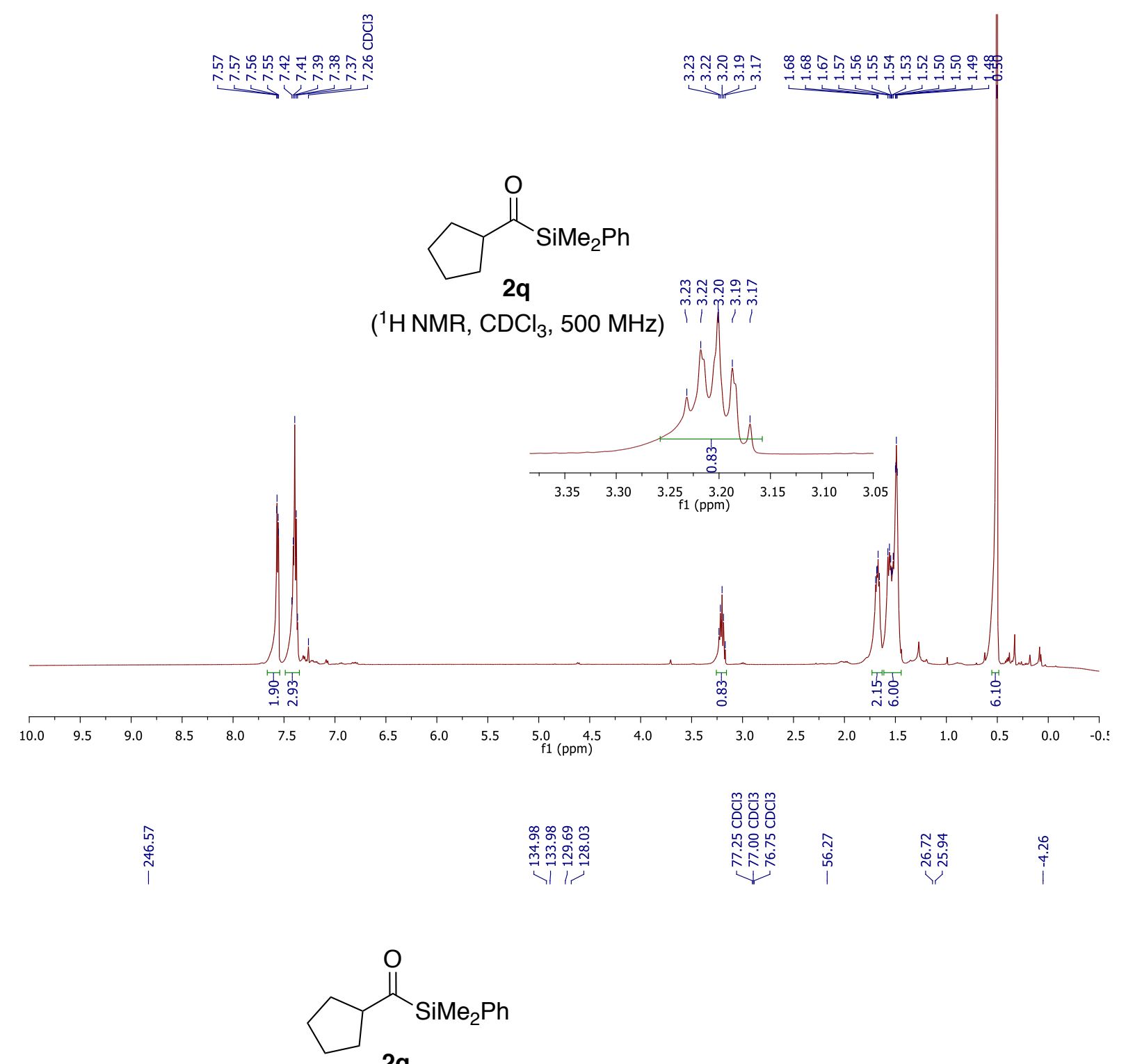

$2 q$

$\left({ }^{13} \mathrm{C} \mathrm{NMR}, \mathrm{CDCl}_{3}, 126 \mathrm{MHz}\right)$

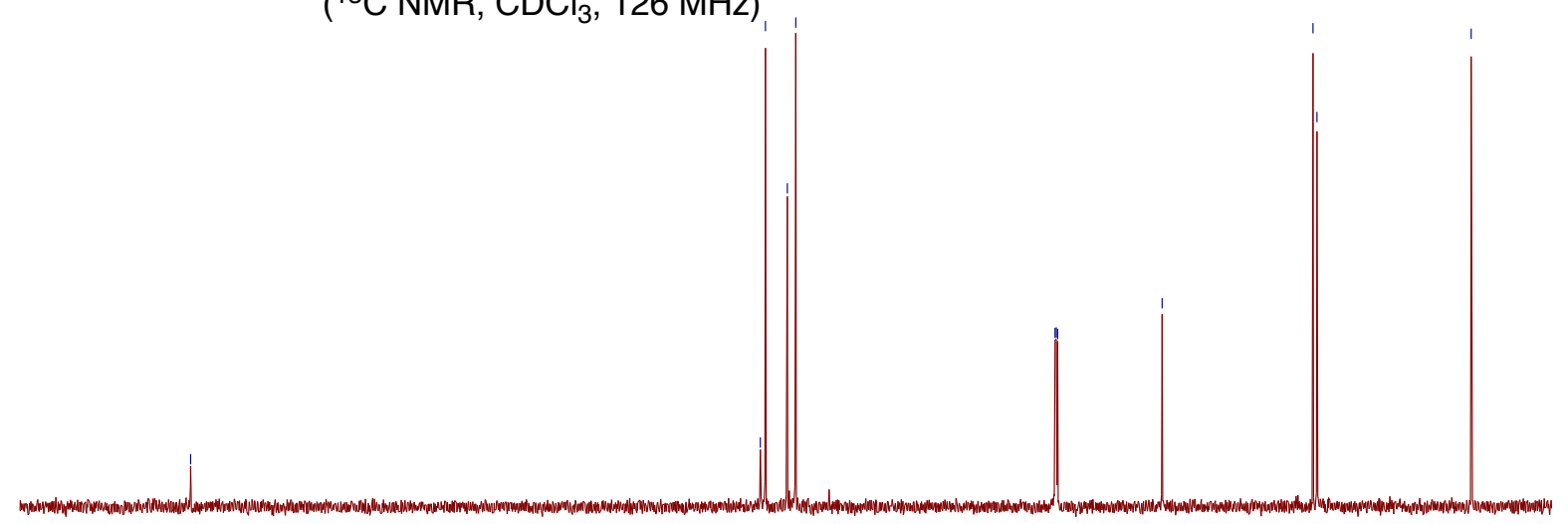

\begin{tabular}{rllllllllllllllllllllllllllllllllllllllllllllll}
\hline 280 & 270 & 260 & 250 & 240 & 230 & 220 & 210 & 200 & 190 & 180 & 170 & 160 & 150 & 140 & 130 & 120 & 110 & 100 & 90 & 80 & 70 & 60 & 50 & 40 & 30 & 20 & 10 & 0 & -10 & -20
\end{tabular} 


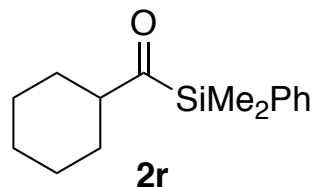

$\left({ }^{1} \mathrm{HNMR}, \mathrm{CDCl}_{3}, 500 \mathrm{MHz}\right)$
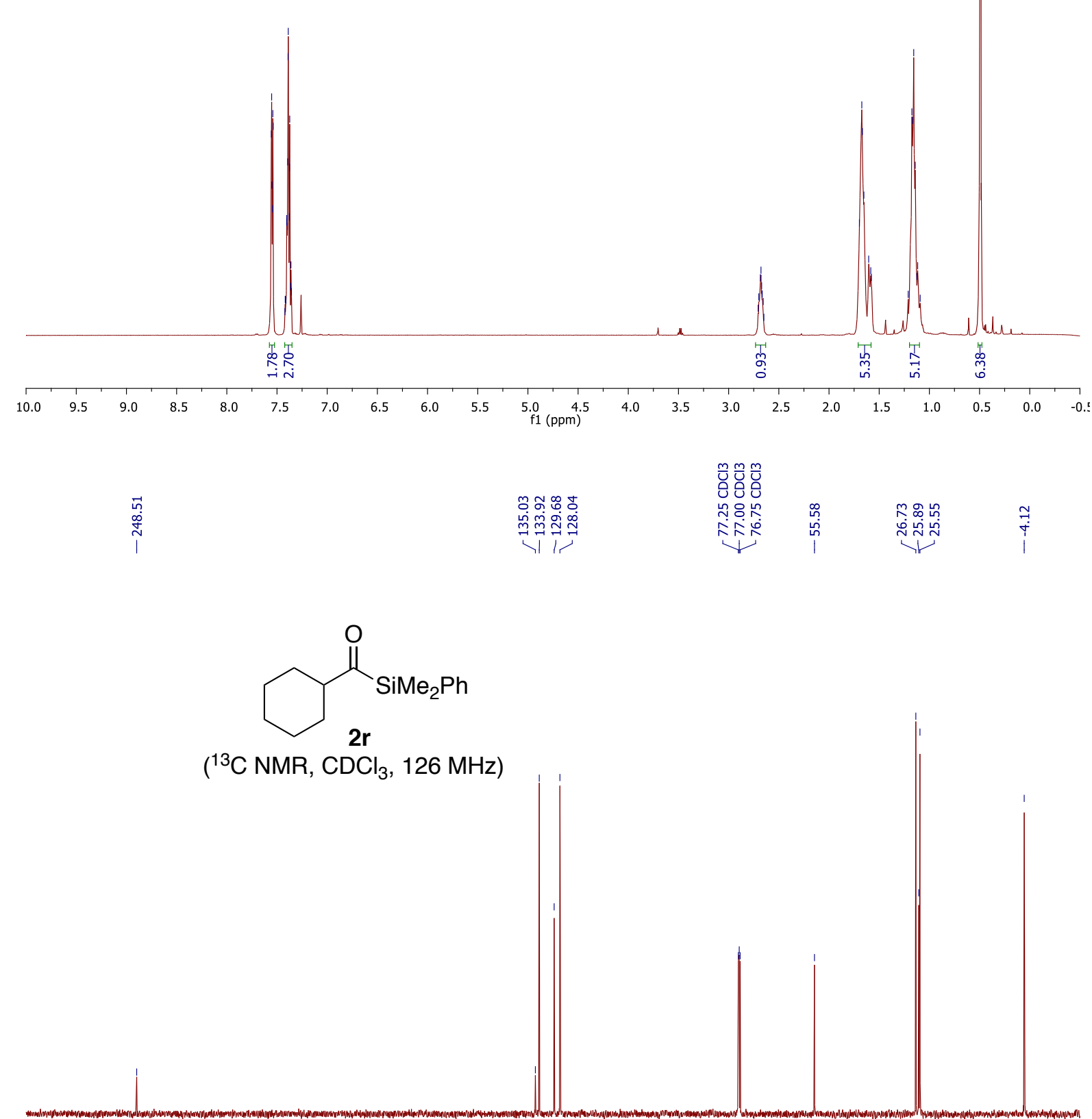

$\begin{array}{llllllllllllllllllllllllllllllllll}280 & 270 & 260 & 250 & 240 & 230 & 220 & 210 & 200 & 190 & 180 & 170 & 160 & 150 & 140 & 130 & 120 & 110 & 100 & 90 & 80 & 70 & 60 & 50 & 40 & 30 & 20 & 10 & 0 & -10 & -20\end{array}$ 
<smiles>O=C(C1CCOCC1)C1CCOCC1</smiles>

2s

( $\left.{ }^{1} \mathrm{HNMR}, \mathrm{CDCl}_{3}, 500 \mathrm{MHz}\right)$

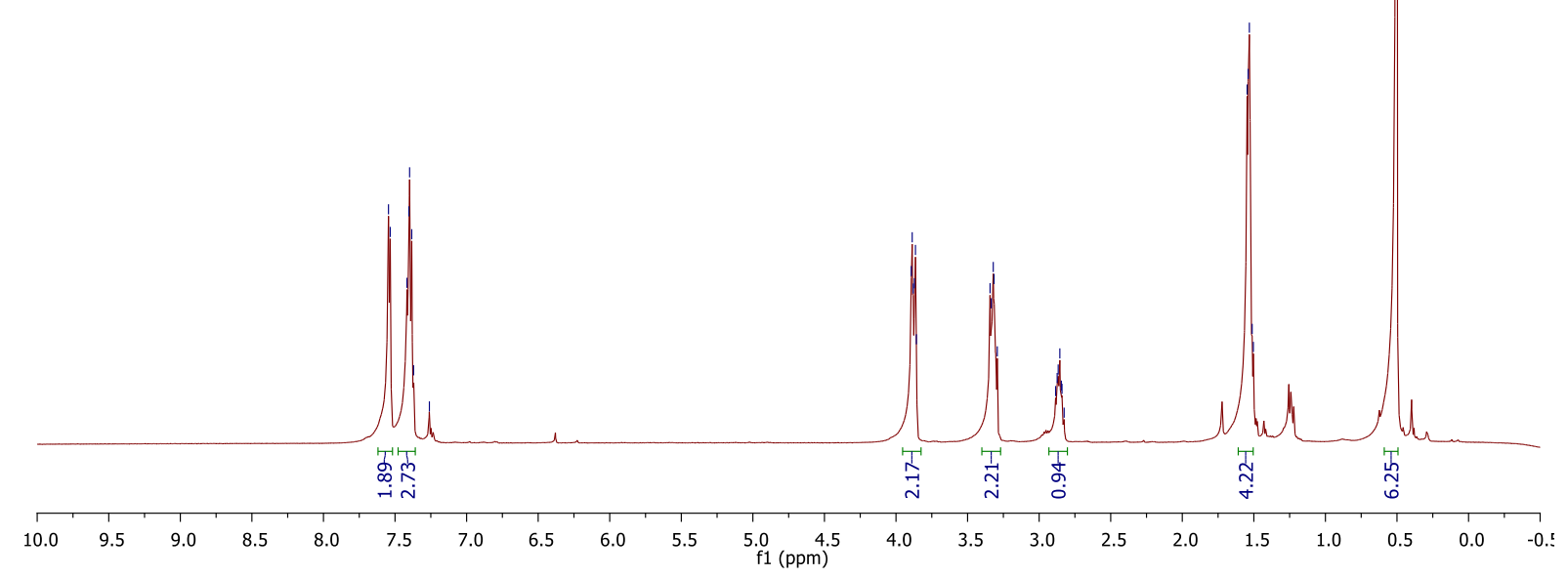<smiles>O=C(C1CCOCC1)C1CCOCC1</smiles>

2s

$\left({ }^{13} \mathrm{C} \mathrm{NMR}, \mathrm{CDCl}_{3}, 126 \mathrm{MHz}\right)$

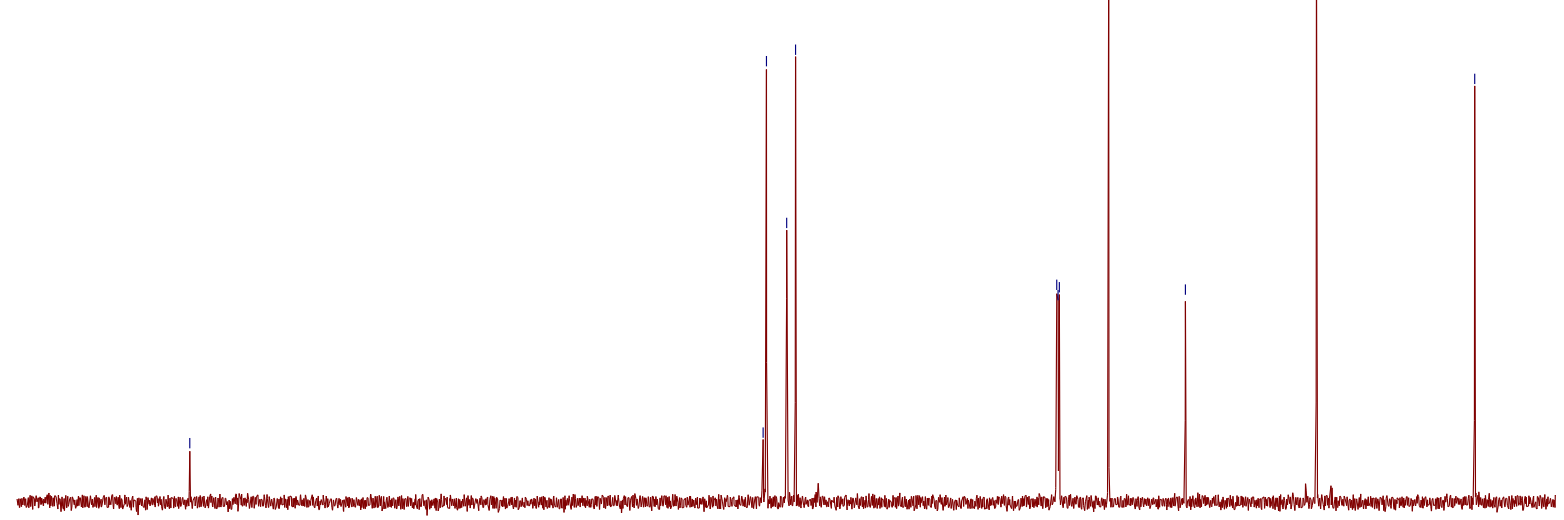

$\begin{array}{rlllllllllllllllllllllllllllllllllllllllllllllll}280 & 270 & 260 & 250 & 240 & 230 & 220 & 210 & 200 & 190 & 180 & 170 & 160 & 150 & 140 & 130 & 120 & 110 & 100 & 90 & 80 & 70 & 60 & 50 & 40 & 30 & 20 & 10 & 0 & -10 & -20\end{array}$ 

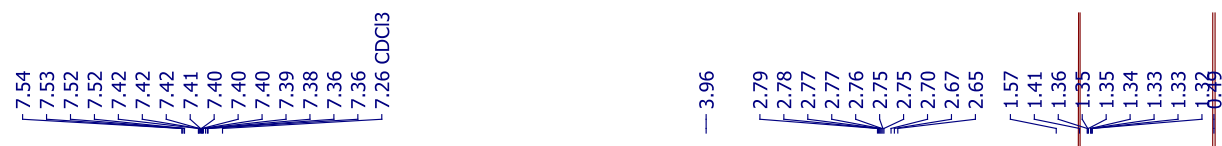<smiles>CC(C)(C)OC(=O)N1CCC(C(=O)Sc2ccccc2)CC1</smiles>

$2 \mathbf{t}$

$\left({ }^{1} \mathrm{H} \mathrm{NMR}, \mathrm{CDCl}_{3}, 500 \mathrm{MHz}\right)$

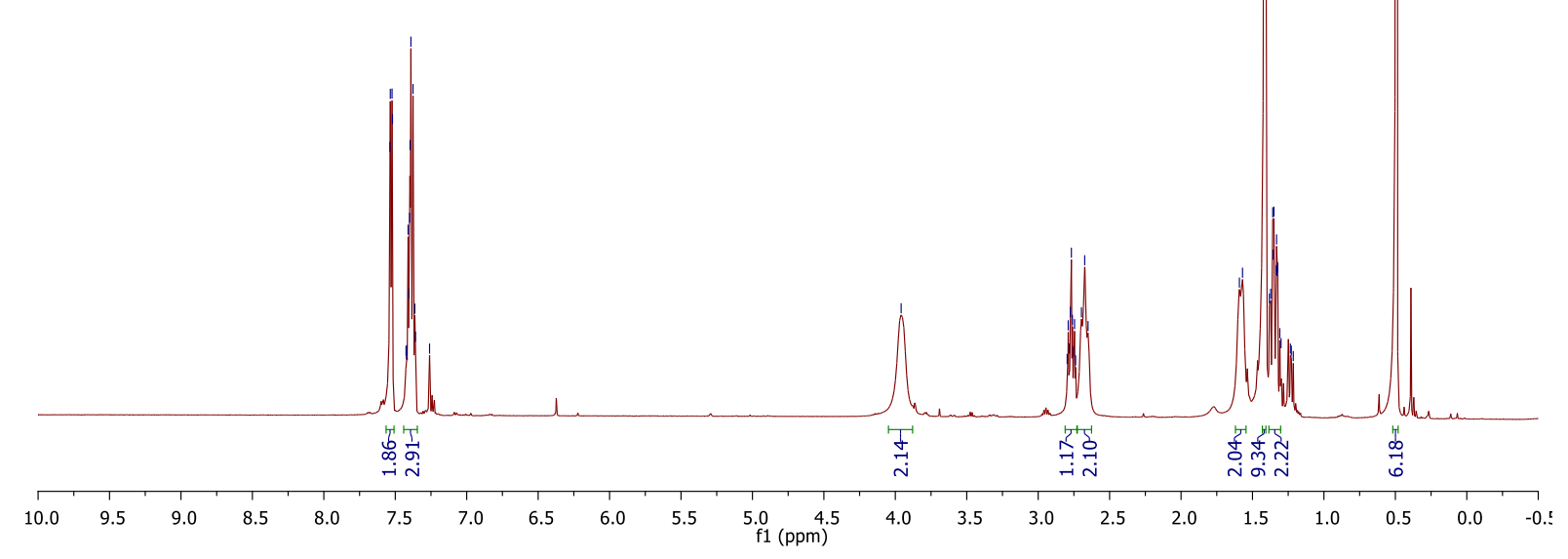

$$
\stackrel{i}{\stackrel{i}{0}}
$$$$
\text { ڤ̊n }
$$

$m$
$\mathrm{C}$
$\mathrm{O}$

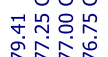

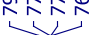
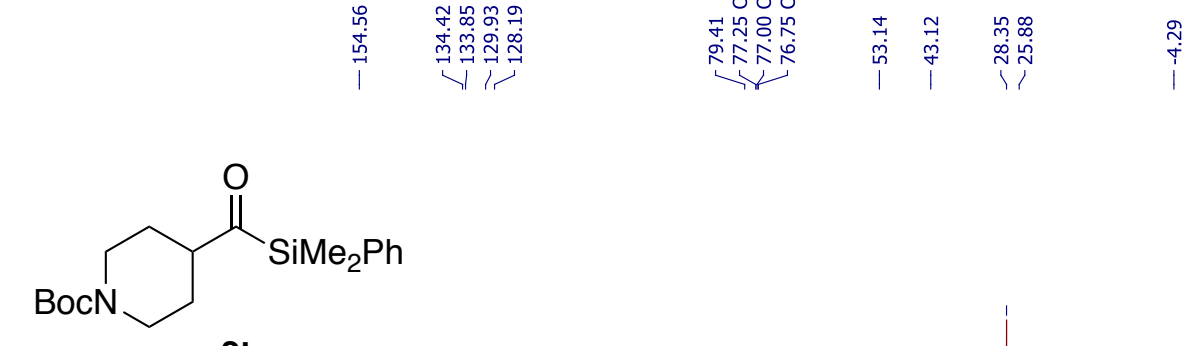

$2 t$

$\left({ }^{13} \mathrm{C} \mathrm{NMR}, \mathrm{CDCl}_{3}, 126 \mathrm{MHz}\right)$

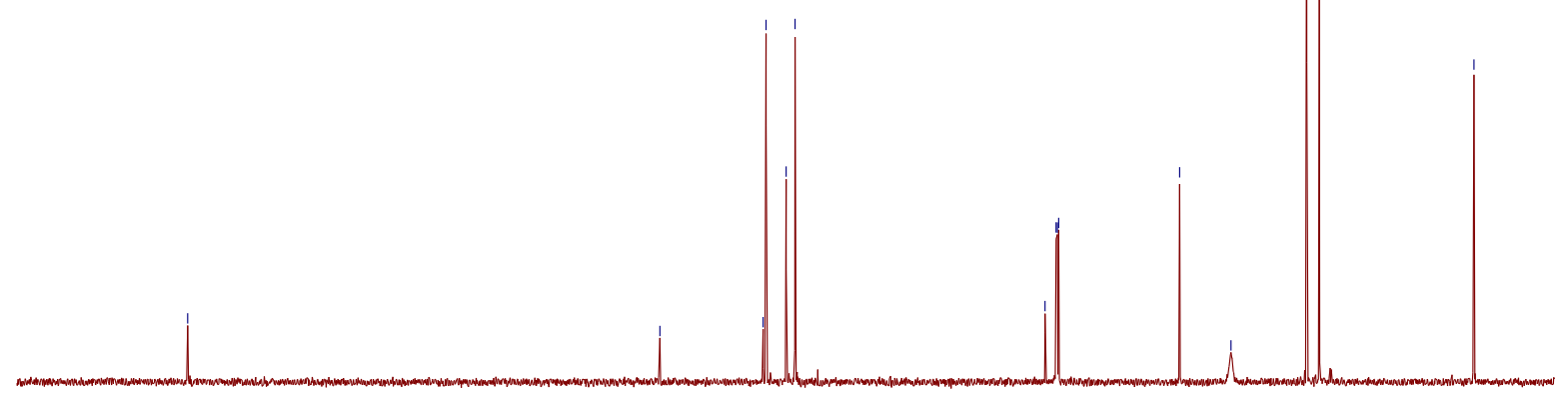

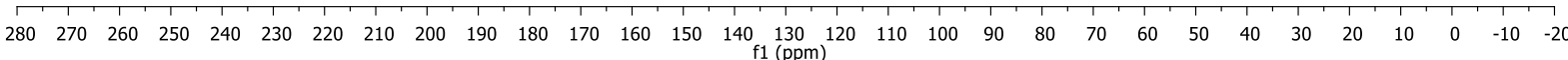




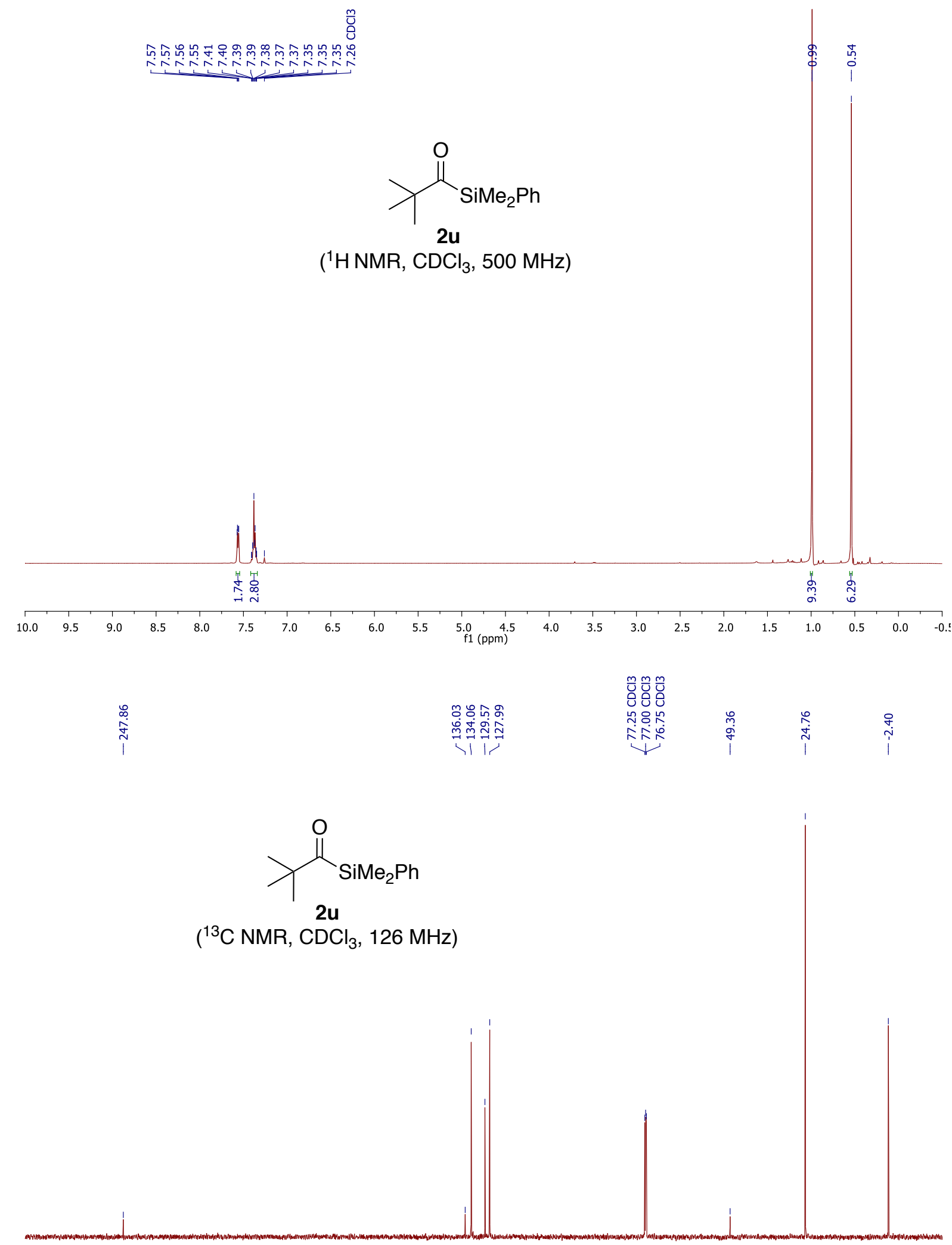

\begin{tabular}{rlllllllllllllllllllllllllllllllllllllllllllllll}
\hline 280 & 270 & 260 & 250 & 240 & 230 & 220 & 210 & 200 & 190 & 180 & 170 & 160 & 150 & 140 & 130 & 120 & 110 & 100 & 90 & 80 & 70 & 60 & 50 & 40 & 30 & 20 & 10 & 0 & -10 & -20
\end{tabular} 


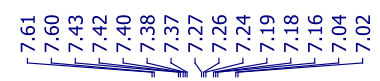

$\overbrace{\mathrm{SiMe}_{2} \mathrm{Ph}}^{\mathrm{O}}$

2v

$\left({ }^{1} \mathrm{H} \mathrm{NMR}, \mathrm{CDCl}_{3}, 500 \mathrm{MHz}\right)$
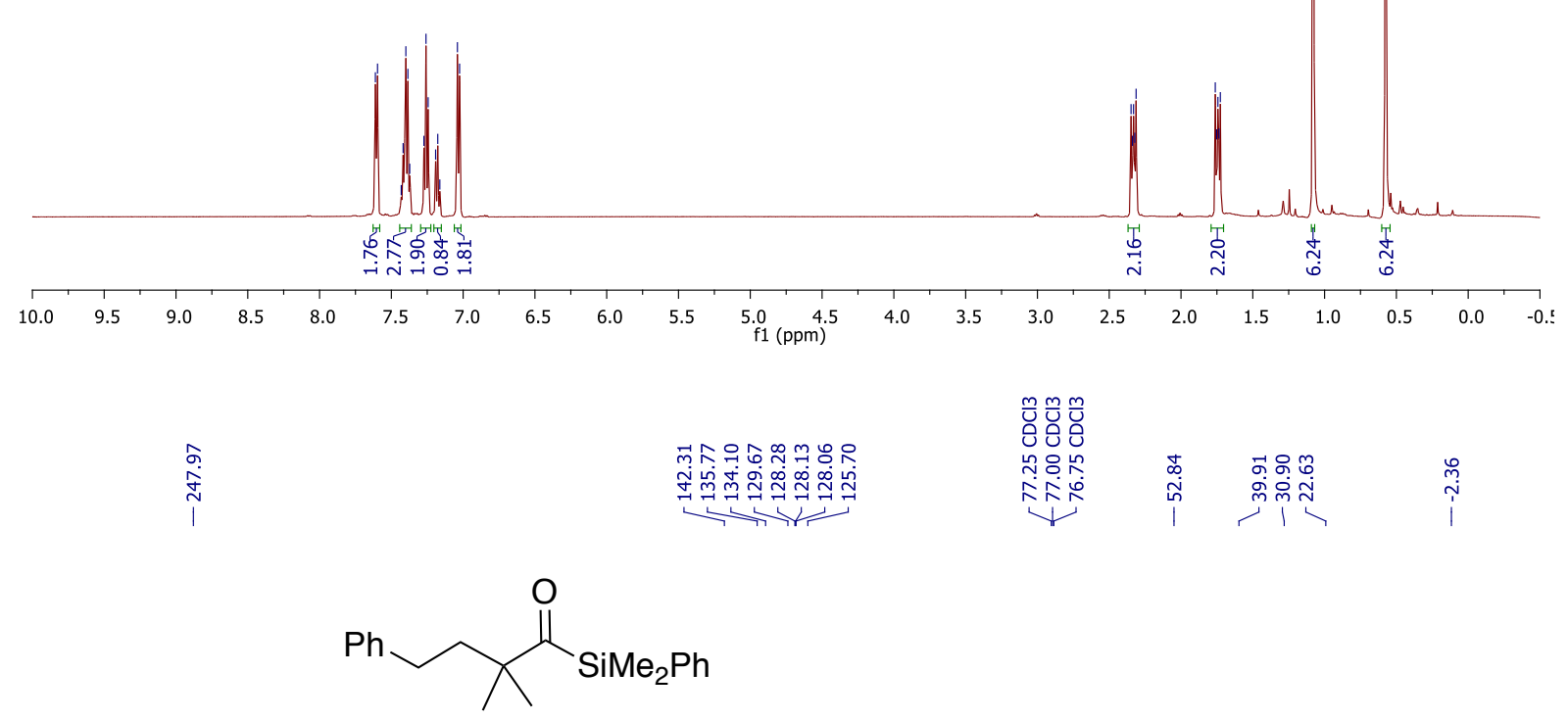

2v

$\left({ }^{13} \mathrm{C} \mathrm{NMR}, \mathrm{CDCl}_{3}, 126 \mathrm{MHz}\right)$

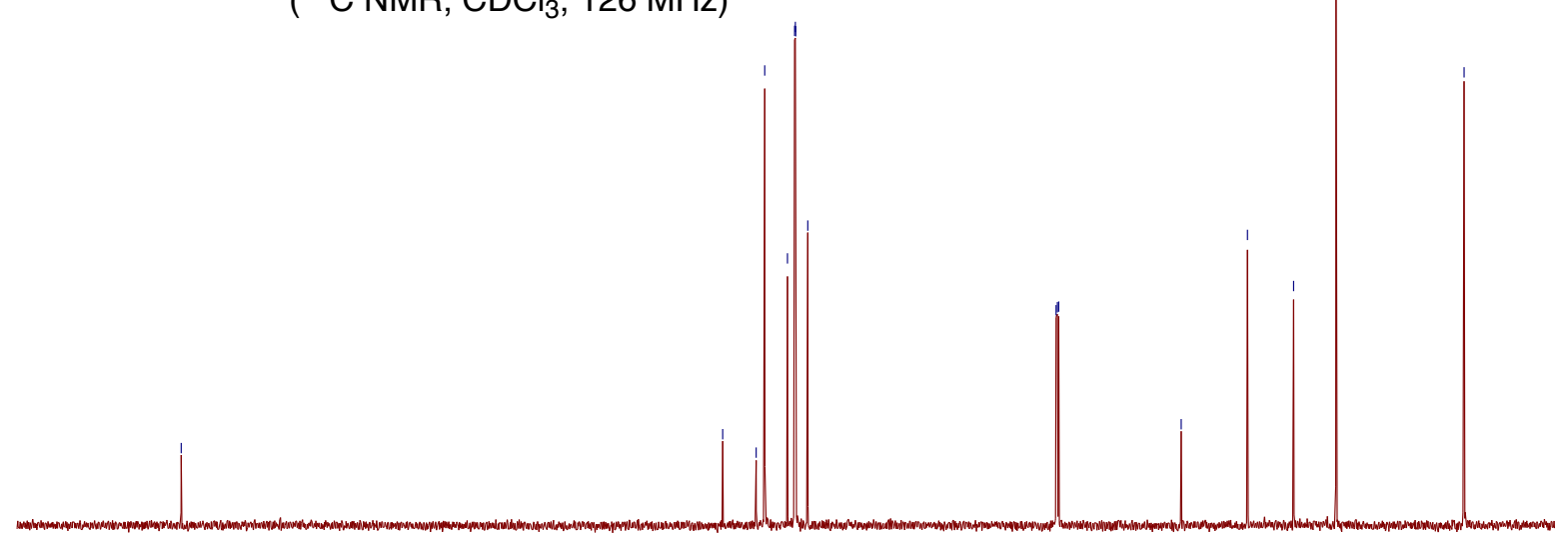

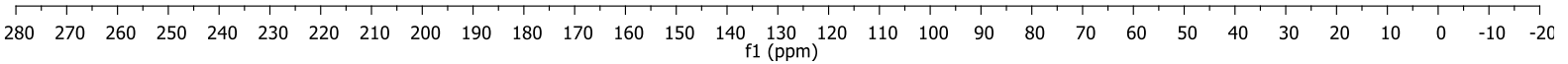


<smiles>CC(C)(Cc1ccccc1)C(=O)[AsH2-]c1ccccc1</smiles>

$2 w$

$\left({ }^{1} \mathrm{H} \mathrm{NMR}, \mathrm{CDCl}_{3}, 500 \mathrm{MHz}\right)$
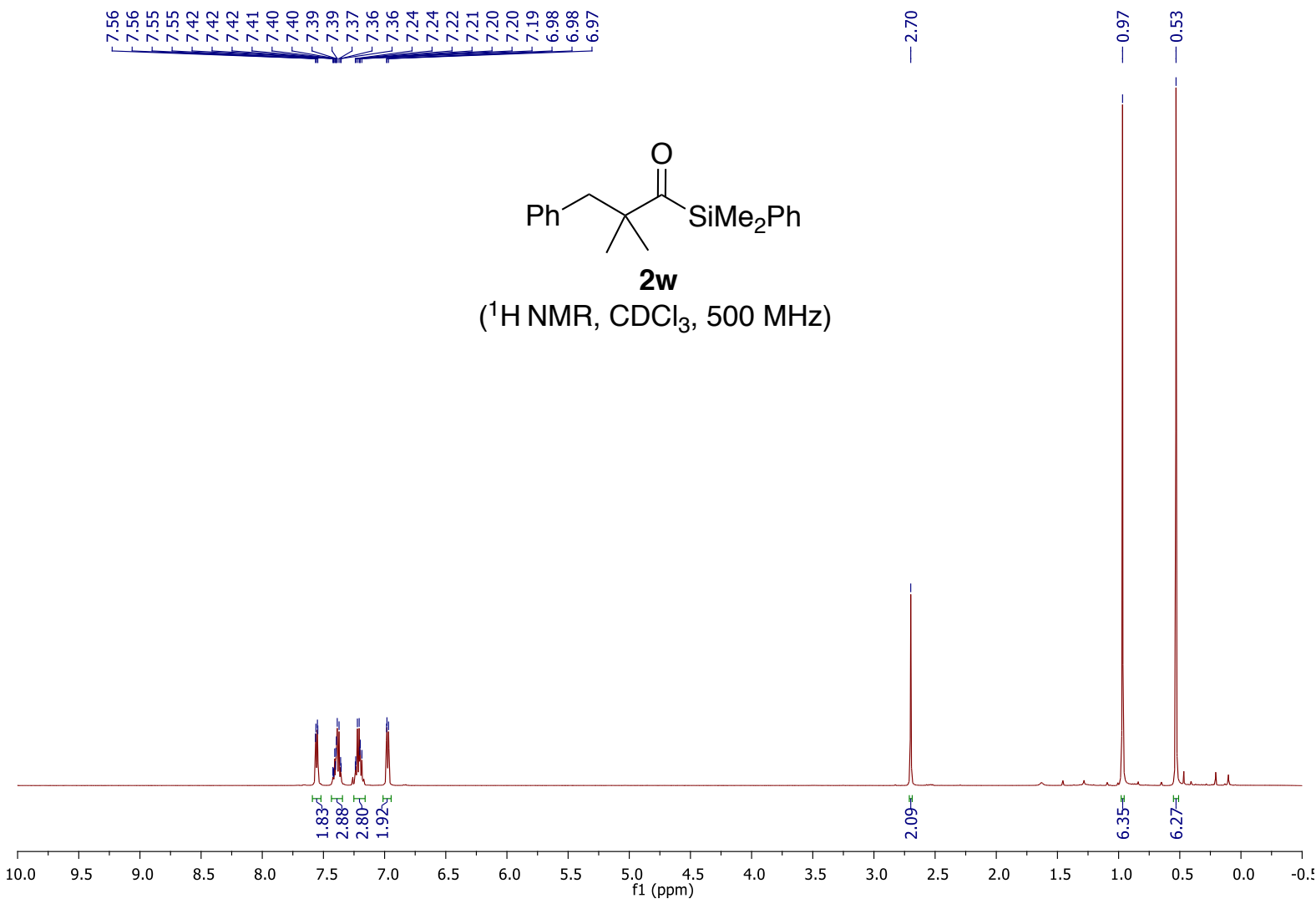

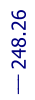

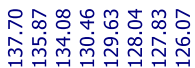

ํㅡㄴ

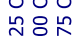

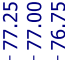

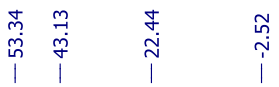<smiles>C[SiH](C(=O)C(C)(C)Cc1ccccc1)c1ccccc1</smiles>

2w

$\left({ }^{13} \mathrm{C} \mathrm{NMR}, \mathrm{CDCl}_{3}, 126 \mathrm{MHz}\right)$

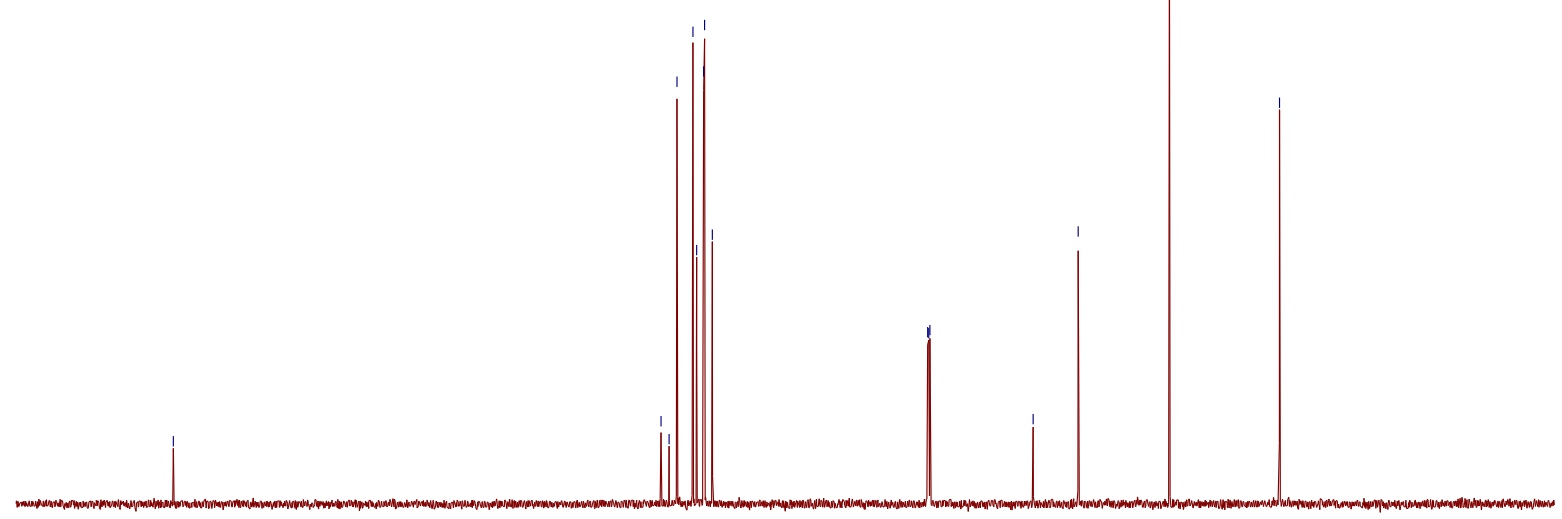

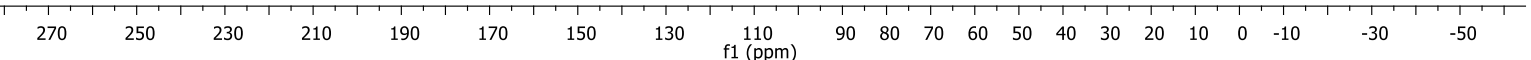




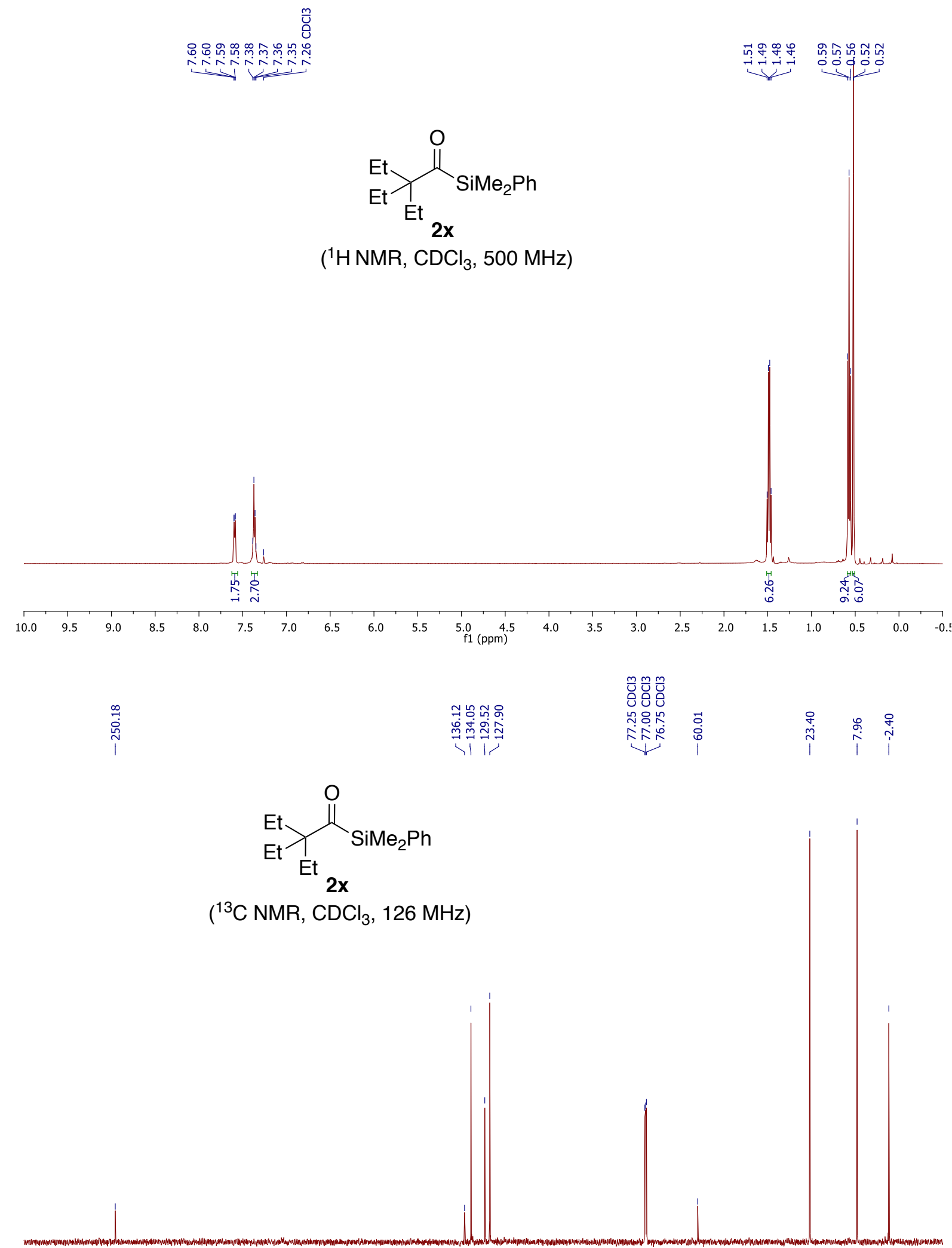

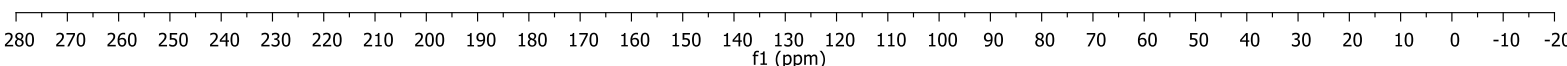



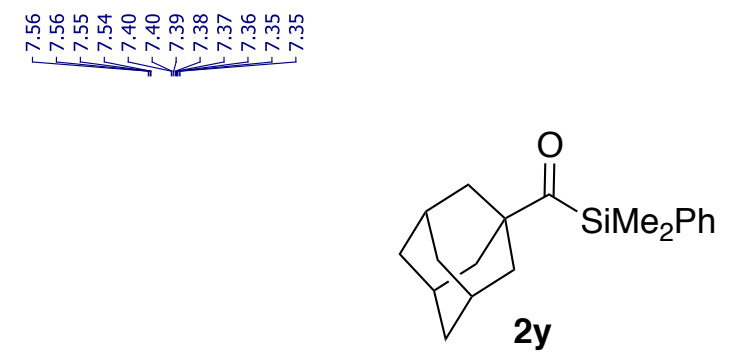

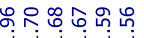

$\left({ }^{1} \mathrm{H} \mathrm{NMR}, \mathrm{CDCl}_{3}, 500 \mathrm{MHz}\right)$
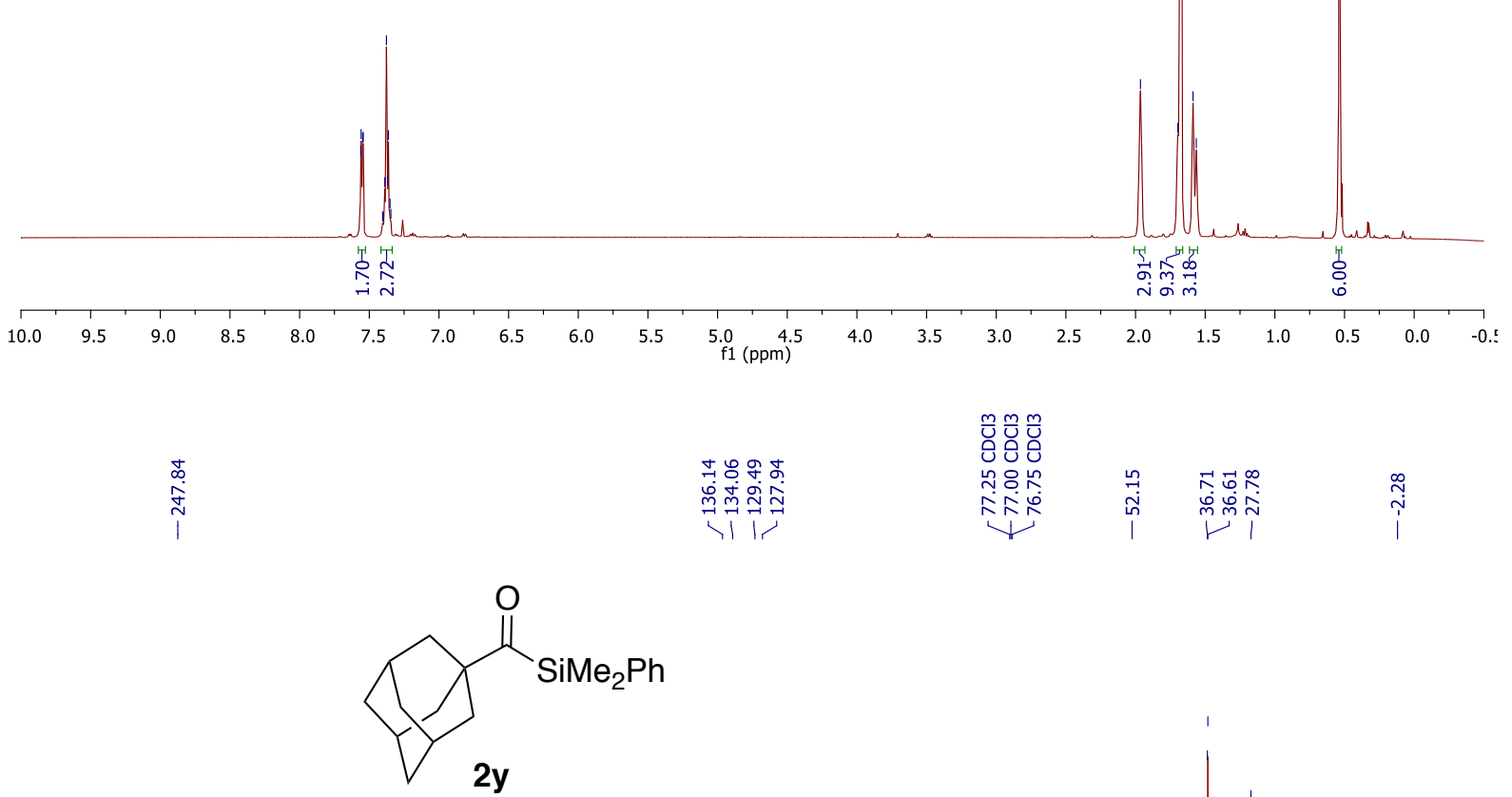

$\left({ }^{13} \mathrm{C} \mathrm{NMR}, \mathrm{CDCl}_{3}, 126 \mathrm{MHz}\right)$

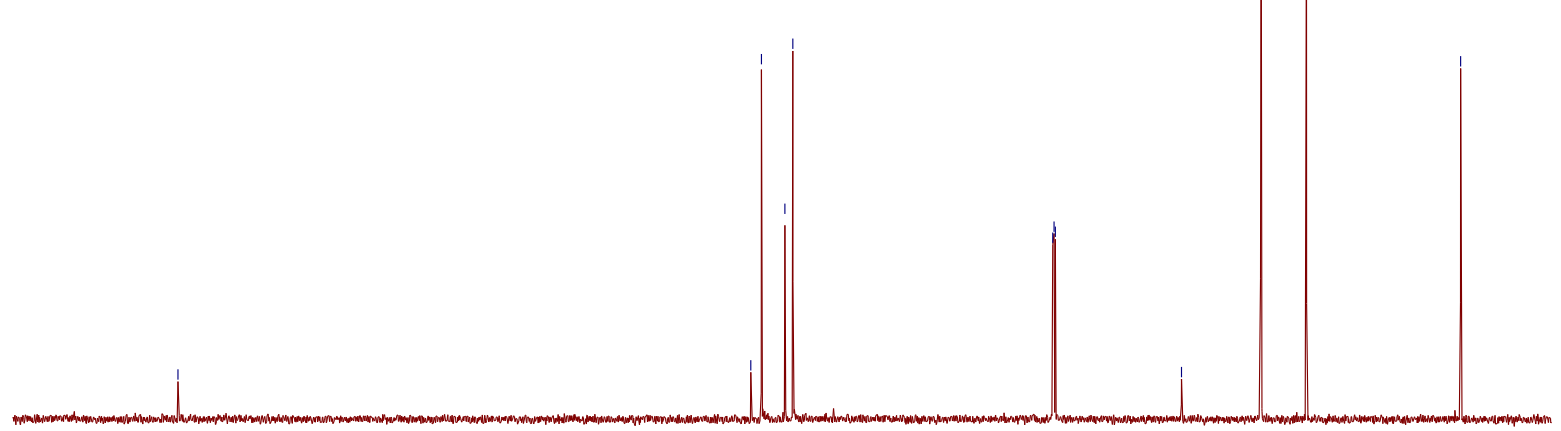

$\begin{array}{llllllllllllllllllllllllllllllllll}280 & 270 & 260 & 250 & 240 & 230 & 220 & 210 & 200 & 190 & 180 & 170 & 160 & 150 & 140 & 130 & 120 & 110 & 100 & 90 & 80 & 70 & 60 & 50 & 40 & 30 & 20 & 10 & 0 & -10 & -20\end{array}$ 


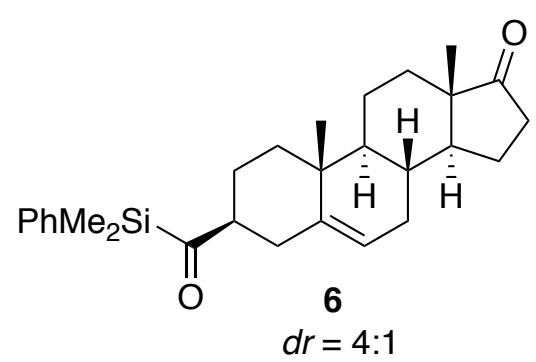

$\left({ }^{1} \mathrm{HNMR}, \mathrm{CDCl}_{3}, 500 \mathrm{MHz}\right)$
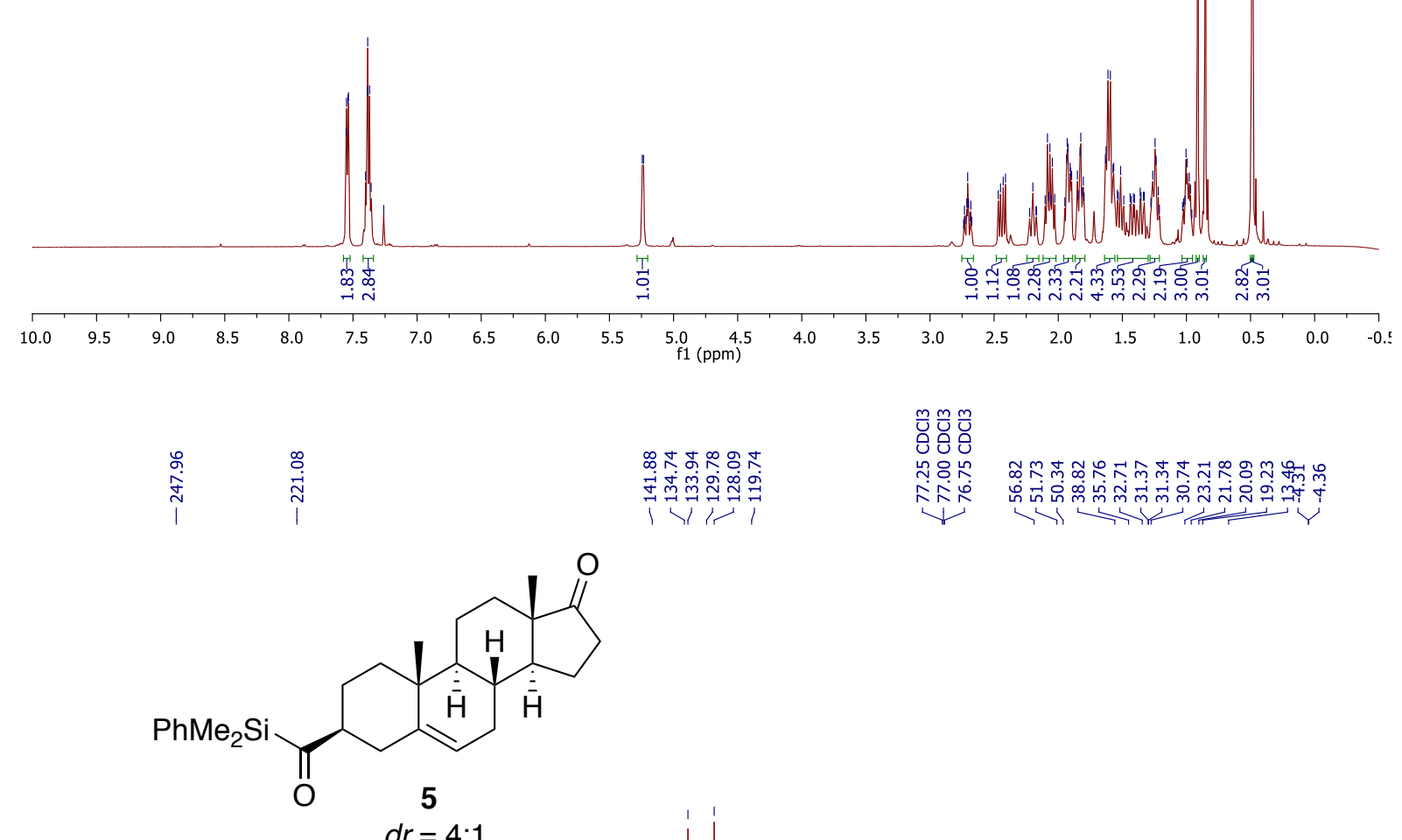

$\left({ }^{13} \mathrm{C} \mathrm{NMR}, \mathrm{CDCl}_{3}, 126 \mathrm{MHz}\right)$

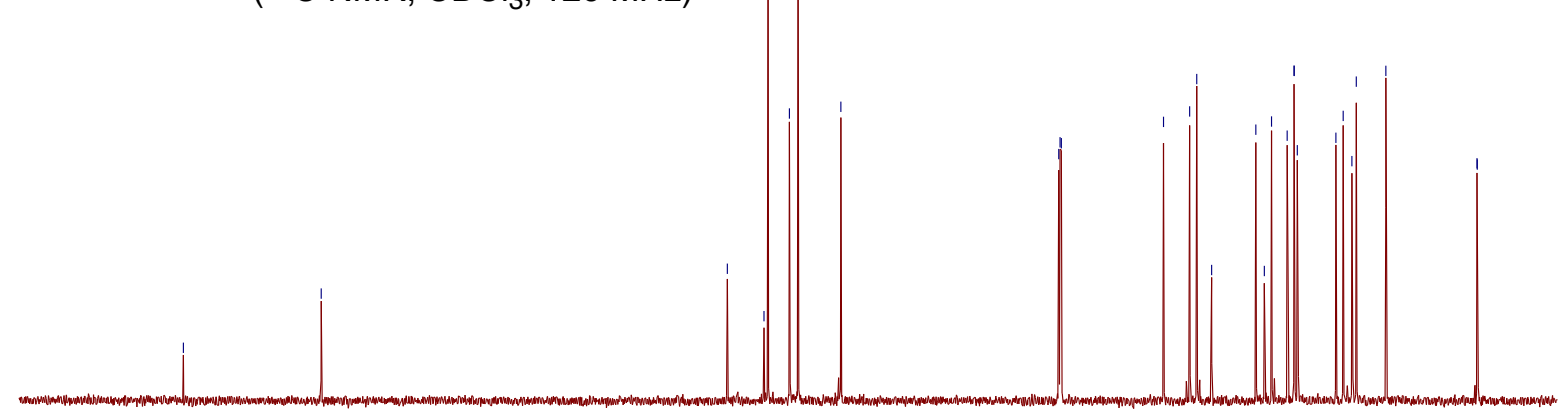

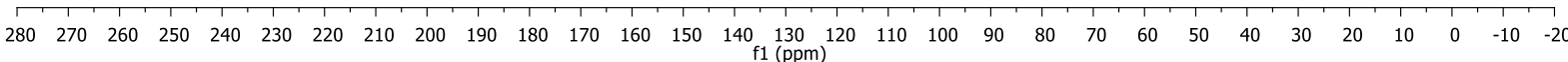




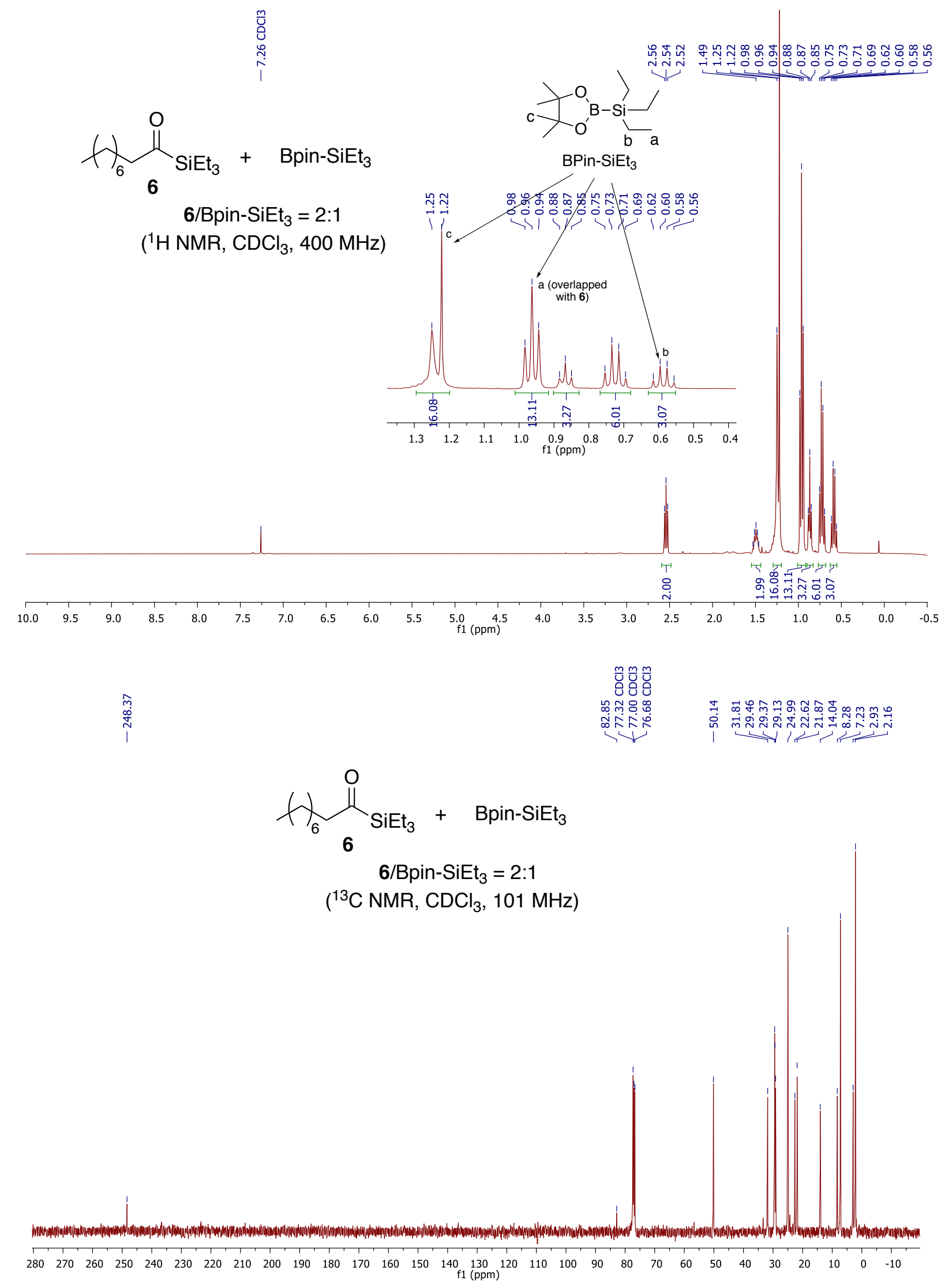



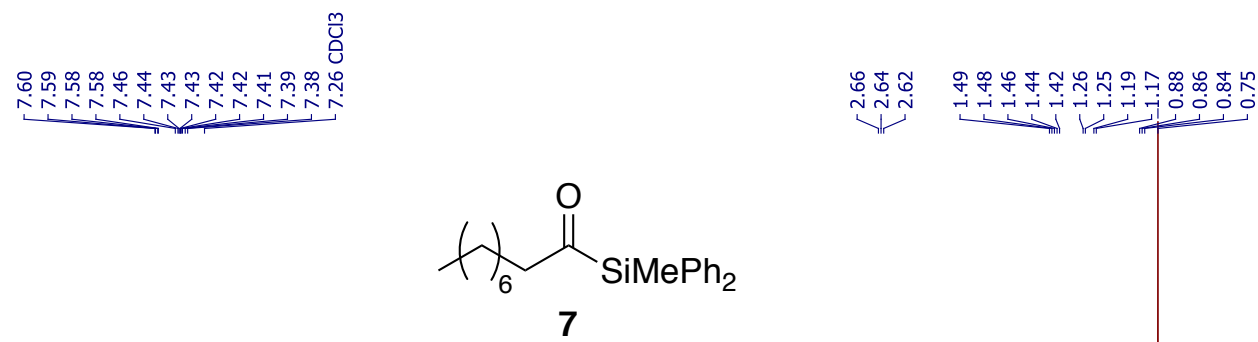

$\left({ }^{1} \mathrm{H} \mathrm{NMR}, \mathrm{CDCl}_{3}, 400 \mathrm{MHz}\right)$

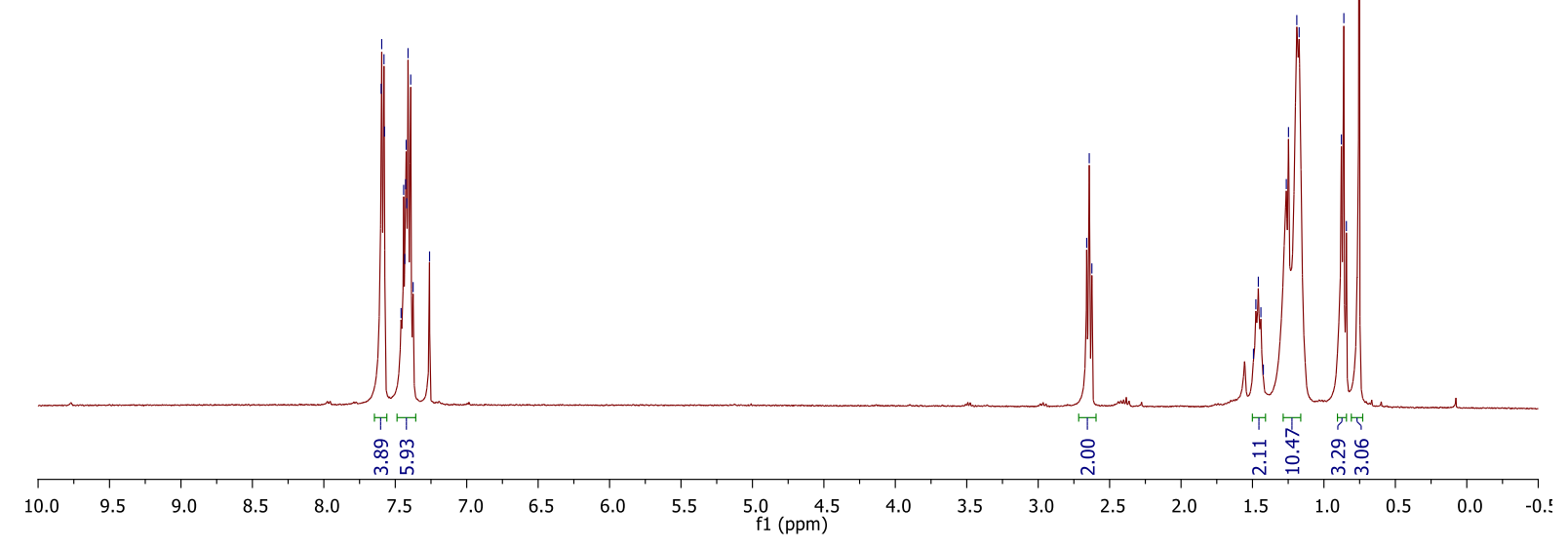

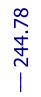
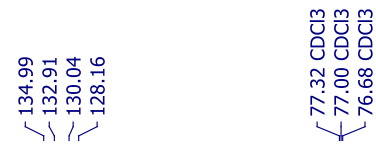

no

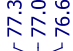

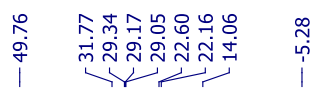

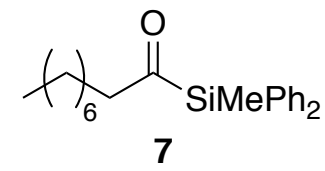

$\left({ }^{13} \mathrm{C} \mathrm{NMR}, \mathrm{CDCl}_{3}, 101 \mathrm{MHz}\right)$

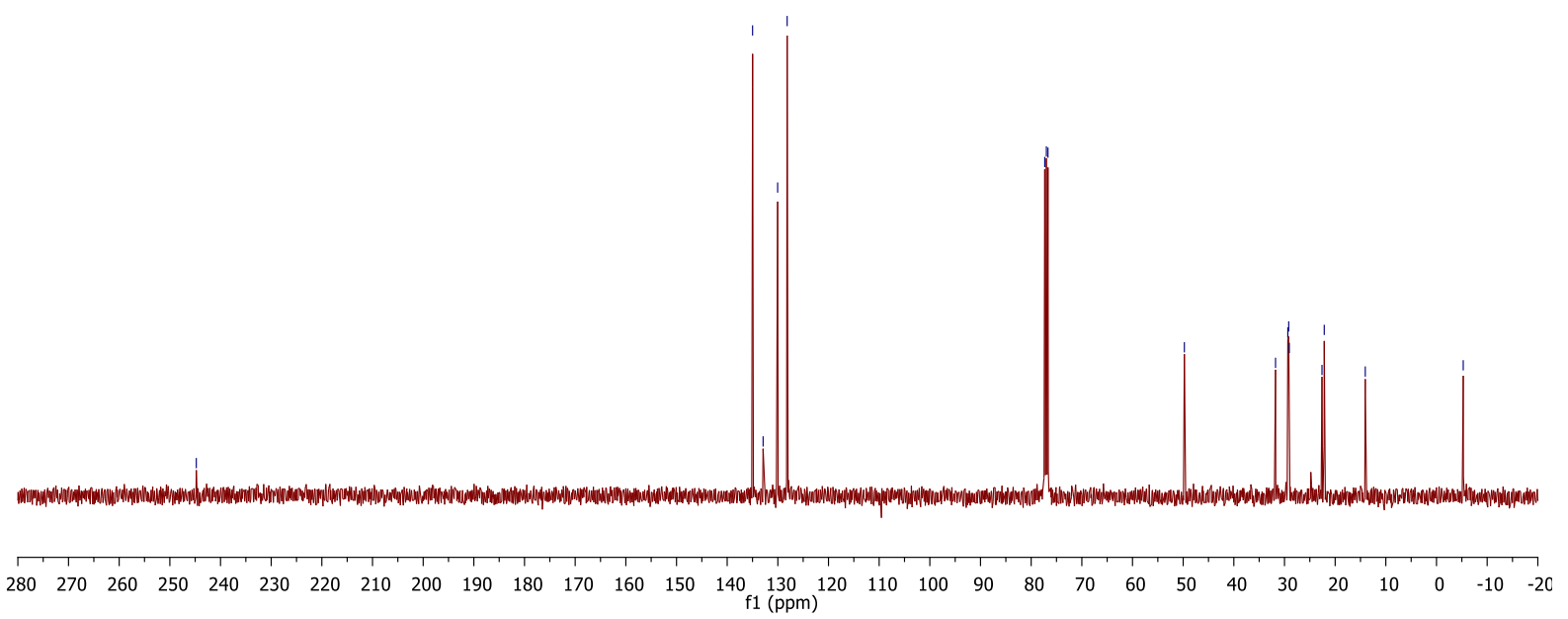



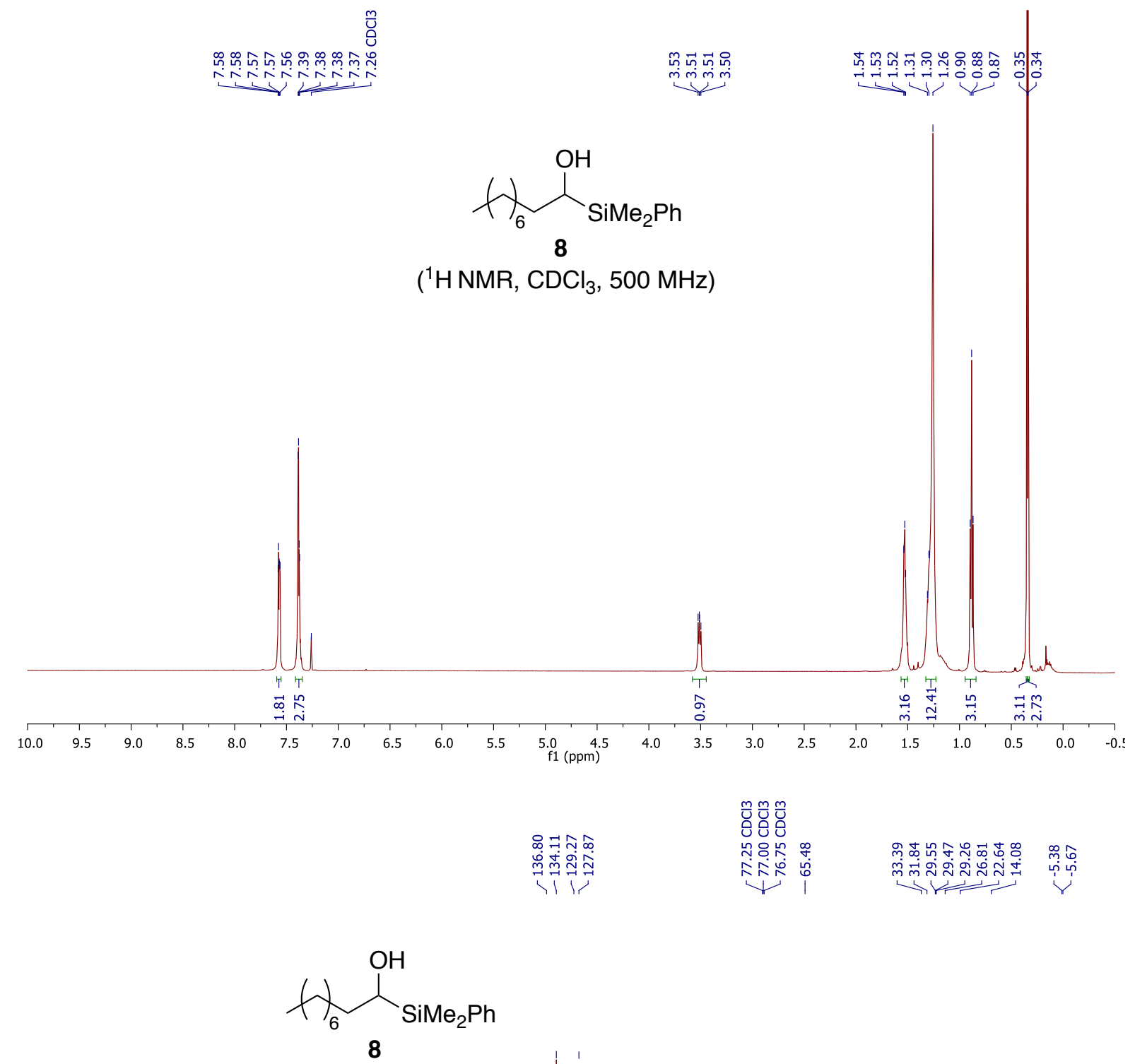

$\left({ }^{13} \mathrm{C} \mathrm{NMR}, \mathrm{CDCl}_{3}, 126 \mathrm{MHz}\right)$

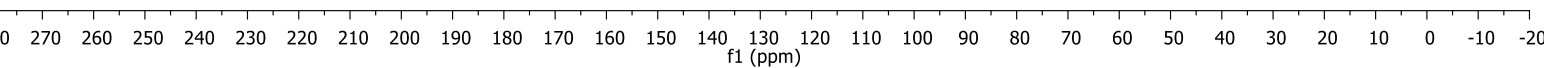



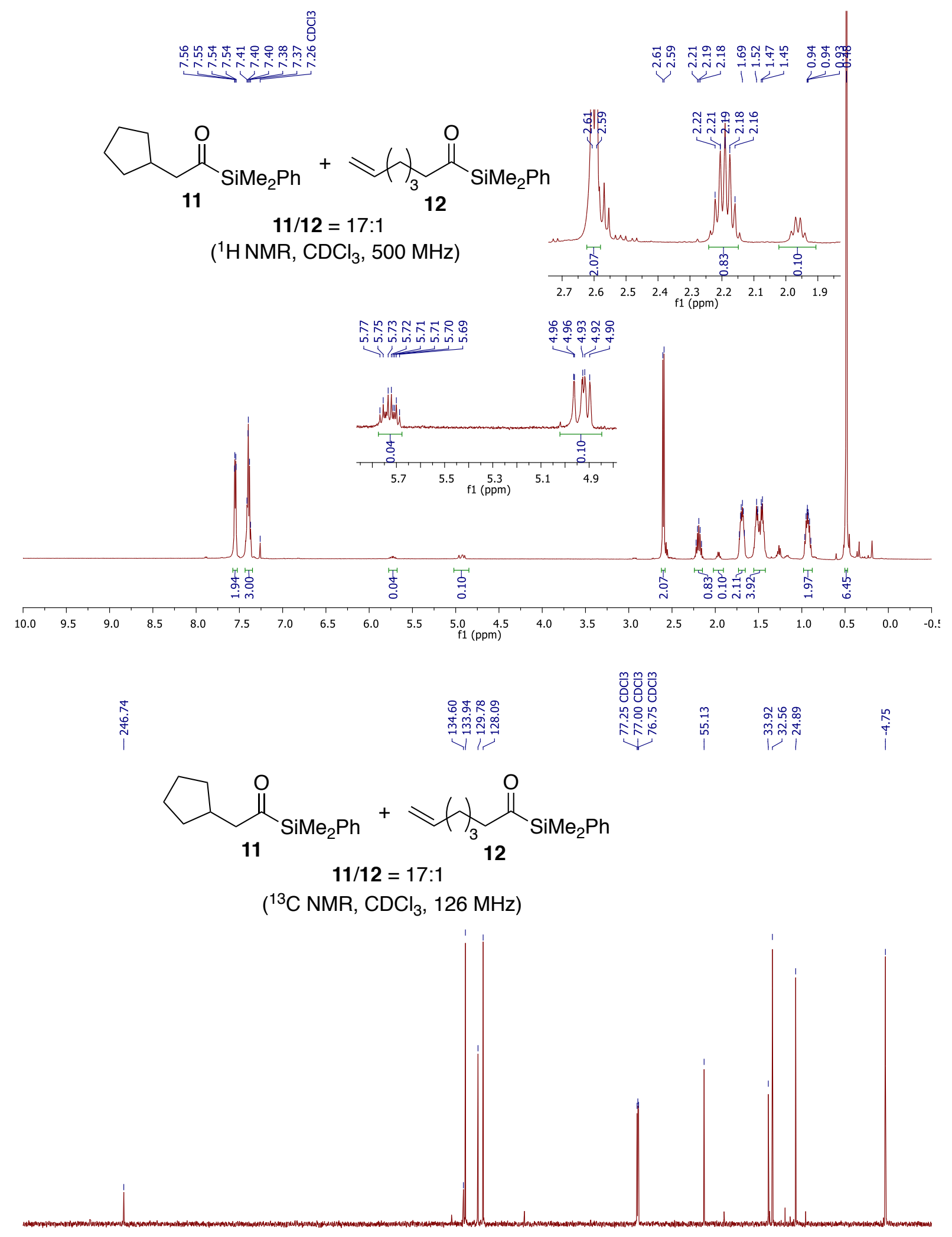

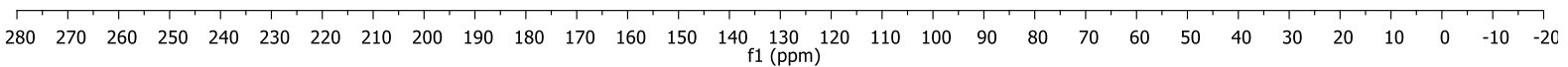




\section{References}

1 Xie, W.; Chang, S. Angew.Chem. Int. Ed. 2016, 55, 1876.

2 Santoro, O.; Collado, A.; Slawin, A. M. Z.; Nolan, S. P.; Cazin, C. S. J. Chem. Commun. 2013, 49, 10483.

${ }^{3}$ Semba, K.; Fujihara, T.; Xu, T.; Terao, J.; Tsuji, Y. Adv. Synth. Catal. 2012, 354, 1542.

${ }^{4}$ Boebel, T. A.; Hartwig, J. F. Organometallics 2008, 27, 6013.

${ }^{5}$ Suginome, M.; Matsuda, T.; Ito, Y. Organometallics 2000, 19, 4647.

6 Yadav, J. S.; Reddy, U. V. S.; Reddy, B. V. S. Tetrahedron Lett. 2009, 50, 5984.

7 Zhao, S; Mankad, N. P. Angew. Chem. Int. Ed. 2018, 57, 5867-5870.

${ }^{8}$ Dang, H.; Cox, N.; Lalic, G. Angew. Chem. Int. Ed. 2014, 53, 752.

9 Nguyen, J. D.; D’Amato, E. M.; Narayanam, J. M. R.; Stephenson, C. R. J. Nat. Chem. 2013, 4, 854.

10 Wang, Y.; Dong, X.; Larock, R. C. J. Org. Chem. 2003, 68, 3090.

11 Pye, D.; Cheng, L.-J.; Mankad, N. P. Chem. Sci. 2017, 8, 4750.

12 Someya, H.; Yorimitsu, H.; Oshima, K. Tetrahedron 2010, 66, 5993.

${ }^{13}$ Kyasa, S.; Meier, R. N.; Pardini, R. A.; Truttmann, T. K.; Kuwata, K. T.; Dussault, P. H. J. Org. Chem. 2015, 80, 12100.

14 Masada, H.; Murotani, Y. Bull. Chem. Soc. Jpn. 1980, 53, 1181.

15 Wu, X.; See, J. W. T.; Xu, K.; Hirao, H.; Roger, J.; Hierso, J.-C.; Zhou, J. Angew. Chem., Int. Ed. 2014, $53,13573$.

16 Cheng, L.-J.; Mankad, N. P.J. Am. Chem. Soc. 2017, 139, 10200.

17 Kleeberg, C.; Cheung, M. S.; Lin, Z.; Marder, T. B. J. Am. Chem. Soc. 2011, 133, 19060. 